Bulletin of the Seismological Society of America. Vol. 60, No. 6, pp. 1937-1987. December, 1970

\title{
SURFACE WAVES IN MULTILAYERED ELASTIC MEDIA. PART II. HIGHER MODE SPECTRA AND SPECTRAL RATIOS FROM POINT SOURCES IN PLANE LAYERED EARTH MODELS
}

\author{
By David G. Harkrider
}

\begin{abstract}
Phase and amplitude spectra of Rayleigh and Love waves are presented for two Earth models, one oceanic and one continental shield. The spectra of the first three Rayleigh modes and the first four Love modes are tabulated for point sources at selected depths. These tables along with computer algorithms described here allow one to estimate the amplitude spectra at nontabulated source depths.

The use of spectral ratios as a means of defermining source depth is investigated. A source depth of $20 \mathrm{~km}$ is obtained for the Fallon earthquake of July 201962. This depth agrees with previous estimates but the technique requires a fault-plane orientation which differs from radiation pattern solutions.
\end{abstract}

\section{INTRODUCTION}

Phase and amplitude spectra for the fundamental and the first two higher modes of Rayleigh waves and for the fundamental and the frst three higher modes of Love waves are presented in this paper at selected source or receiver depths for two Earth models, one oceanic and one continental shield.

The amplitudes are tabulated in a form which can be used to calculate the surfacewave spectra for any point source at that depth. The spectra for intermediate source depths can be interpolated from the tables using a simple computer program.

The theory and computer algorithms used in calculating the tables are given along with the long- and short-period asymptotes.

Theoretical surface-wave spectra have been useful in source mechanism studies involving amplitude equilization and radiation patterns. A potentially important technique is surface-wave spectral ratios. The advantages and disadvantages of using spectral ratios to determine source depth are discussed. The technique is then applied to the Fallon earthquake of July 201962.

THEORY

In the following, we will restrict ourselves to the far-field expressions for a point force, couple and double couple derived in Harkrider (1964) and Ben-Menahem and Harkrider (1964). The near-field expressions for surface waves due to a point force can be obtained from Harkrider (1964). The techniques of Ben-Menahem and Toksöz (1963), Haskell (1963), and Ben-Menahem and Harkrider (1964) can be applied to the point force solutions to obtain the far and near fields generated by higher order multipole sources.

The spectral far-field solutions for Love and Rayleigh waves may be written as

$$
U=S k^{m} e^{-i(1+2 m) \pi / 4} \chi(\theta, h) E \frac{e^{-i k r}}{r^{1 / 2}}
$$

where $U=$ spectral horizontal Rayleigh or Love surface displacement, $S=$ spectral 
source function, $m=0$, point force and $m=1$, couple or double couple, $k=\omega / c$ Rayleigh or Love angular wave number, $h=$ source depth, and $\theta=$ azimuth.

$$
\begin{aligned}
& E_{R}=\epsilon_{0} \underline{A}_{R} k_{R}^{-1 / 2} . \\
& E_{L}=\underline{A}_{L} k_{L}^{-1 / 2} .
\end{aligned}
$$

$\epsilon_{0}=-\left[\dot{u}_{0}^{*} / \dot{w}_{0}\right]$, Rayleigh-wave surface ellipticity, $\underline{A}=$ Rayleigh or Love amplitude response, $r=$ epicentral distance; and the subscripts $R$ and $L$ denote quantities associated with Rayleigh and Love waves, respectively.

TABLE 1

\begin{tabular}{|c|c|c|}
\hline \multirow{2}{*}{ Coefficient } & \multicolumn{2}{|c|}{ Point Force } \\
\hline & Love & Rayleigh \\
\hline$d_{0}$ & 0 & $\sin \lambda \sin \delta W(h)$ \\
\hline$d_{\mathbf{i}}$ & $\cos \lambda V(h)$ & $-\sin \lambda \cos \delta A(h)$ \\
\hline$d_{2}$ & $-\sin \lambda \cos \delta V(h)$ & $-\cos \lambda A(h)$ \\
\hline$d_{3}$ & 0 & 0 \\
\hline$d_{4}$ & 0 & 0 \\
\hline \multirow{2}{*}{ Coefficient } & \multicolumn{2}{|c|}{ Couple } \\
\hline & Love & Rayleigh \\
\hline$d_{0}$ & $-\frac{1}{2} \cos \lambda \sin \delta V(h)$ & $\frac{1}{4} \sin \lambda \sin 2 \delta B(h)$ \\
\hline$d_{1}$ & $\cos \lambda \cos \delta G(h)$ & $\sin \lambda\left[W(h)-\cos ^{2} \delta C(h)\right]$ \\
\hline$d_{2}$ & $-\sin \lambda \cos ^{2} \delta G(h)$ & $\cos \lambda \cos \delta[W(h)-C(h)]$ \\
\hline$d_{3}$ & $\frac{1}{4} \sin \lambda \sin 2 \delta V(h)$ & $\frac{1}{2} \cos \lambda \sin \delta A(h)$ \\
\hline$d_{4}$ & $\frac{1}{2} \cos \lambda \sin \delta V(h)$ & $-\frac{1}{4} \sin \lambda \sin 2 \delta A(h)$ \\
\hline \multirow{2}{*}{ Coefficient } & \multicolumn{2}{|c|}{ Double Couple } \\
\hline & Love & Rayleigh \\
\hline$d_{0}$ & 0 & $\frac{1}{2} \sin \lambda \sin 2 \delta B(h)$ \\
\hline$d_{1}$ & $\cos \lambda \cos \delta G(h)$ & $-\sin \lambda \cos 2 \delta C(h)$ \\
\hline$d_{2}$ & $-\sin \lambda \cos 2 \delta G(h)$ & $-\cos \lambda \cos \delta C(h)$ \\
\hline$d_{3}$ & $\frac{1}{2} \sin \lambda \sin 2 \delta V(h)$ & $\cos \lambda \sin \delta A(h)$ \\
\hline$d_{4}$ & $\cos \lambda \sin \delta V(h)$ & $-\frac{1}{2} \sin \lambda \sin 2 \delta A(h)$ \\
\hline
\end{tabular}

Radiation Pattern Coefficients

The complex radiation pattern function is

$$
\chi(\theta, h)=d_{0}+i\left(d_{1} \sin \theta+d_{2} \cos \theta\right)+d_{3} \sin 2 \theta+d_{4} \cos 2 \theta .
$$

The coefficients $d_{i}$ are given in Table 1 . The quantities $W(h), A(h), C(h), B(h), V(h)$ and $G(h)$ used in Table 1 are given in terms of the Thomson-Haskell displacementstress vector elements (Haskell, 1953) as

$$
\begin{aligned}
W(h) & =\left[\dot{w}_{S}(h) / \dot{w}_{0}\right] \\
A(h) & =-\left[\dot{u}_{S}{ }^{*}(h) / \dot{w}_{0}\right] \\
C(h) & =-\frac{1}{\mu_{S}}\left[\tau_{R S}(h) /\left(\dot{w}_{0} / C_{R}\right)\right]
\end{aligned}
$$




$$
\begin{aligned}
& B(h)=-\left\{\left(3-4 \frac{\beta_{S}{ }^{2}}{\alpha_{S}{ }^{2}}\right)\left[\dot{u}_{S}^{*}(h) / \dot{w}_{0}\right]+\frac{2}{\rho_{S} \alpha_{S}{ }^{2}}\left[\sigma_{R S}^{*}(h) /\left(\dot{w}_{0} / C_{R}\right)\right]\right\} \\
& V(h)=\left[\dot{v}_{S}(h) / \dot{v}_{0}\right]
\end{aligned}
$$

and

$$
G(h)=\frac{1}{\mu_{S}}\left[\tau_{L S}^{*}(h) /\left(\dot{v}_{0} / C_{L}\right)\right]
$$

The quantities in square brackets are the vector elements evaluated at source depth $h$. It should be noted that the preceeding expressions are different from the corresponding formula in Ben-Menahem and Harkrider (1964) in order to eliminate an artificial infinity in their $\chi(\theta)$ which resulted from zeros of $\left[\dot{w}_{S}(h) / \dot{w}_{0}\right]$ and $\left[\dot{v}_{s}(h) / \dot{v}_{0}\right]$. In addition their relations for $G(h)$ included a sign misprint.

In order to obtain expressions for higher order multipoles, it is necessary to differentiate the normalized displacement-stress quantities in square brackets with respect to source depth. These derivatives are

$$
\begin{aligned}
& \frac{\partial}{\partial h}\left[\frac{\dot{u}_{S}{ }^{*}(h)}{\dot{w}_{0}}\right]=k_{R}\left\{\left[\frac{\dot{w}_{S}(h)}{\dot{w}_{0}}\right]+\frac{1}{\mu_{S}}\left[\frac{\tau_{R S}(h)}{\dot{w}_{0} / C_{R}}\right]\right\} \\
& \frac{\partial}{\partial h}\left[\frac{\dot{w}_{S}(h)}{\dot{w}_{0}}\right]=-\frac{k_{R}}{\left(\lambda_{S}+2 \mu_{S}\right)}\left\{\lambda_{S}\left[\frac{\dot{u}_{S}^{*}(h)}{\dot{w}_{0}}\right]+\left[\frac{\sigma_{R S}^{*}(h)}{\dot{w}_{0} / C_{R}}\right]\right\} \\
& \frac{\partial}{\partial h}\left[\frac{\dot{v}_{S}(h)}{\dot{v}_{0}}\right]=-\frac{k_{L}}{\mu_{S}}\left[\frac{\tau_{L S}^{*}(h)}{\dot{v}_{0} / C_{L}}\right] \\
& \frac{\partial}{\partial h}\left[\frac{\sigma_{R S}^{*}(h)}{\dot{w}_{0} / C_{R}}\right]=k_{R}\left\{\rho_{S} C_{R}{ }^{2}\left[\frac{\dot{w}_{S}(h)}{\dot{w}_{0}}\right]+\left[\frac{\tau_{R S}(h)}{\dot{w}_{0} / C_{R}}\right]\right\} \\
& \frac{\partial}{\partial h}\left[\frac{\tau_{R S}(h)}{\dot{w}_{0} / C_{R}}\right]=-k_{R}\left\{\frac{\lambda_{S}}{\left(\lambda_{S}+2 \mu_{S}\right)}\left[\frac{\sigma_{R S}^{*}(h)}{\dot{w}_{0} / C_{R}}\right]\right. \\
& \left.+\left[\rho_{S} C_{R}{ }^{2}-\frac{4 \mu_{S}\left(\lambda_{S}+\mu_{S}\right)}{\left(\lambda_{S}+2 \mu_{S}\right)}\right]\left[\frac{\dot{u}_{S}^{*}(h)}{\dot{w}_{0}}\right]\right\}
\end{aligned}
$$

and

$$
\frac{\partial}{\partial h}\left[\frac{\tau_{L S}^{*}(h)}{\dot{v}_{0} / C_{L}}\right]=k_{L}\left(\rho_{S} C_{L}^{2}-\mu_{S}\right)\left[\frac{\dot{v}_{S}(h)}{\dot{v}_{0}}\right],
$$

where $\rho=$ density, $\mu=\rho \beta^{2}=$ rigidity, $\left(\lambda+2 \mu=\rho \alpha^{2}\right), \beta=$ shear-wave velocity, and $\alpha=$ compressional-wave velocity; the $s$ subscripts denotes quantities evaluated in the source layer. These relations were also used to obtain expressions for $A(h), B(h), C(h)$, and $G(h)$ resulting from differentiation of the point force solutions.

The amplitude response, $\underline{A}_{R}$ and $\underline{A}_{L}$ are functions of frequency, mode order, and the elastic properties of the multilayered array. They are independent of source type and 
depth and are defined in Harkrider (1964) as

and

$$
\underline{A}_{R}=\frac{G^{*} N-L^{*} H}{\left(\frac{\partial F_{R}}{\partial k}\right)_{\omega}}
$$

$$
\underline{A}_{L}=\frac{1}{\left[\frac{\dot{v}_{n-1}}{\dot{v}_{0}}\right]\left(\frac{\partial F_{L}}{\partial t}\right)_{\omega}}
$$

where $\left[\dot{v}_{n-1} / \dot{v}_{0}\right]$ is the normalized Love-wave displacement at the interface between the bottom layer and the terminating halfspace. The $F_{R}$ and $F_{L}$ are the Rayleigh- and Love-wave period equations, respectively.

The amplitude response factors can also be expressed in terms of depth integrals as

$$
\underline{A}_{R}=\left[2 C_{R} U_{R} \int_{0}^{\infty} \rho\left\{\left[\dot{u}^{*}(z) / \dot{w}_{0}\right]^{2}+\left[\dot{w}(z) / \dot{w}_{0}\right]^{2}\right\} d z\right]-1
$$

and

$$
\underline{A}_{L}=\left[2 C_{L} U_{L} \int_{0}^{\infty} \rho\left[\dot{v}(z) / \dot{v}_{0}\right]^{2} d z\right]^{-1}
$$

(Keilis-Borok and Yanovskaya, 1962; Harkrider and Anderson, 1966; Vlaar, 1966; Vilkovitch et al., 1966; and Saito, 1967 among others) where $U$ is the group velocity. Since $\rho$ is the density, the integrals are proportional to the kinetic energy densities of their respective surface waves.

The displacement-stress elements, the period equations, and the quantities, $G^{*}, N$, $L^{*}$ and $H$ can be expressed in terms of Thomson-Haskell matrices and their products. However, in calculating these terms for Rayleigh waves, the Thomson-Haskell technique results in numerical instabilities as frequency or mode order is increased. The following formulation, which is based on the Dunkin-Thrower algorithms, eliminates this numerical difficulty.

If we define the matrix operations

$$
R=T_{n}^{-1} A=T_{n}^{-1}\left(a_{n-1} \cdots a_{1}\right)
$$

where $A$ is the Rayleigh product matrix of the Thomson-Haskell layer matrices, $a_{m}$, for the $n-1$ solid layers and

$$
T_{n}^{-1}=\left[\begin{array}{cccc}
i \gamma_{n} r_{\alpha n}^{*} & \left(\gamma_{n}-1\right) & -\frac{i r_{\alpha n}^{*}}{\rho_{n} C^{2}} & \frac{1}{\rho_{n} C^{2}} \\
-\left(\gamma_{n}-1\right) & i \gamma_{n} r_{\beta n}^{*} & \frac{1}{\rho_{n} C^{2}} & \frac{i r_{\beta n}^{*}}{\rho_{n} C^{2}} \\
i \gamma_{n} r_{\alpha n}^{*} & -\left(\gamma_{n}-1\right) & -\frac{i r_{\alpha n}^{*}}{\rho_{n} C^{2}} & \frac{1}{\rho_{n} C^{2}} \\
\left(\gamma_{n}-1\right) & i \gamma_{n} \gamma_{\beta n}^{*} & -\frac{1}{\rho_{n} C^{2}} & \frac{i r_{\beta n}^{*}}{\rho_{n} C^{2}}
\end{array}\right]
$$


the scalars in equation (68) of Harkrider (1964) can be written as

$$
\begin{aligned}
L=R_{11} \equiv i R_{11}^{*}, K=R_{12}, G & =G_{13} \equiv i R_{13}^{*}, R=R_{14}, N=R_{21} \\
M & =R_{22} \equiv i R_{22}^{*}, H=R_{23} \text { and } S=R_{24} \equiv i R_{24}^{*} .
\end{aligned}
$$

Thus

$$
\begin{gathered}
R N-S L=-R\left(\begin{array}{l}
12 \\
14
\end{array}\right) \\
G N-H L=-R\left(\begin{array}{l}
12 \\
13
\end{array}\right) \\
G M-H K=-R\left(\begin{array}{l}
12 \\
23
\end{array}\right) \\
N K-L M=-R\left(\begin{array}{l}
12 \\
12
\end{array}\right)
\end{gathered}
$$

where

$$
R\left(\begin{array}{c}
j k \\
l m
\end{array}\right) \equiv R_{j l} R_{k m}-R_{j m} R_{k l}=-R\left(\begin{array}{c}
j k \\
m l
\end{array}\right)=R\left(\begin{array}{c}
k j \\
m l
\end{array}\right)
$$

The second compound matrices of $R$ are defined here as

$$
\mathfrak{R}_{s t}=R\left(\begin{array}{l}
j k \\
l m
\end{array}\right)
$$

where $S=1,2,3,4,5,6$, corresponds to pairs $j k=12,13,14,23,24,34$ with an identical correspondence of $t$ to $\mathrm{lm}$ (Gilbert and Backus 1966; and Thrower, 1965). In this notation, we have

$$
\begin{aligned}
& N K-L M=-\Re_{11} \\
& G N-H L=-\Re_{12} \equiv-i \Re_{12}^{*} \\
& R N-S L=-\Re_{13} \\
& G M-H K=-\Re_{14} .
\end{aligned}
$$

An important property of compound matrices is that if

$$
R=T_{n}^{-1} A
$$

then

$$
\mathcal{Q}={J_{n}}^{-1} \mathscr{Q}
$$


where $R, \Im_{n}^{-1}$ and $Q$ are the compound matrices of $R, T_{n}^{-1}$ and $A$, respectively. Thus

$$
\mathscr{Q}=a_{n-1} \cdots a
$$

where $a_{m}$ are the layer compound matrices given in Appendix I, and from equation (10) we have

$$
\begin{aligned}
&\left(J_{n}^{-1}\right)_{11}=\left(\gamma_{n}-1\right)^{2}-\gamma_{n}{ }^{2} \gamma_{\alpha n}^{*} r_{\beta n}^{*} \\
&\left(J_{n}^{-1}\right)_{12}=i\left(\rho_{n} c^{2}\right)^{-1} r_{\alpha n}^{*} \\
&\left(J_{n}{ }^{-1}\right)_{13}=\left(J_{n}^{-1}\right)_{14}=\left(\rho_{n} c^{2}\right)^{-1}\left[\left(\gamma_{n}-1\right)-\gamma_{n} r_{\alpha n}^{*} r_{\beta n}^{*}\right] \\
&\left(J_{n}^{-1}\right)_{15}=-i\left(\rho_{n} c^{2}\right)^{-1} r_{\beta n}^{*} \\
&\left(J_{n}^{-1}\right)_{16}=\left(\rho_{n} c^{2}\right)^{-2}\left(r_{\beta n}^{*} r_{\alpha n}^{*}-1\right)
\end{aligned}
$$

which are used to calculate $\mathfrak{R}_{11}, \mathfrak{R}_{12}, \mathfrak{R}_{13}$ and $\mathfrak{R}_{14}$.

The period equation for Rayleigh waves in a solid array is given by

$$
F_{R} \equiv-\Re_{11}=0 .
$$

It was noted by Rosenbaum (1964) and Dunkin (1965) that the coefficient of the dominant term in $\mathbb{R}_{11}$ at moderate to high frequencies was identically zero for all frequencies. Thus calculating $R_{11}$ from its Thomson-Haskell elements causes a loss of significant figures at these frequencies. Dunkin (1965) and Thrower (1965) pointed out that calculating $\mathbb{R}$ by means of the compound matrices eliminates this loss of significance at high frequencies.

In terms of the compound matrices, the ratio of horizontal to vertical displacement or velocity, i.e., surface ellipticity, is

$$
\left[\frac{\dot{u}_{0}}{\dot{w}_{0}}\right]=-\frac{R N-S L}{G N-H L}=-\frac{\Re_{13}}{R_{12}}=-\frac{\Re_{14}}{\Omega_{12}}
$$

since

$$
R N-S L \equiv G M-H K=-\Re_{14}
$$

This relation holds even if there is a liquid layer at the surface where $\left[\dot{u}_{0} / \dot{w}_{0}\right]$ is understood to be the ratio at the top of the solid array.

For the surface liquid layer, the period equation takes the form

$$
F_{R}=-\mathfrak{R}_{11}+\mathfrak{R}_{12} T^{*}
$$

where

$$
T=\left[\frac{\sigma_{0}}{\dot{w_{0}} / C}\right]=i T^{*}=\frac{i_{\rho_{0}} C^{2}}{r_{\alpha 0}} \tan \left(k r_{\alpha 0} d_{0}\right)
$$

and the 0 subscript denotes the physical parameters of the liquid surface layer. 
Defining $B^{m}$ as

$$
\begin{aligned}
B^{m} & =R A_{m-1}^{-1} \\
& =T_{n}^{-1} \mathrm{a}_{n-1} \cdots \mathrm{a}_{m} \text { where } A_{m}=\mathrm{a}_{m} \cdots \mathrm{a}_{1},
\end{aligned}
$$

we have that

$$
B^{m}=B^{m+1} a_{m}
$$

with

$$
B^{n}=R A_{n-1}^{-1} \equiv R A^{-1}=T_{n}^{-1}
$$

Making use of these relations the normalized displacement stress vector $U_{m}(z)$ given by

$$
U_{m}(z)=\left[\begin{array}{c}
\frac{\dot{u}_{m}(z)}{\dot{w}_{0}} \\
\frac{\dot{w}_{m}(z)}{\dot{w}_{0}} \\
\frac{\sigma_{m}(z)}{\dot{w}_{0} / C} \\
\frac{\tau_{R m}(z)}{\dot{w}_{0} / C}
\end{array}\right] \quad \text { for } \quad Z_{m-1} \leqq Z \leqq Z_{m}
$$

can be evaluated from the compound matrices by

$$
\left[U_{m}(z)\right]_{k}=\frac{B_{1 l}^{m}}{R_{11}} A_{m}\left({ }_{12}^{l k}\right)+T \frac{B_{1 l}^{m}}{R_{11}} A_{m}\left({ }_{13}^{l k}\right)
$$

where $T$ is zero for models without a surface liquid.

Using these relations gives very good results in calculating the fundamental and higher mode spectra at periods down to even a second for realistic earth models.

The source factor, $S$, in equation (1) is the same for Rayleigh and Love waves for nonpropagating point sources. For propagating sources, this factor will be a function of source dimensions, propagation velocity, and source time history. In general it will differ by terms involving the phase velocity for Rayleigh and Love waves. Representations of $S$ for various deterministic source models can be found in Ben-Menahem (1961), Ben-Menahem and Toksöz (1962), and Haskell (1964). Representations of some "statistical" source models can be found in Haskell (1966) and Aki (1967).

Even for the more complicated models, where $S$ and $\chi(\theta, h)$ cannot be readily separated into factors, the far-field solutions will contain combinations of the displacement-stress quantities or their integrals over the source volume.

\section{Numerical Techniques}

The Rayleigh- and Love-wave calculations presented in this paper were obtained using two programs written in FORTRAN IV for each type of surface wave. The first programs for both Rayleigh and Love waves calculate the dispersion and depth inde- 
pendent quantities for a given input period. In addition, the displacement-stress values are calculated for the mid-point depth of each layer. The output from these programs are then used as input to the source depth programs which evaluate the displacementstress quantities at any number of specified depths.

The flow for the dispersion programs is the same as that described in Press et al. (1961) and Harkrider and Anderson (1962) with the exception that the dispersion is calculated for a specified period instead of phase velocity. Thus the programs calculated the roots, $k_{j}$ of $F_{R}$ or $F_{L}=0$ for an input period. The Love-wave program uses the Thomson-Haskell formulation of $F_{L}$ and the Rayleigh-wave program uses the compound layer matrix formulation of $F_{R}$.

The compound layer matrices for Rayleigh waves are $(6 \times 6)$. The matrix multiplications in equation (17), which form the compound layer product matrix, appear to require a sequence of $(6 \times 6)$ matrix multiplications. Actually the largest matrix resulting from any multiplication in the program is a $(6 \times 3)$.

The Rayleigh period equation (19) for the solid array and (22) for a liquid surface layer use only the first column and the first two columns of the compound layer product matrix respectively. This considerably reduces the number of multiplications during the time-consuming root-hunting procedure since each $(6 \times 6)$ layer compound matrix can then multiply the previous $(6 \times 1)$ or $(6 \times 2)$ compound layer product matrix to obtain the next $(6 \times 1)$ or $(6 \times 2)$ compound layer product matrix as indicated by $a$ right to left multiplication of compound matrices in equation (17).

Once a root is found to the desired accuracy, the group velocity, $U$, the kinetic energy density, and the amplitude responses are calculated in the Rayleigh and the Love programs. In addition, the Rayleigh program uses the second and third columns of the compound product matrix in equation (20) to obtain the surface ellipticity.

The group velocity and the amplitude response are calculated by two different numerical techniques in both programs. In one technique, $U$ and $(\partial F / \partial k)_{\omega}$ are formed by computing the analytic derivatives with respect to $\omega$ and $k$ of the individual layer matrices and then using the chain rules. The second technique forms $A$ and $U$ by analytically computing the depth integrals in equation (8) and in similar equations for the group velocity (Harkrider and Anderson, 1966).

The Rayleigh-wave displacement-stress values at each interface are calculated by means of equation (27). Only the first row of $B^{m}$ is used, thus, $B^{m}$ as a $(1 \times 4)$ matrix is calculated and stored for each interface using equations (25). The multiplication of each $(1 \times 4)$ by a $(4 \times 4)$ to yield the desired $(1 \times 4)$ matrices starts at the half-space and proceeds to the surface layer. The first two columns of the compound product matrix of layers from the surface to the desired interface is combined with $B^{m}$ to yield $\left[U_{m}(z)\right]_{k}$. The dispersion programs then use the Thomson-Haskell matrix to evaluate $\left[U_{m}(z)\right]_{k}$ at the layer mid points. The Love-wave dispersion program used the Thomson-Haskell formulation for the entire displacement-stress calculation.

This same process is used for the source depth programs in which period, phase velocity, and ellipticity for Rayleigh waves are used as input. In these programs the Thomson-Haskell matrices continue the displacement-stress vectors from an interface to any specified depth in the adjacent layer.

In order to determine the significance of computed quantities, we try to use at least two theoretically equivalent but numerically different calculations. For the group velocity and the amplitude response this is done by using both the partial derivative and energy integral formulations. It is also possible to compare the very long-and short-period values with their asymptotic limits. 
The asymptotic limits of the amplitude response can be obtained easily from the Thomson-Haskell formulation and are given in Appendix II and Appendix III.

\section{Numerical Results}

Tables of quantities which can be used to calculate the surface-wave spectra for multipole point sources at depth are presented here for two extreme models of the Earth's crust and mantle structure - one oceanic and one continental shield (Figure 1). The layer thickness, $D$ in $\mathrm{km}$, compressional velocity, $\alpha$ in $\mathrm{km} / \mathrm{sec}$, shear velocity, $\beta$ in $\mathrm{km} / \mathrm{sec}$, and density, $\rho$ in $\mathrm{gm} / \mathrm{cm}^{3}$, are given in Table 2 for the two models.

The tables include the fundamental and first two higher modes of Rayleigh waves and the fundamental and first three higher modes of Love waves for the two models. The period in seconds, the phase velocity, $C$ in $\mathrm{km} / \mathrm{sec}$, the group velocity, $U$ in $\mathrm{km} / \mathrm{sec}$ and the spectral amplitude response, amplitude in $10^{-11}$ microns/dyne are given in

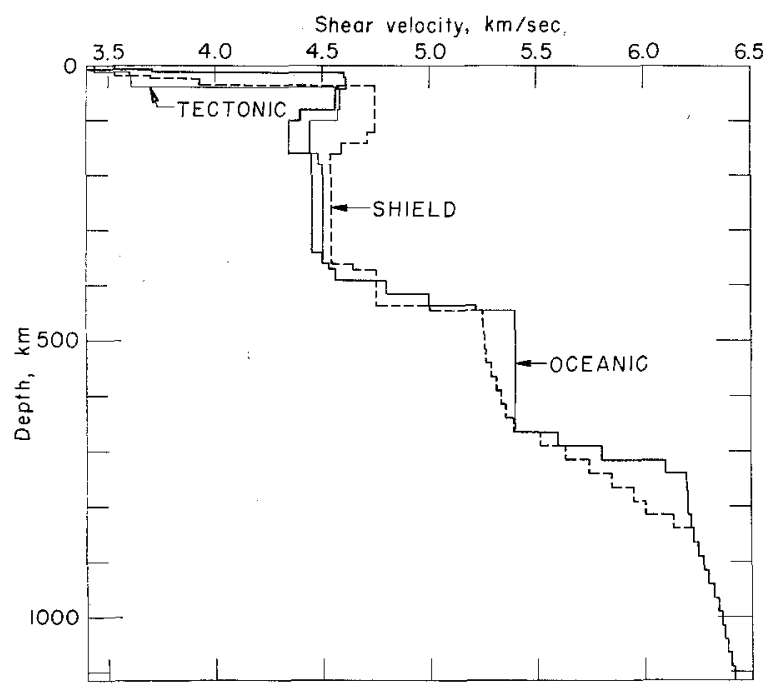

FIG. 1. Shear velocity as a function of depth for oceanic, tectonic, and shield earth models (Anderson and Toksöz, 1966; Anderson, 1966).

Tables 3 and 4 for the oceanic and shield models, respectively, and in Figures 2 to 7. The amplitude response multiplied by $k^{-1 / 2}$ to form the relative excitation in $10^{-12.5}$ $\mathrm{cm}^{3 / 2} /$ dyne is shown in Figures 6 and 7 .

The negative of the Rayleigh-wave surface ellipticity, $\left[\dot{u}_{0}^{*} / \dot{w}_{0}\right]$, is tabulated under the label of $U O / W O$ in Table 5.

The depth-dependent terms in $W(h), A(h), C(h), B(h), V(h)$ and $G(h)$ which determine $\chi(\theta, h)$ are tabulated in Tables 6 to 27 for 11 depths in each model. The source depths are $10,20,35,50,70,100,150,200,350,500$, and $700 \mathrm{~km}$. The quantities

$\left[\dot{u}_{S} *(h) / \dot{w}_{0}\right],\left[\dot{w}_{S}(h) / \dot{w}_{0}\right],\left[\sigma_{R S}(h) /\left(\dot{w}_{0} / C_{R}\right)\right],\left[\tau_{R S}(h) /\left(\dot{w}_{0} / C_{R}\right)\right]$,

$$
\left[\dot{v}_{S}(h) / v_{0}\right] \text { and }\left[\tau_{L S}(h) /\left(\dot{v}_{0} / C_{L}\right)\right]
$$

are given in these depth tables under columns labeled respectively $U M / W O, W M / W O$, $Z M / W O / K, X M / W O / K, V M / V O$, and $Y M / V O / K$.

Since these quantities are the elements of the Thomson-Haskell displacement-stress 
TABLE 2

Elastic-layer Parameters for the Oceanic and Shield Models OCEAN

\begin{tabular}{|c|c|c|c|c|c|c|}
\hline D & ALPHA & BEIA & RHO & MU & LAMOA & DEPTH \\
\hline 5.00 & 1.5200 & 0. & 1.0300 & 0.100000 & 2.379712 & 2.50 \\
\hline $\begin{array}{l}5.00 \\
9.00\end{array}$ & 6.4100 & $\begin{array}{l}4.6060 \\
4.60\end{array}$ & $\begin{array}{l}3.0560 \\
3.4000\end{array}$ & $\begin{array}{l}41.91 / 3339 \\
22.131800\end{array}$ & 79.361530 & $\begin{array}{r}0.300 \\
15.50\end{array}$ \\
\hline $\begin{array}{l}9.00 \\
5.00\end{array}$ & $\begin{array}{l}8.1100 \\
8.1200\end{array}$ & 4.6110 & 3.4000 & 72.280488 & 79.599974 & 22.50 \\
\hline 15.00 & 8.1200 & 4.6100 & 3.4000 & 72.257137 & 79.662676 & 32.50 \\
\hline 20.00 & 8.0100 & 4.5600 & 3.3700 & 70.074429 & 76.070675 & 50.00 \\
\hline $\begin{array}{l}20.00 \\
20.00\end{array}$ & 7.9500 & 4.5600 & 3.3700 & 70.074429 & 72.843563 & 70.00 \\
\hline $\begin{array}{l}20.00 \\
20.00\end{array}$ & 7.7100 & 4.4000 & 3.3700 & 65.243197 & 69.840218 & 90.00 \\
\hline $\begin{array}{l}20.00 \\
20.00\end{array}$ & 7.6800 & 4.3400 & 3.3300 & 62.722546 & 70.966294 & 110.00 \\
\hline 20.00 & 7.7770 & 4.3400 & 3.3300 & 62.722546 & 75.959060 & 130.00 \\
\hline 20.00 & 7.8500 & 4.3400 & 3.3300 & $62+722546$ & 79.757821 & 150.00 \\
\hline $\begin{array}{l}20.00 \\
20.00\end{array}$ & B. 1000 & 4.4500 & 3.3300 & 65.942324 & 86.596643 & 170.00 \\
\hline $\begin{array}{l}20.00 \\
20.00\end{array}$ & 8.1200 & 4.4500 & 3.3300 & 65.942324 & 87.676895 & 190.00 \\
\hline 20.00 & 8.1200 & 4.4500 & 3.3300 & 65.942324 & 87.676895 & 210.00 \\
\hline 20.00 & 8.1200 & 4.4500 & 3.3300 & 65.942324 & 87.576895 & 230.00 \\
\hline 20.00 & 8.1200 & 4.4500 & 3.3300 & 65.942324 & 87.676895 & 250.00 \\
\hline 20.00 & 8.1200 & 4.4500 & 3.3500 & 66.338373 & 88.203485 & 270.00 \\
\hline 20.00 & 8.1200 & 4.4500 & 3.3600 & 66.536399 & 88.466 .776 & 290.00 \\
\hline $\begin{array}{l}20.00 \\
20.00\end{array}$ & 8.1200 & 4.4500 & 3.3700 & 66.734424 & 88.730070 & 310.00 \\
\hline 20.00 & 8.1200 & 4.4500 & 3.3800 & 66.932448 & 88.993366 & 330.00 \\
\hline 20.00 & 8.2400 & 4.5000 & 3.3900 & 68.647499 & 92.877855 & 350.00 \\
\hline $\begin{array}{l}10.00 \\
20.00\end{array}$ & 3.3000 & 4.5300 & 3.4400 & 70.591894 & 95.797806 & 365.00 \\
\hline 20.00 & 3.3600 & 4.5600 & 3.5000 & 72.177597 & 99.058397 & 380.00 \\
\hline 25.00 & 8.7500 & 4. 7950 & 3.6840 & 84.702617 & 112.651014 & $402+50$ \\
\hline 20.00 & 9.1500 & 5.0400 & 3.8800 & 98.558205 & 127.726883 & 425.00 \\
\hline 10.00 & 7.4300 & 5.2170 & 3.9000 & 106.140643 & 134.513013 & 440.00 \\
\hline 20.00 & 9.7600 & 5.4000 & 3.9200 & 114.307198 & 144.795391 & 455.00 \\
\hline 25.00 & 9.7650 & 5.4000 & 3.9330 & 114.686278 & 145.659540 & 477.50 \\
\hline 25.00 & 9.7750 & 5.4000 & 3.9480 & 115.123677 & 146.986506 & 502.50 \\
\hline 25.00 & 9.7800 & 5.4000 & 3.9600 & 115.473598 & & $\begin{array}{l}527.50 \\
552.50\end{array}$ \\
\hline $\begin{array}{l}25.00 \\
25.00\end{array}$ & $\begin{array}{l}9.7840 \\
9.7480\end{array}$ & $\begin{array}{l}5.4000 \\
5.4000\end{array}$ & $\begin{array}{l}3.9880 \\
4.0220\end{array}$ & $\begin{array}{l}116.290077 \\
117,281517\end{array}$ & $\begin{array}{l}149.177738 \\
150.764442\end{array}$ & $\begin{array}{l}352.30 \\
577.50\end{array}$ \\
\hline 25.00 & 9.7920 & 5.4000 & 4.0560 & 118,272958 & 152.356596 & 602.50 \\
\hline 25.00 & 9.7960 & 5.4000 & 4.0900 & 119.264398 & 153.954199 & 627.50 \\
\hline 25.00 & 9.8000 & 5.4000 & 4.1200 & 120.139196 & 155.406395 & 652.50 \\
\hline 25.00 & 10.1630 & 5.6000 & 4.1650 & 130.614395 & 168.959763 & 677.50 \\
\hline 25.00 & & 5.8000 & 4.2120 & 141.691675 & 179.928814 & 702.50 \\
\hline 25.00 & 10.8180 & 6.1000 & 4.2570 & 158.402960 & 181.387039 & 727.50 \\
\hline 25.00 & 11.1200 & 6.2000 & 4.3000 & 165.291996 & 201.129906 & 752.50 \\
\hline 25.00 & 11.1350 & 6.2050 & 4.4750 & 172.296558 & 210.254181 & 777.50 \\
\hline 25.00 & 11.1500 & 6.2100 & 4.0 .330 & 178.667469 & 218.651192 & 802.50 \\
\hline 25.00 & 11.1650 & 6.2180 & 4.7970 & 185.468922 & 227.042854 & B2r.50 \\
\hline 25.00 & 11.1900 & 6.2300 & 4.9400 & 191.735720 & 233.991001 & 852.50 \\
\hline 25.00 & 11.2240 & 6.2500 & 4.9425 & 193.066404 & 236.514317 & 877.50 \\
\hline 25.00 & 11.2670 & 0.2750 & 4.9450 & 194.712463 & 238.319519 & 302.50 \\
\hline 25.00 & 11.3100 & 0.2970 & 4.9475 & 196.179302 & 240.506287 & 927.50 \\
\hline 25.00 & 11.3500 & 6.3220 & 4.9500 & 197.840031 & 241.991287 & 952.50 \\
\hline 25.00 & 11.3920 & 6.3400 & 4.9517 & 199.036549 & 244.546944 & 977.50 \\
\hline 25.00 & 11.4340 & 6.3600 & 4.9534 & 200,363043 & 240.063354 & 1002.50 \\
\hline 25.00 & 11.4760 & 6.3750 & 4.9550 & 201.374296 & 249.817837 & 1027.50 \\
\hline 25.00 & 11.5180 & 6.3900 & 4.9567 & 202.392468 & 252.792294 & 1052.50 \\
\hline 25,00 & 11.5600 & 6.4050 & 4.9584 & 203.413521 & 255.781784 & 1077.50 \\
\hline 25.00 & 11.6000 & 6.4210 & 4.9600 & 204.497020 & 258.423523 & 1102.50 \\
\hline
\end{tabular}

\begin{tabular}{|c|c|c|c|c|c|c|c|}
\hline 0 & ALPHA & BETA & RHO & MU & LAMOA & DEPIH & $M$ \\
\hline 10.00 & $\begin{array}{l}\text { AL } \\
0.1000\end{array}$ & 3.5400 & 2.5500 & 31.955579 & 30.974339 & 5.00 & 1 \\
\hline 6.50 & 6.1000 & 3.5400 & 2.5500 & 31.955579 & 30.974339 & 13.25 & 2 \\
\hline 5.00 & 6.4000 & 3.7000 & 3.0800 & 42.165199 & 41.826400 & 19.00 & 3 \\
\hline 5.00 & 6.7000 & 3.9200 & 3.4200 & 52.553087 & 48.417622 & 24.00 & 4 \\
\hline 5.00 & 6.7000 & 3.9200 & 3.4200 & 52.553087 & 48.417622 & 29.00 & 5 \\
\hline $\begin{array}{l}3.50 \\
5.00\end{array}$ & 6.7000 . & 3.9200 & 3.4200 & 52.553087 & 44.417622 & 33.25 & 6 \\
\hline $\begin{array}{r}5.00 \\
20.00\end{array}$ & $8.1500^{\circ}$ & 4.7500 & 3.4200 & 77.163750 & 72.837444 & 37.50 & 7 \\
\hline $\begin{array}{l}20.00 \\
20.00\end{array}$ & 8.1600 & 4.7500 & 3.4200 & 77.163750 & 73.395247 & 50.00 & a \\
\hline $\begin{array}{l}20.00 \\
20.00\end{array}$ & 8.2100 & 4.7500 & 3.4200 & 77.163750 & 76.194515 & 70.00 & 9 \\
\hline $\begin{array}{l}20.00 \\
20.00\end{array}$ & 8.2600. & 4.7500 & 3.4200 & 77.163750 & $79.0108 B 7$ & 90.00 & 10 \\
\hline $\begin{array}{l}20.00 \\
20.00\end{array}$ & B. 3200 & 4.7500 & 3.4200 & 77.163750 & 82.413103 & 110.00 & II \\
\hline 20.00 & B. 3000 & 4.7000 & 3.4000 & 75.105998 & 84.013996 & 130.00 & 12 \\
\hline 20.00 & 8.2840 & 4.5800 & 3.4000 & 71.319757 & 90.684305 & 150.00 & 13 \\
\hline 20.00 & 8.2840 & 4,5400 & 3.4000 & 70.079437 & 93.164946 & 170.00 & 14 \\
\hline 20.00 & 8.2840 & 4.5400 & 3.4100 & 70.285554 & 93.438961 & 190.00 & 15 \\
\hline $\begin{array}{l}20.00 \\
20.00\end{array}$ & 8.2840 & 4.5400 & 3.4200 & 70.491670 & 93.712975 & 210.00 & 16 \\
\hline 20.00 & 8.2840 & 4.5400 & 3.4500 & 71.110018 & 94.535019 & 230.00 & 17 \\
\hline 20.00 & 8.2840 & 4.5400 & 3.4500 & 71.110018 & 94.535019 & 250.00 & 18 \\
\hline 20.00 & $8.2840^{\circ}$ & 4.5400 & 3,4500 & 71.110018 & 94.535019 & 270.00 & 19 \\
\hline 20.00 & 8.2840 & 4.5400 & 3.4500 & 71.110018 & 94.535019 & 290.00 & 20 \\
\hline 20.00 & 8.2840 & 4.5400 & 3.4500 & 71.110018 & 94.535019 & 310.00 & 21 \\
\hline 20.00 & 8.2840 & $4 \star 5400$ & 3.4500 & 71.110018 & 94.535019 & 330.00 & 22 \\
\hline 20.00 & 8.3130 & 4.5400 & 3.4500 & 71.110018 & 96.195551 & 350.00 & 23 \\
\hline 10.00 & 8.5070 & 4.6430 & 3.4500 & 74.373197 & 100.926821 & 365.00 & 24 \\
\hline $\begin{array}{l}20.00 \\
25.00\end{array}$ & 8.7000 & 4.7500 & 3.4500 & 77.840624 & 105.449245 & 380.00 & 25 \\
\hline $\begin{array}{l}25.00 \\
20.00\end{array}$ & 8.7400 & 4.7500 & 3.6600 & 82.578750 & 114.421106 & 402.50 & 26 \\
\hline $\begin{array}{l}20.00 \\
10.00\end{array}$ & 8.7600 & 4.7500 & 3.8800 & 87.542500 & 122.656883 & 425.00 & 27 \\
\hline $\begin{array}{l}10.00 \\
20.00\end{array}$ & 9.0380 & 5.0000 & 3.9000 & 97.499999 & 123.573225 & 440.00 & $2 \theta$ \\
\hline $\begin{array}{l}20.00 \\
25.00\end{array}$ & 9.4890 & 5.2500 & 3.9200 & 108.044999 & 136.871167 & 455.00 & 29 \\
\hline $\begin{array}{l}25.00 \\
25.00\end{array}$ & 9.5000 & 5.2530 & 3.9330 & 108.527235 & 137.898777 & 477.50 & 30 \\
\hline $\begin{array}{l}25.00 \\
25.00\end{array}$ & 9.5160 & 5.2570 & 3.9480 & 109.107119 & 139.293953 & 502.50 & 31 \\
\hline $\begin{array}{l}25.00 \\
25.00\end{array}$ & 9.5260 & 5.2600 & 3.9600 & 109.563694 & 140.221516 & 527.50 & 32 \\
\hline $\begin{array}{l}25.00 \\
25.00\end{array}$ & 9.5760 & 5.2850 & 3.9880 & 111.389723 & 142.919250 & 552.50 & 33 \\
\hline $\begin{array}{l}25.00 \\
25.00\end{array}$ & 9.6300 & 5.3130 & 4.0220 & 113.532887 & 145.922029 & 577.50 & 34 \\
\hline $\begin{array}{l}25.00 \\
25.00\end{array}$ & 9.6830 & 5.3400 & 4.0560 & 115.659271 & 148.973991 & 602.50 & 35 \\
\hline $\begin{array}{l}25.00 \\
25.00\end{array}$ & 9.7360 & 5.3670 & 4.0900 & 117.811175 & 152.067495 & 627.50 & 36 \\
\hline $\begin{array}{l}25.00 \\
25.00\end{array}$ & 9.7820 & $5: 3900^{\circ}$ & 4.1200 & 119.694649 & 154.843288 & 652.50 & 37 \\
\hline $\begin{array}{l}25.00 \\
25.00\end{array}$ & 10.0140 & 5.5180 & 4.1650 & 126.817265 & 164.032475 & 677.50 & 38 \\
\hline $\begin{array}{l}25.00 \\
25.00\end{array}$ & 10.1800 & 5.6300 & 4.2120 & 133.507339 & $169.48497 \theta$ & 702.50 & 39 \\
\hline $\begin{array}{l}25.00 \\
25.00\end{array}$ & 10.1900 & 5.7460 & 4.2570 & 140.551306 & 160.927654 & 727.50 & 40 \\
\hline $\begin{array}{l}25.00 \\
25.00\end{array}$ & 10.4920 & 5.8500 & 4.3000 & 147.156744 & 179.039375 & $\begin{array}{l}752.50 \\
717.50\end{array}$ & 41 \\
\hline $\begin{array}{l}25.00 \\
25.00\end{array}$ & 10.6770 & 5.9500. & 4.4750 & 258.426184 & 193.290142 & $\begin{array}{l}777.50 \\
802.50\end{array}$ & 42 \\
\hline $\begin{array}{l}25.00 \\
25.00\end{array}$ & 16.8520 & $6.0440^{\circ}$ & $4.6330^{\circ}$ & 169.243189 & $\begin{array}{l}207.123043 \\
221.388382\end{array}$ & $\begin{array}{l}802.50 \\
827.50\end{array}$ & $\begin{array}{l}43 \\
44\end{array}$ \\
\hline $\begin{array}{l}25.00 \\
25.00\end{array}$ & 11.0250 & 6.1400 & $\begin{array}{l}4.7970 \\
4.9400\end{array}$ & $\begin{array}{r}180.844978 \\
191.735720\end{array}$ & $\begin{array}{l}221.588381 \\
233.991001\end{array}$ & $\begin{array}{l}827.50 \\
852.50\end{array}$ & $\begin{array}{l}44 \\
45\end{array}$ \\
\hline $\begin{array}{l}25.00 \\
25.00\end{array}$ & 11.1800 & 6.2300 & $\begin{array}{l}4.9400 \\
4.9425\end{array}$ & $\begin{array}{l}191.735720 \\
193.066404\end{array}$ & 236.514317 & 877.50 & 46 \\
\hline $\begin{array}{l}25.00 \\
25.00\end{array}$ & 11.2240 & $\begin{array}{l}6.2500 \\
6.2750\end{array}$ & $\begin{array}{l}4.9425 \\
4.9450\end{array}$ & 194.712463. & 238.319519 & 902.50 & 47 \\
\hline $\begin{array}{l}25.00 \\
25.00\end{array}$ & 11.2670 & $\begin{array}{l}6.2750 \\
6.2970\end{array}$ & 4.9475 & 196.179302 & 240.506287 & 927.50 & 48 \\
\hline $\begin{array}{l}25.00 \\
25.00\end{array}$ & $\ldots . .+11.3100$ & $\begin{array}{l}6.2910 \\
0.3220\end{array}$ & 4.9500 & 197.340031 & 241.991287 & 952.50 & 49 \\
\hline 25.00 & 11.3920 & 6.3400 & 4.9517 & 199.036549 & 244.546944 & 977.50 & 50 \\
\hline 25.00 & 11.4340 & 6.3600 & 4.9534 & 200.363043 & 246.863354 & 1002.50 & 51 \\
\hline 25.00 & 11.4760 & 8.3750 & 4.9550 & 201.374296 & 249.817037 & 1.027 .50 & 52 \\
\hline 25.00 & 11.5180 & 5.3900 & 4.9567 & 202.392466 & 252.792294 & 1052.50 & 53 \\
\hline 25.00 & 11.5600 & 0.4050 & 4.9584 & 203.413521 & 255.781784 & 1077.50 & 54 \\
\hline 25.00 & II. 6000 & 6.4210 & 4.9600 & 204.497028 & 258.423523 & 1102.50 & 5.5 \\
\hline
\end{tabular}


TABLE 3

Phase Velocity, Group Velocity, and Spectral-amplitude Response of the First Three: Rayleigh-Wave Modes and the First Four Love-wave Modes for an Oceanic Modei

OCEAN

RAYLEIGH

LOVE

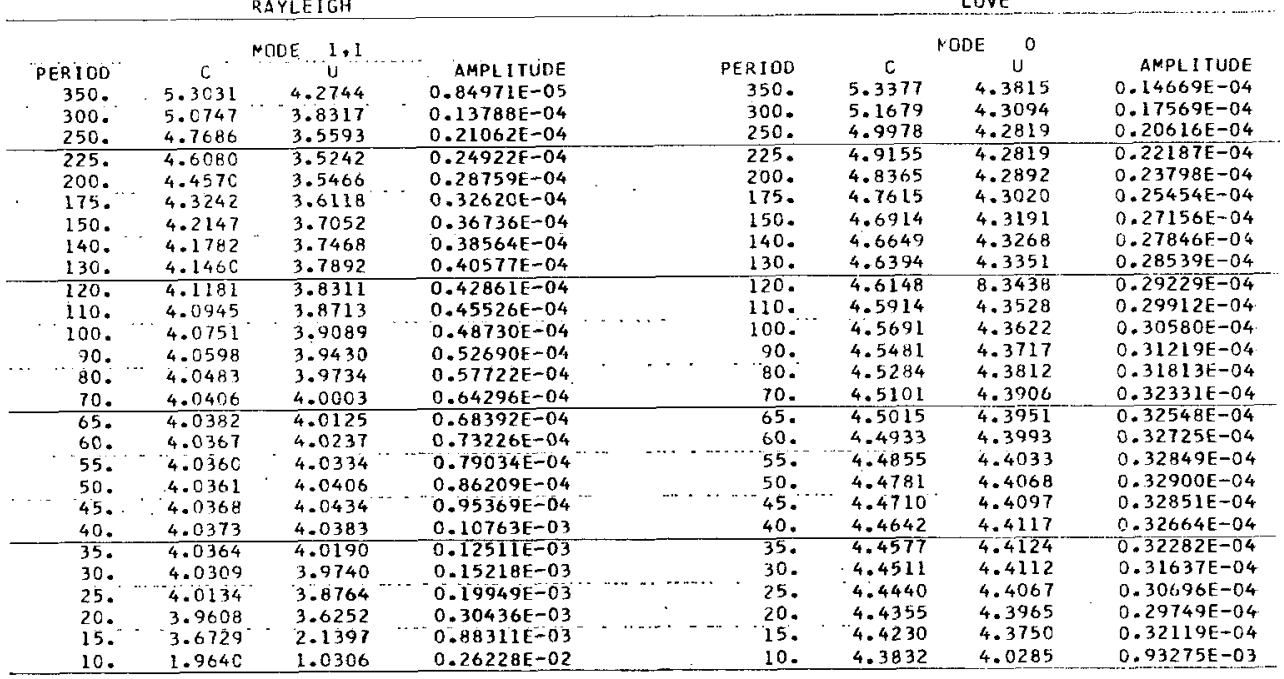

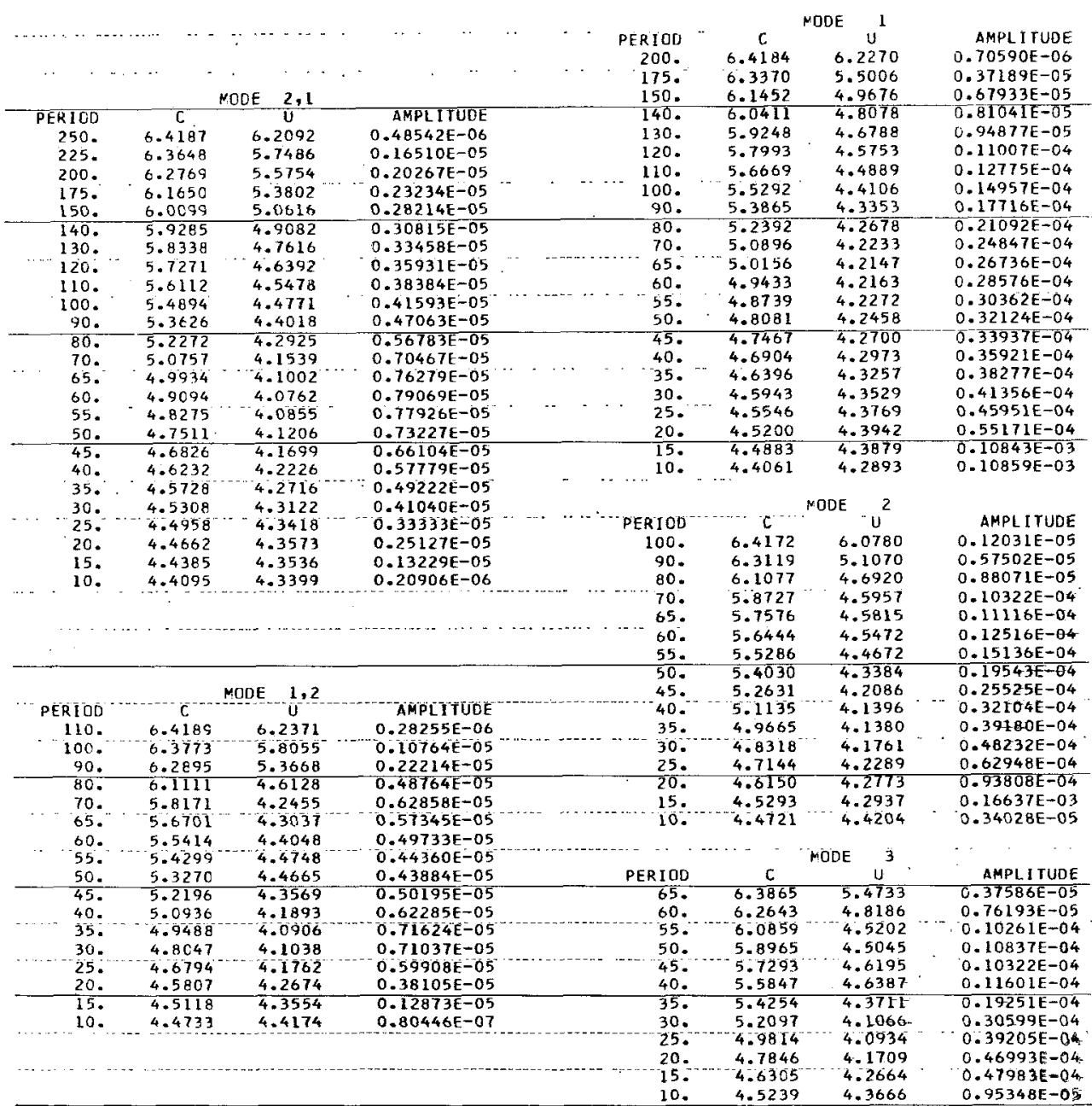


TABLE 4

Phase Velocity, Group Velocity, and Spectral-amplitude Response of the First Three Rayleigh-Wave Modes and the First Four love-wave Modes for a Shield Model

SHIELO

RAYLEIGH

LOVE

\begin{tabular}{|c|c|c|c|c|c|c|c|}
\hline \multicolumn{4}{|c|}{ MODE 1,1} & \multicolumn{4}{|c|}{ MODE $\quad 0$} \\
\hline PERIOD & $c^{-}$ & u & AMPL I TUDE & PERIOD & c & $u$ & AMPLITUDEE \\
\hline 350. & 5.2358 & 4.1434 & $0.90302 \mathrm{E}-05$ & 350. & 5.3418 & 4.4623 & $0.13407 E-04$ \\
\hline 300. & 5.0010 & 3.7837 & $0.13786 E-04$ & 300. & 5.1893 & 4.4080 & $0.15889 E-04$ \\
\hline 250. & 4.7184 & 3.6251 & $0.19418 \mathrm{E}-04$ & 250. & 5.0377 & 4.3896 & $0.18636 E-04$ \\
\hline 225. & $\frac{7.1707}{4.5800}$ & 3.6302 & $0.22226 \mathrm{E}-04$ & 225 & 4.9644 & 4.3911 & $0.20169 \mathrm{k}=04$ \\
\hline 200. & 4.4542 & 3.6771 & $0.25009 E-04$ & 200. & 4.8939 & 4.3976 & $0.21881 E-04$ \\
\hline 175. & 4.3461 & 3.7541 & $0.27862 E-04$ & 175. & 4.8265 & 4.4074 & $0.23881 E-04$ \\
\hline 150. & 4.2573 & 3.8512 & $0.30976 E-04$ & 150. & 4.7628 & 4.4186 & $0.26369 E-04$ \\
\hline 140. & 4.2312 & 3.8934 & $0.32378 E-04$ & 140. & 4.7384 & 4.4230 & $0.27585 \mathrm{E}-04$ \\
\hline 130. & 4.2069 & 3.9361 & $0.33936 E-04$ & 130. & 4.7145 & 4.4271 & $0.28990 \mathrm{E}-04$ \\
\hline 120 & 4.1867 & 3.9783 & $0.35717 E-04$ & 120. & 4.6913 & 4.4305 & $0.30657 E-04$ \\
\hline 110. & 4.1704 & 4.0187 & $0.37825 E-04$ & 110. & 4.6685 & 4.4330 & $0.32700 E-04$ \\
\hline 100. & 4.1580 & 4.0555 & $0.40416 E-04$ & 100. & 4.6461 & 4.4338 & $0.35314 E-04$ \\
\hline 90. & 4.1492 & 4.0870 & $0.43737 F-04$ & 90. & 4.6239 & 4.4317 & $0.38845 E-04$ \\
\hline 80. & 4.1437 & 4.1097 & $0.48207 \mathrm{E}-04$ & 80. & 4.6013 & 4.4244 & $0.43977 E-04$ \\
\hline 70. & 4.1402 & 4.1200 & $0.54557 \mathrm{E}-04$ & 70. & 4.5774 & 4.4066 & $0.52200 \mathrm{E}-04$ \\
\hline 65. & 4.1387 & 4.1182 & $0.58850 \mathrm{E}-04$ & 65. & 4.5641 & 4.3904 & $0.58459 \mathrm{E}-04$ \\
\hline & & 4.1102 & & & 4.5493 & 4.3658 & $0.67215 \mathrm{E}-04$ \\
\hline 55. & 4.1340 & $\begin{array}{l}4.01023 \\
4.0939\end{array}$ & $0.71266 \mathrm{E}-04$ & 55. & $\begin{array}{r}4.5493 \\
4.5318\end{array}$ & $\begin{array}{l}4.3279 \\
4.3279\end{array}$ & $0.799 B 2 E-04$ \\
\hline 50. & 4.1291 & 4.0664 & $0.80633 E-04$ & 50. & 4.5097 & 4.2692 & $0.99298 \mathrm{E}-04$ \\
\hline 45. & 4.1206 & 4.0234 & $0.93661 \mathrm{E}-04$ & 45. & 4.4796 & 4.1802 & $0.12904 E-03$ \\
\hline 40. & 4.1059 & 3.9585 & $0.11271 \mathrm{E}-03$ & 40. & 4.4363 & 4.0555 & $0.17378 E-03$ \\
\hline 35. & 4.0804 & 3.8614 & $0.14244 \mathrm{t}-03$ & 35. & 4.3732 & 3.9044 & $0.23707 \mathrm{E}=03$ \\
\hline 30. & 4.0362 & 3.7160 & $0.19288 E-03$ & 30. & 4.2849 & 3.7488 & $0.32192 \mathrm{E}-03$ \\
\hline 25. & 3.9578 & 3.5018 & $0.28737 \mathrm{E}-03$ & 25. & 4.1691 & $3,611 \mathrm{I}$ & $0.43288 E-03$ \\
\hline 20. & 3.8204 & 3.2278 & $0.47539 E-03$ & 20. & 4.0296 & 3.5126 & $0.57359 \mathrm{E}-03$ \\
\hline $150^{\circ}$ & 3.6112 & 3.0266 & $0.81247 E-03$ & 15. & 3.8777 & $3.469 \mathrm{I}$ & $0.74586 \mathrm{E}-03$ \\
\hline 10. & 3.3885 & 3.0400 & $0.13263 \mathrm{~F}-02$ & 10. & 3.7314 & 3.4754 & $0.96615 E-03$ \\
\hline
\end{tabular}

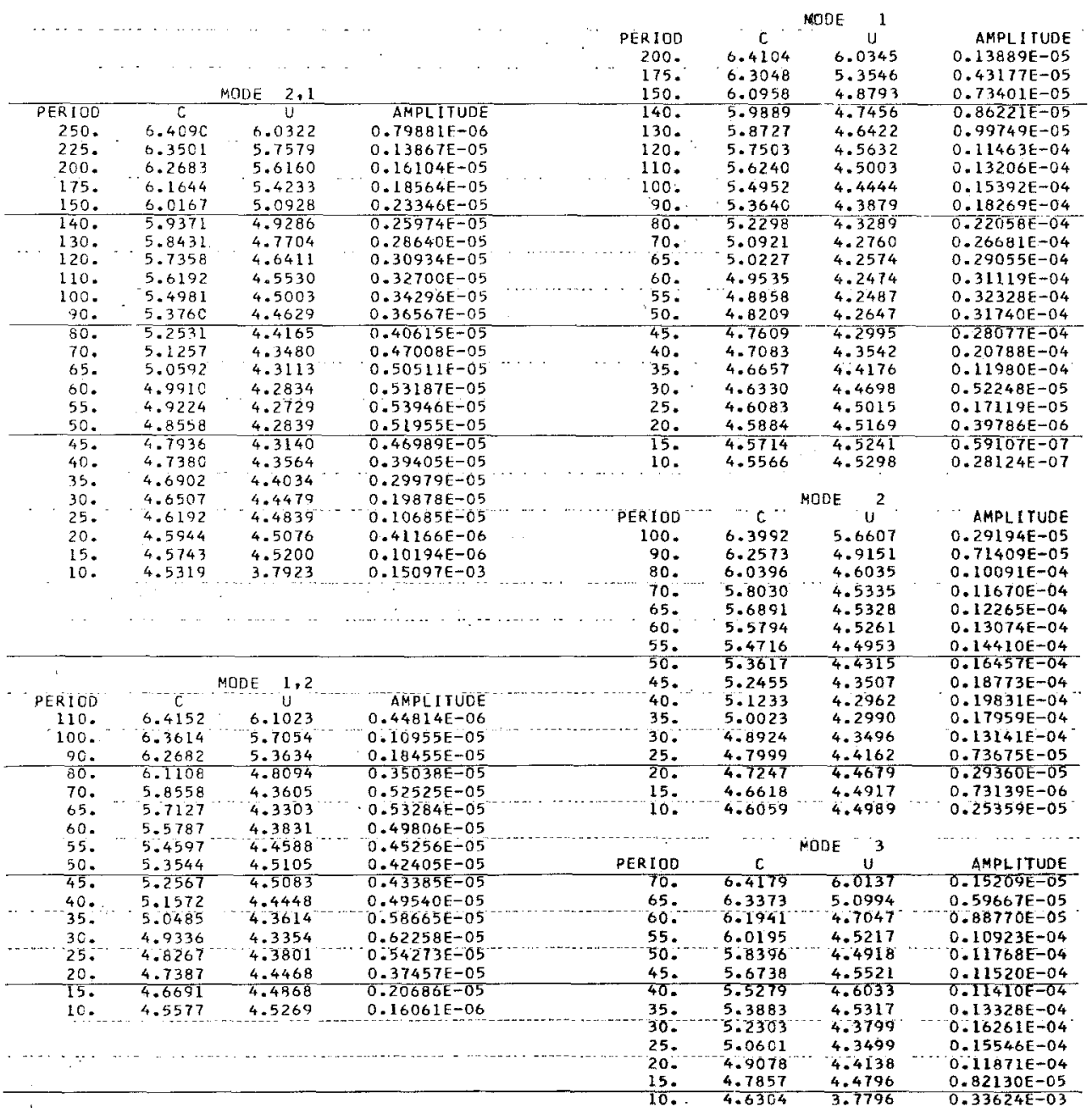


vector, spectral values below or above the tabulated depths can be obtained by continued multiplication of the vector by the Thomson-Haskell matrix or its inverse, respectively, to the desired depths. The accuracy of the results can be estimated by

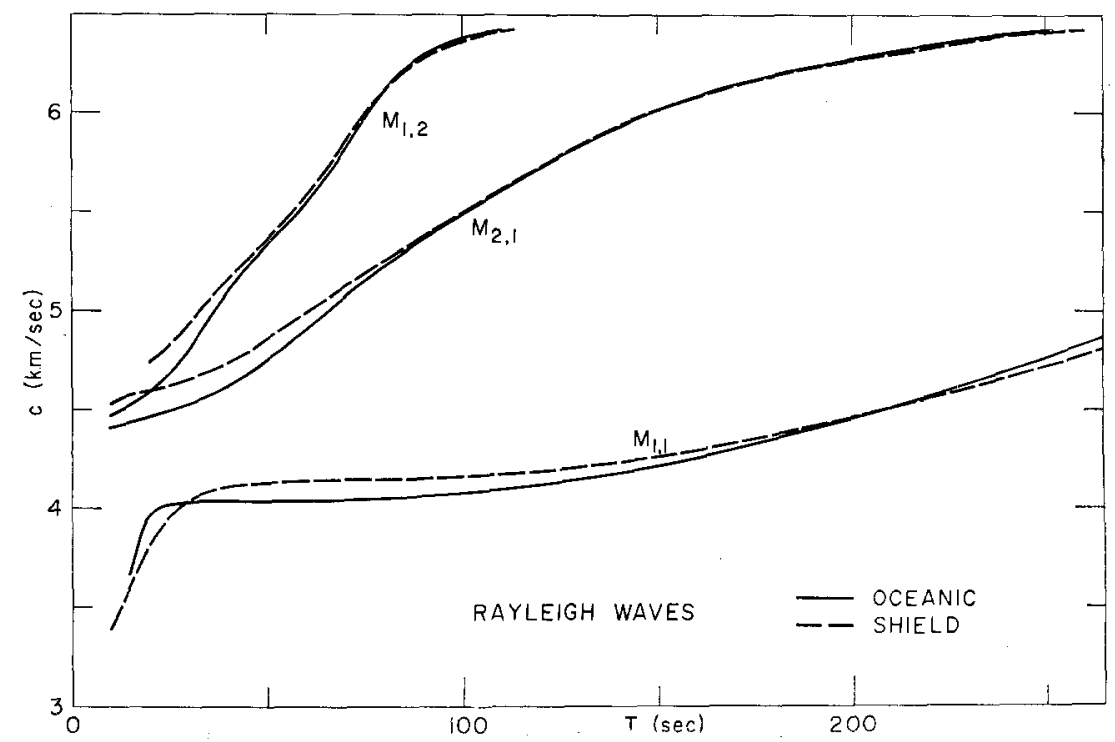

FIG. 2. Rayleigh-wave phase-velocity curves for the fundamental and two higher modes for the oceanic and shield models.

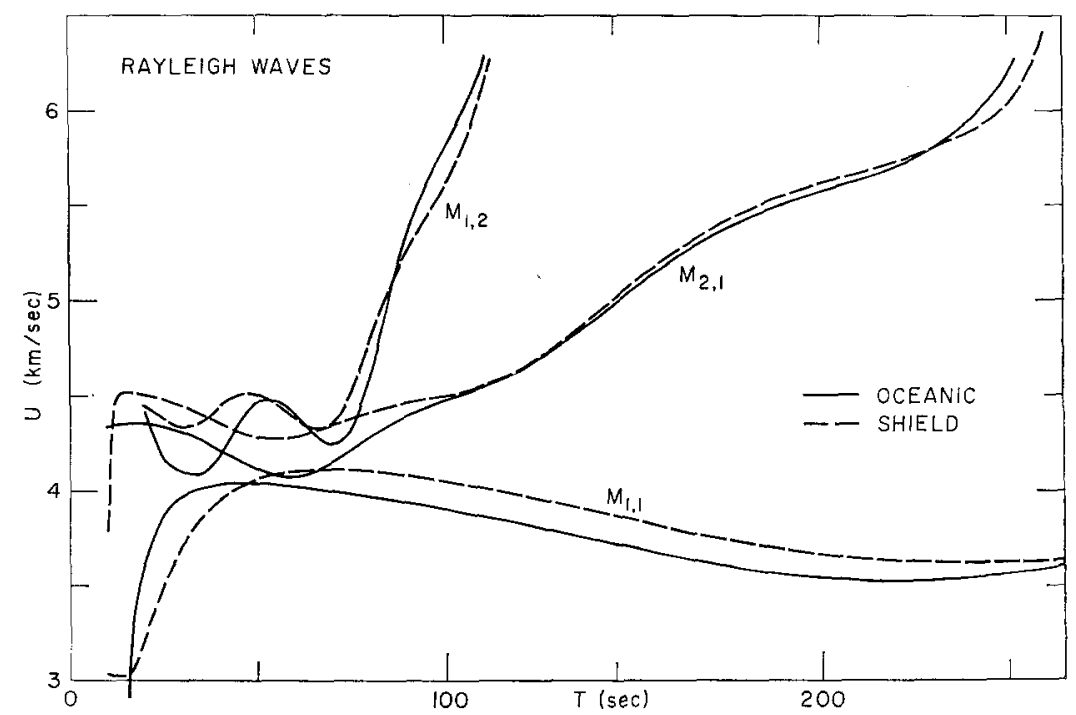

FIG. 3. Rayleigh-wave group-velocity curves for the fundamental and two higher modes for the oceanic and shield models.

comparing two sets of the same intermediate depth values where one set is obtained by downward continuation and the other by upward continuation.

\section{Spectral Ratios}

Using arguments based on the reciprocity theorems of Knopoff and Gangi (1959), Aki (personal communications, 1961) realized that the vertical surface displacement 
spectrum of fundamental Rayleigh waves should possess a zero which was dependent on the depth of a horizontal force. This phenomenon was investigated in a series of model experiments (Aki and Healy, personal communications, 1962). Harkrider and

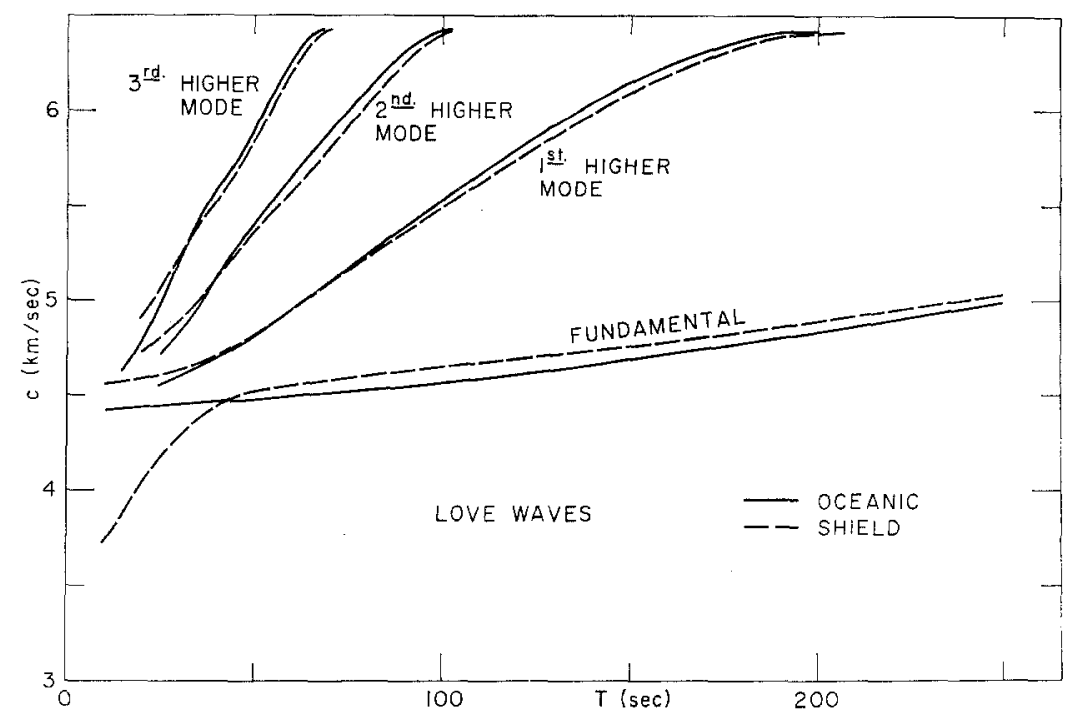

Frg. 4. Love-wave phase-velocity curves for the fundamental and three higher modes for the oceanic and shield models.

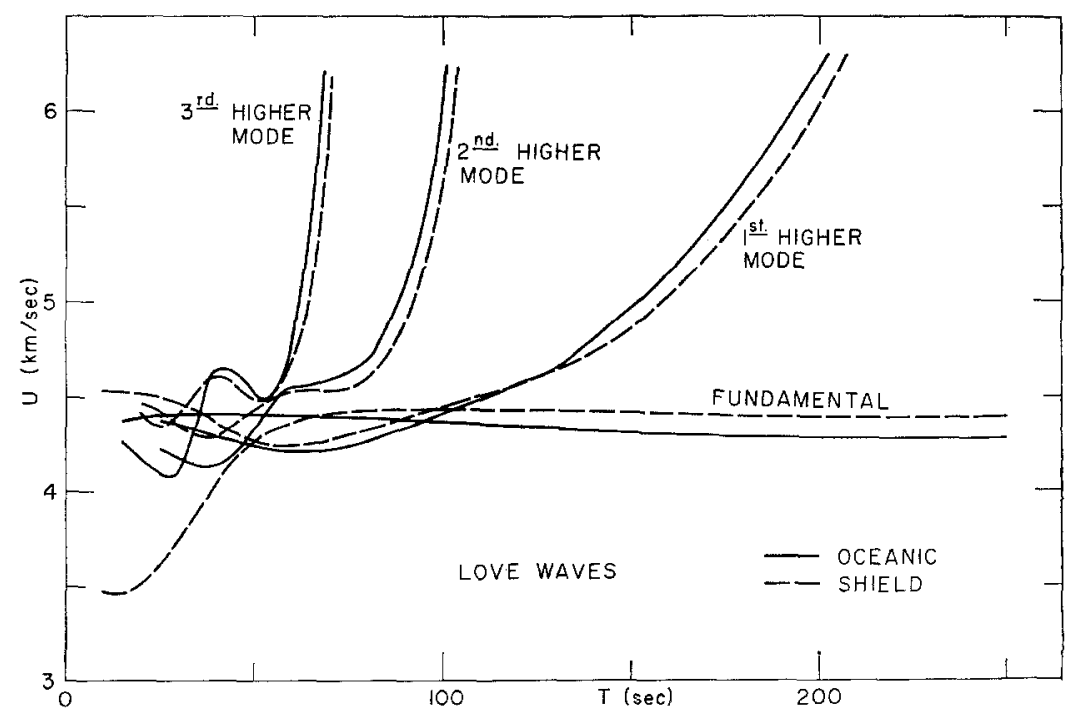

FIG. 5. Love-wave group-velocity curves for the fundamental and three higher modes for the oceanic and shield models.

Anderson (1966) noted that the higher-mode Rayleigh and Love waves possess depthdependent spectral zeros for vertical as well as horizontal forces.

One of the difficulties encountered in using amplitude spectra for depth determination has been the time-space source function. This function will, in general, have zeros associated with the fault displacement-time function, fault dimensions, and rupture velocity. For stationary point sources, the source factor can be eliminated by dividing 
the spectra of different arrivals. This division eliminates all of the effects of distance except the difference in anelastic absorption. For more realistic sources complications arise which will be discussed later.

For small earthquakes, the departure from point-source theory is negligible for periods greater than 20 sec. Tsai (1969) found that the assumption that the source is a

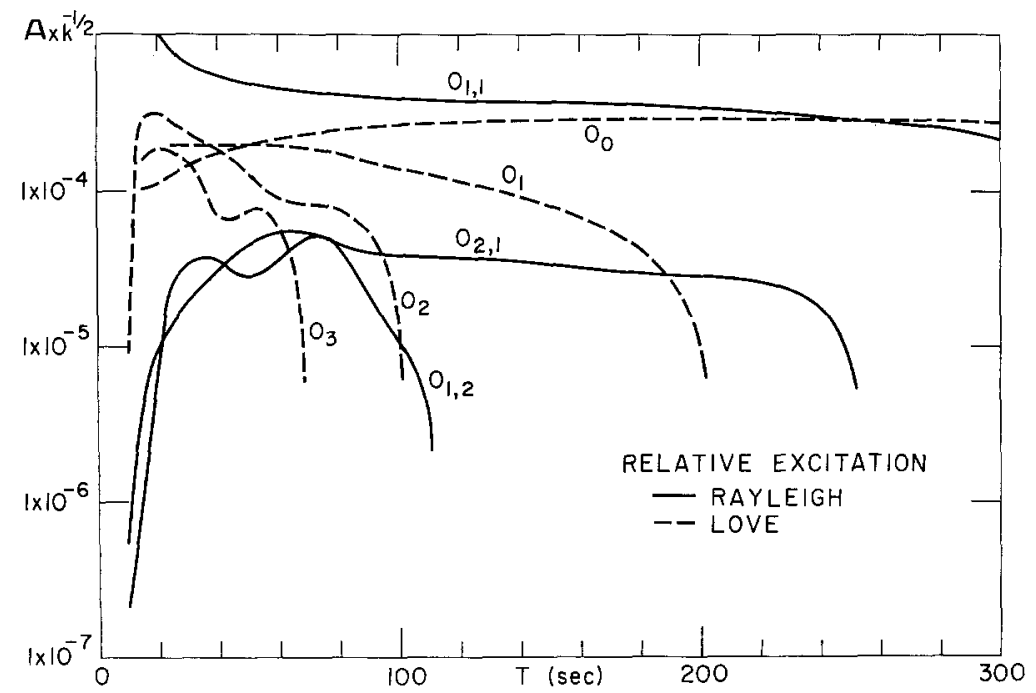

FIG. 6. Relative spectral excitation for the oceanic structure in units of $10^{-12.5} \mathrm{~cm}^{3 / 2} /$ dyne.

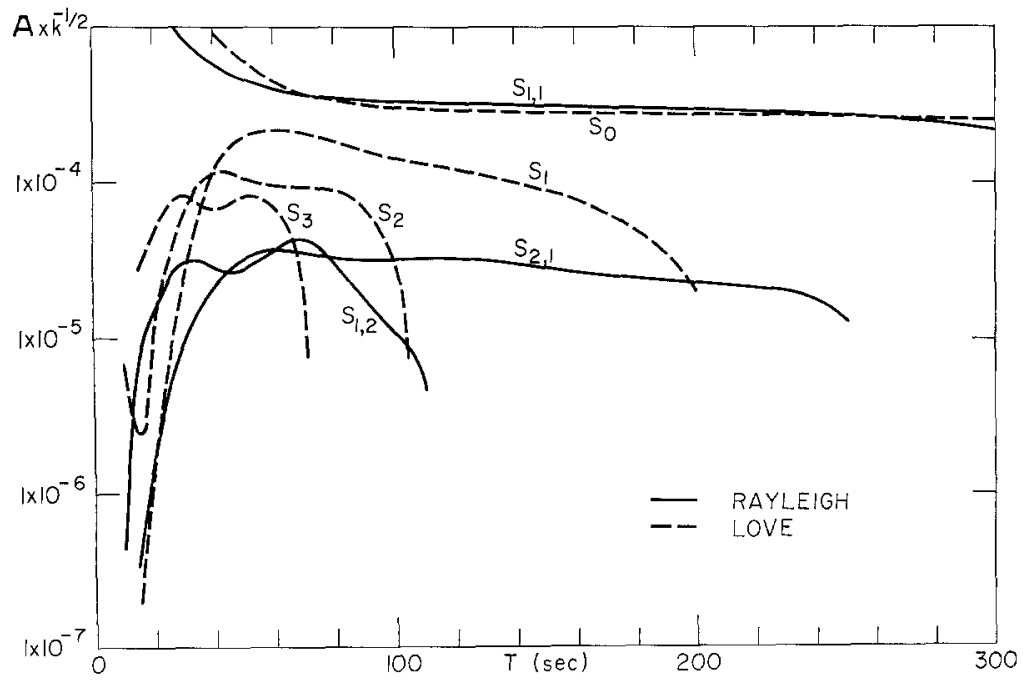

FIG. 7. Relative excitation for the shield structure in units of $10^{-12 \cdot 5} \mathrm{~cm}^{3 / 2} /$ dyne.

point in space and a step function in time was adequate for determining the focal depths of earthquakes in the mid-ocean ridges with magnitudes 6.0 or smaller. The depths were determined from the shapes of the Rayleigh-wave spectra by comparing with theoretical spectra from a vertical strike slip fault with a step function rupture in time. The depth estimates were confirmed by observation of $P$ and $p P$ phases.

In order to investigate the use of spectral ratios as a possible measure of source 
TABLE 5

Surface Ellipticity of the Firgt Three Rayleigh-Waye Modes for the Oceanic and Shield MODELS

OCEAN

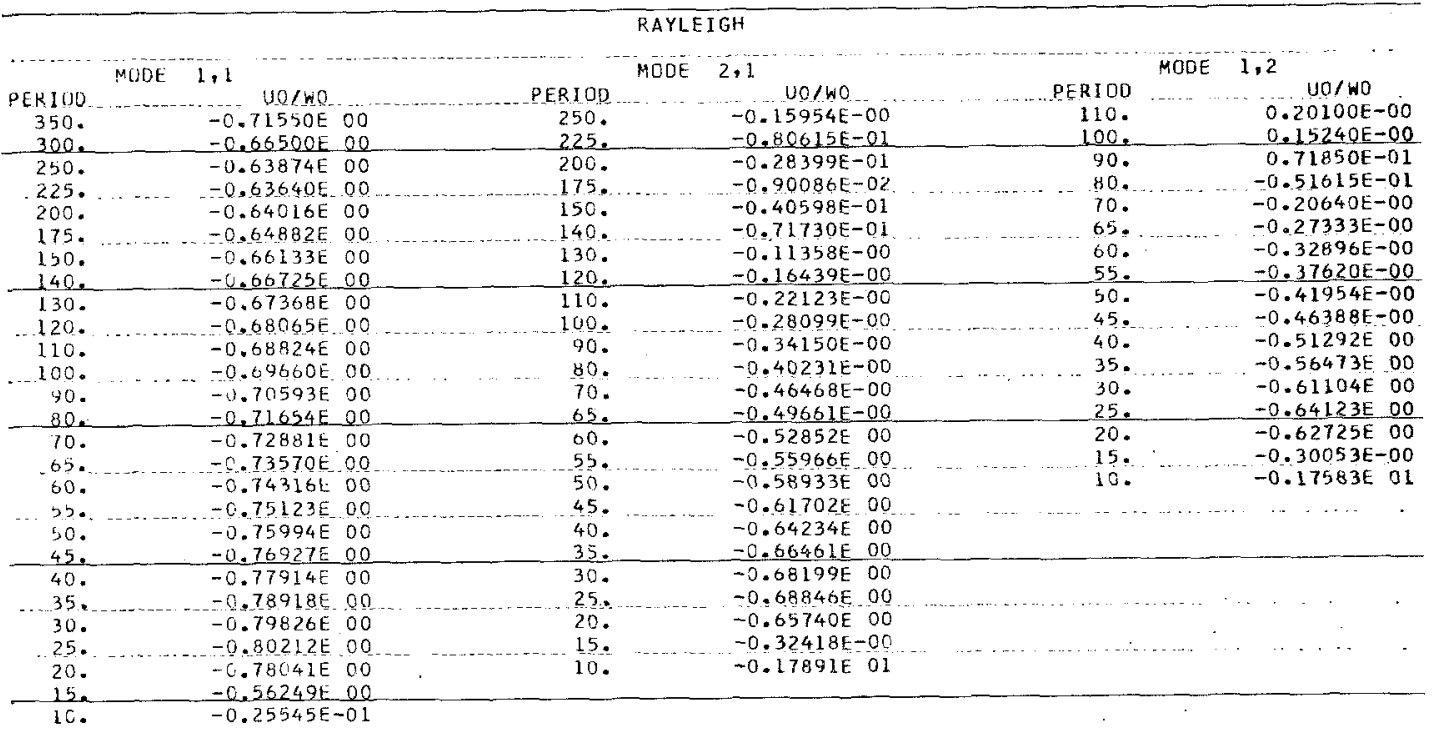

SHIELD

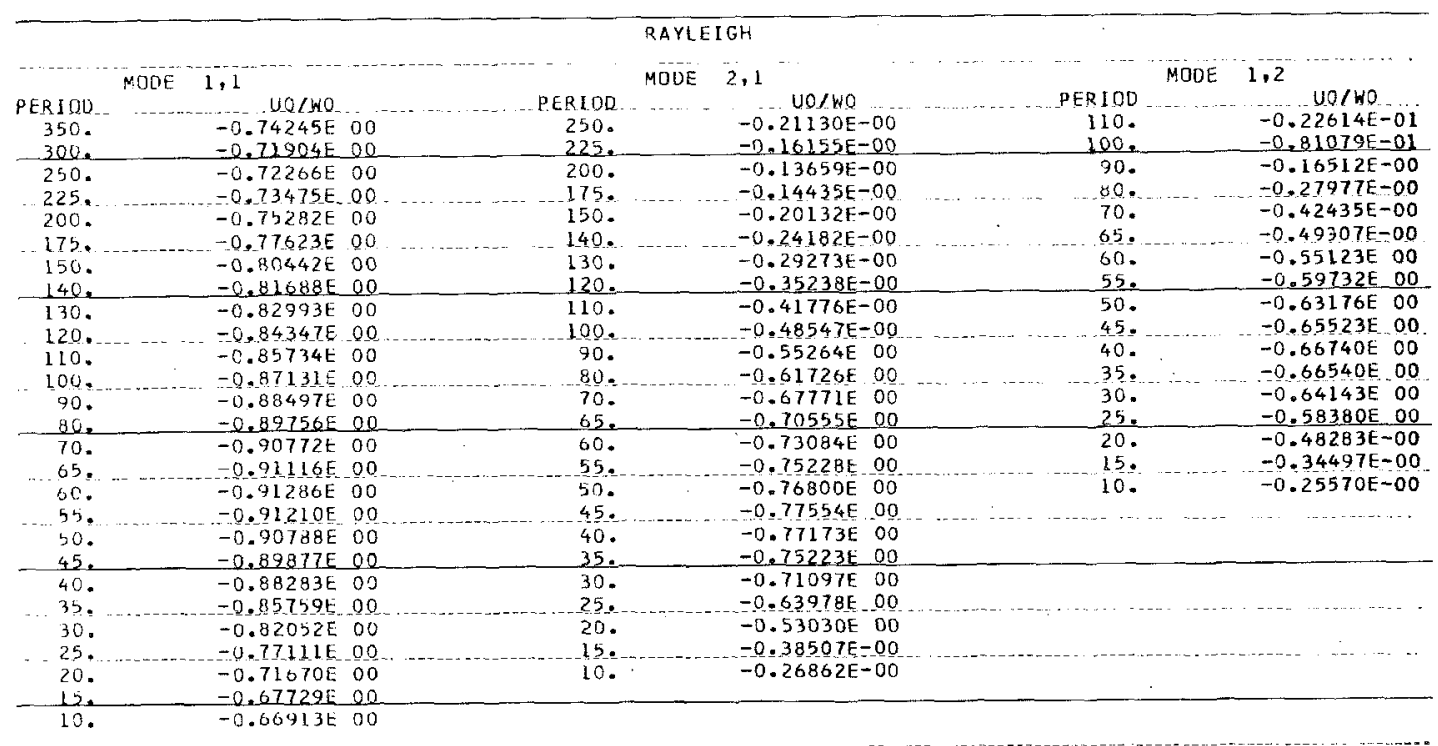

depth, we have evaluated the ratio of various surface-wave modes as a function of period and depth. The calculations are for a double-couple source with an oceanic propagation path. The orientation of the double couple was chosen in order to represent the far-field spectra from a vertical strike-slip fault. The station aximuth is $22.5^{\circ}$ from the fault plane.

The ratio of fundamental Rayleigh to Love spectra are shown in Figure 8. The ratio 
TABLE 6

Eigenfunctions for the Rayleigh- and Love-Wave Modes at a Depth of $10 \mathrm{~km}$ in the OCEANIC MODEL

DCEAN




TABLE 7

SHIELD.

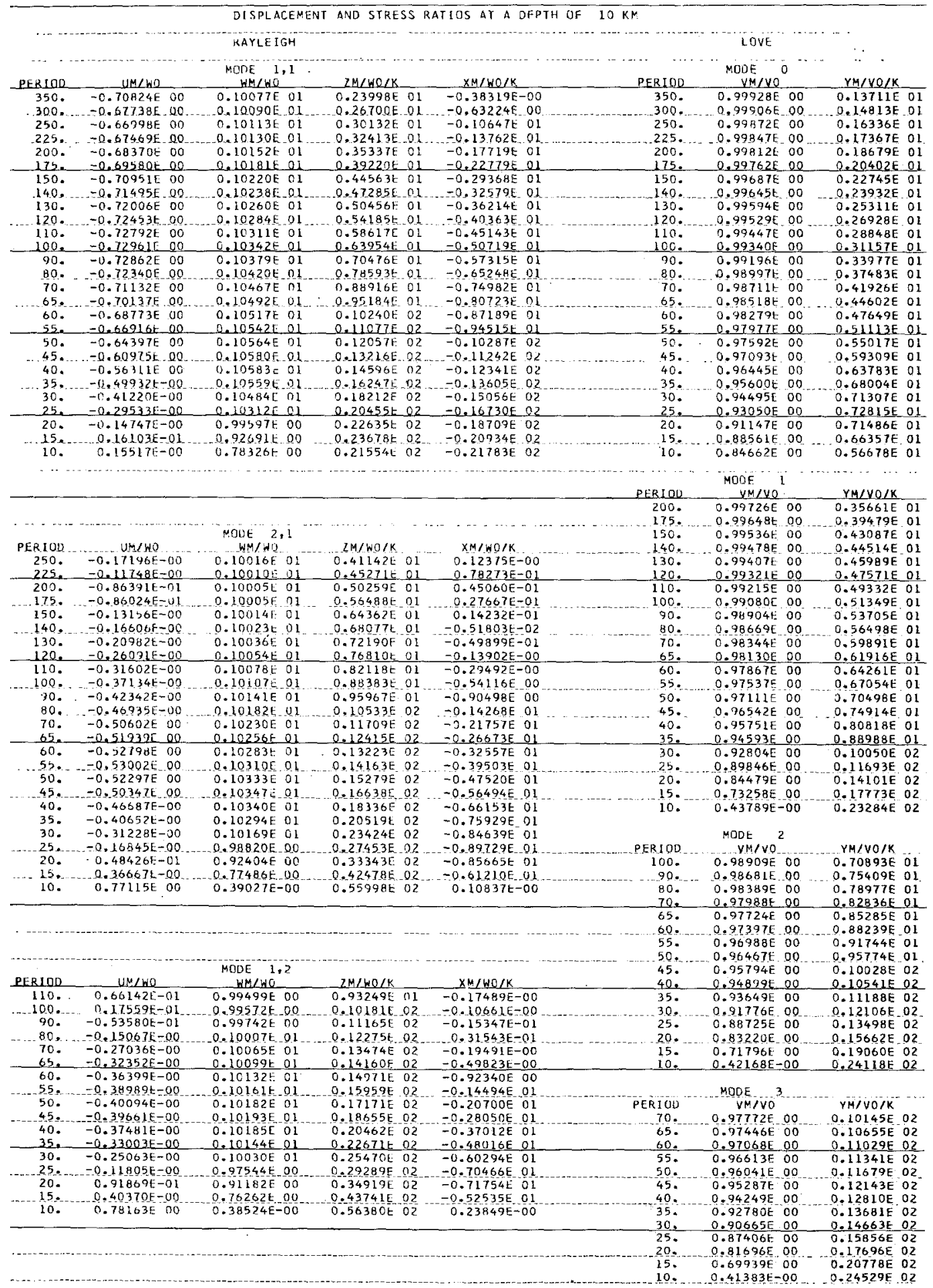


TABLE 8

DCEAN

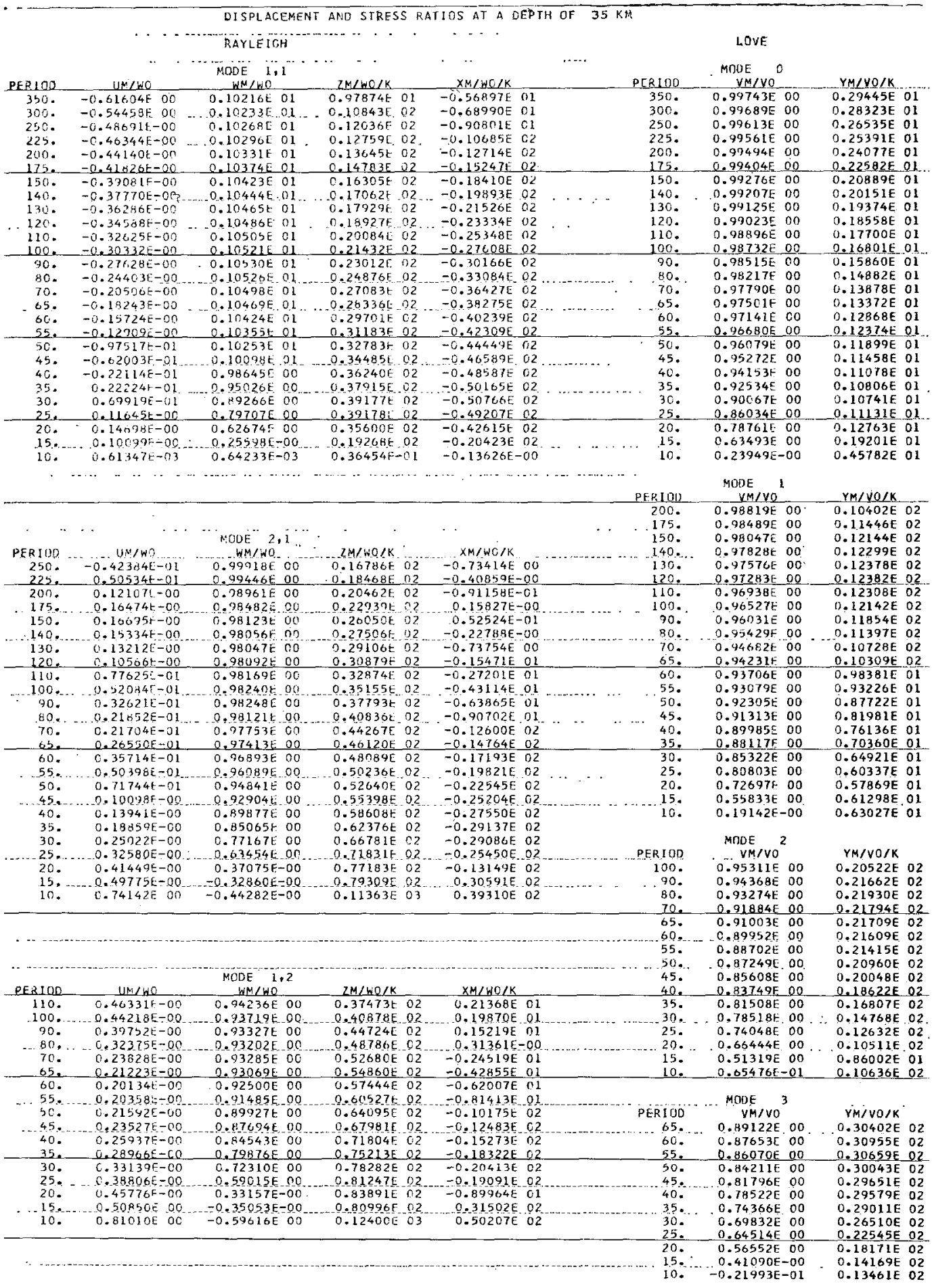


TABLE 9

SHIELD

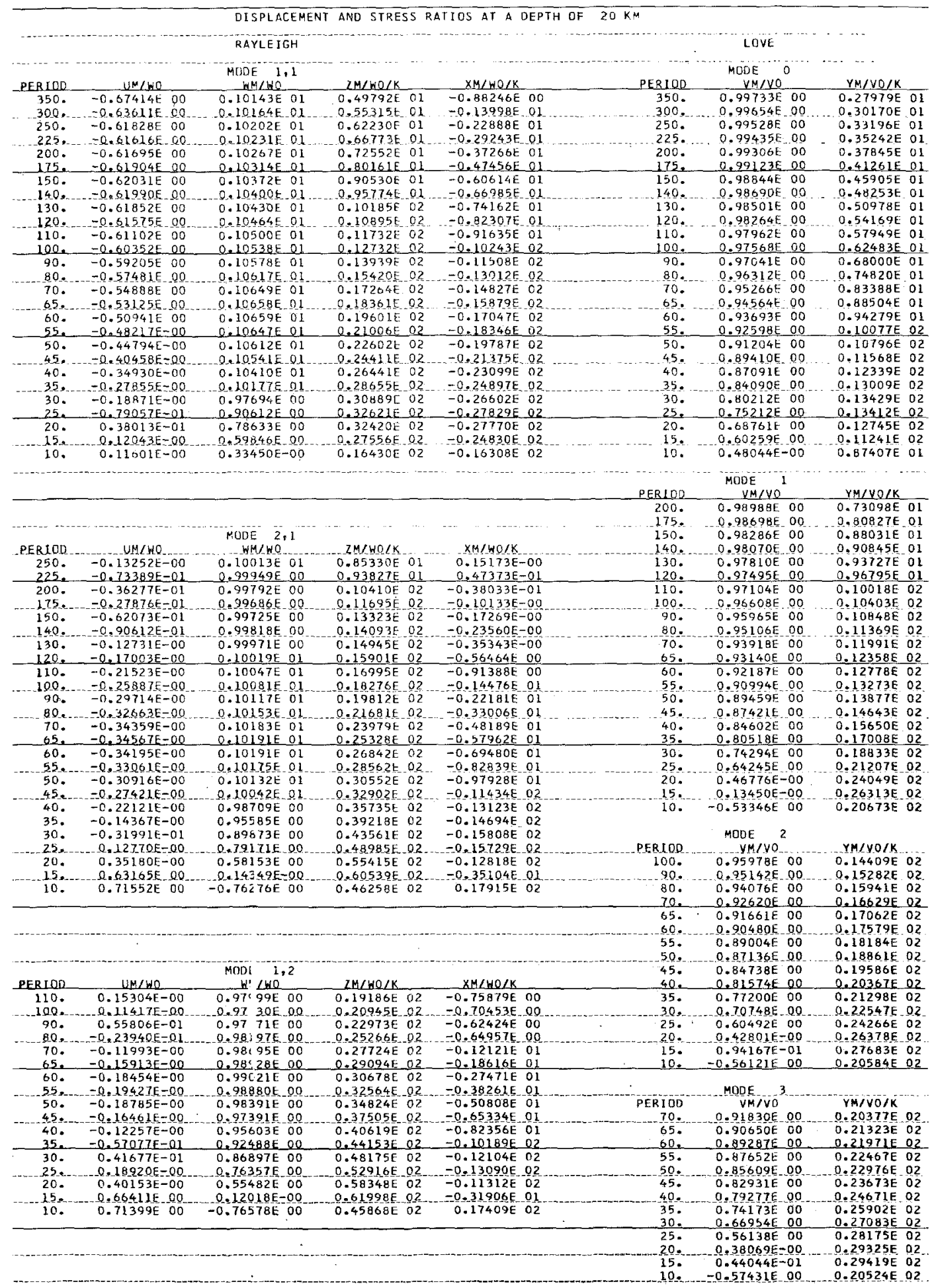


TABLE 10

OCEAYS

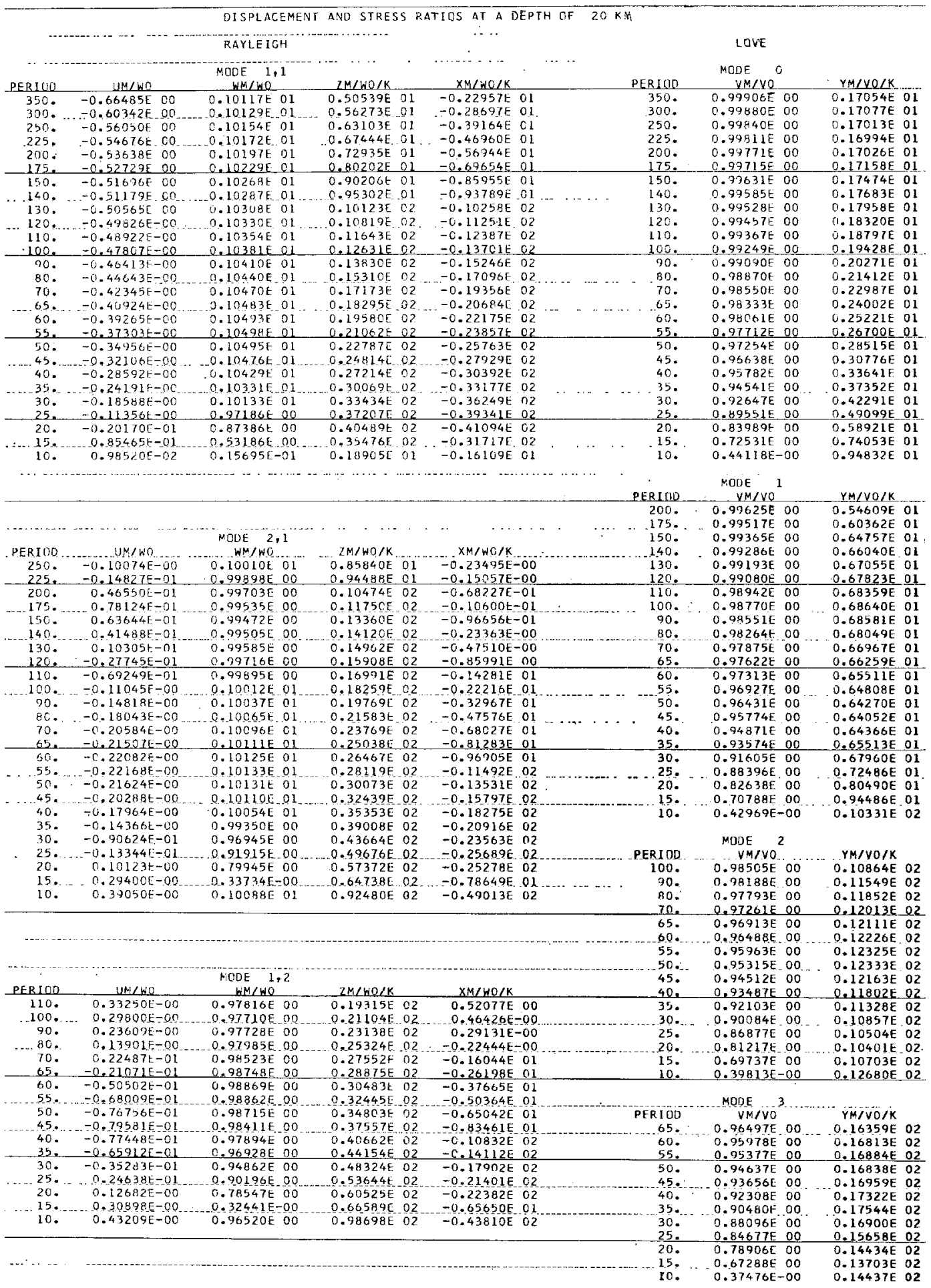


TABLE 11

SHIELD

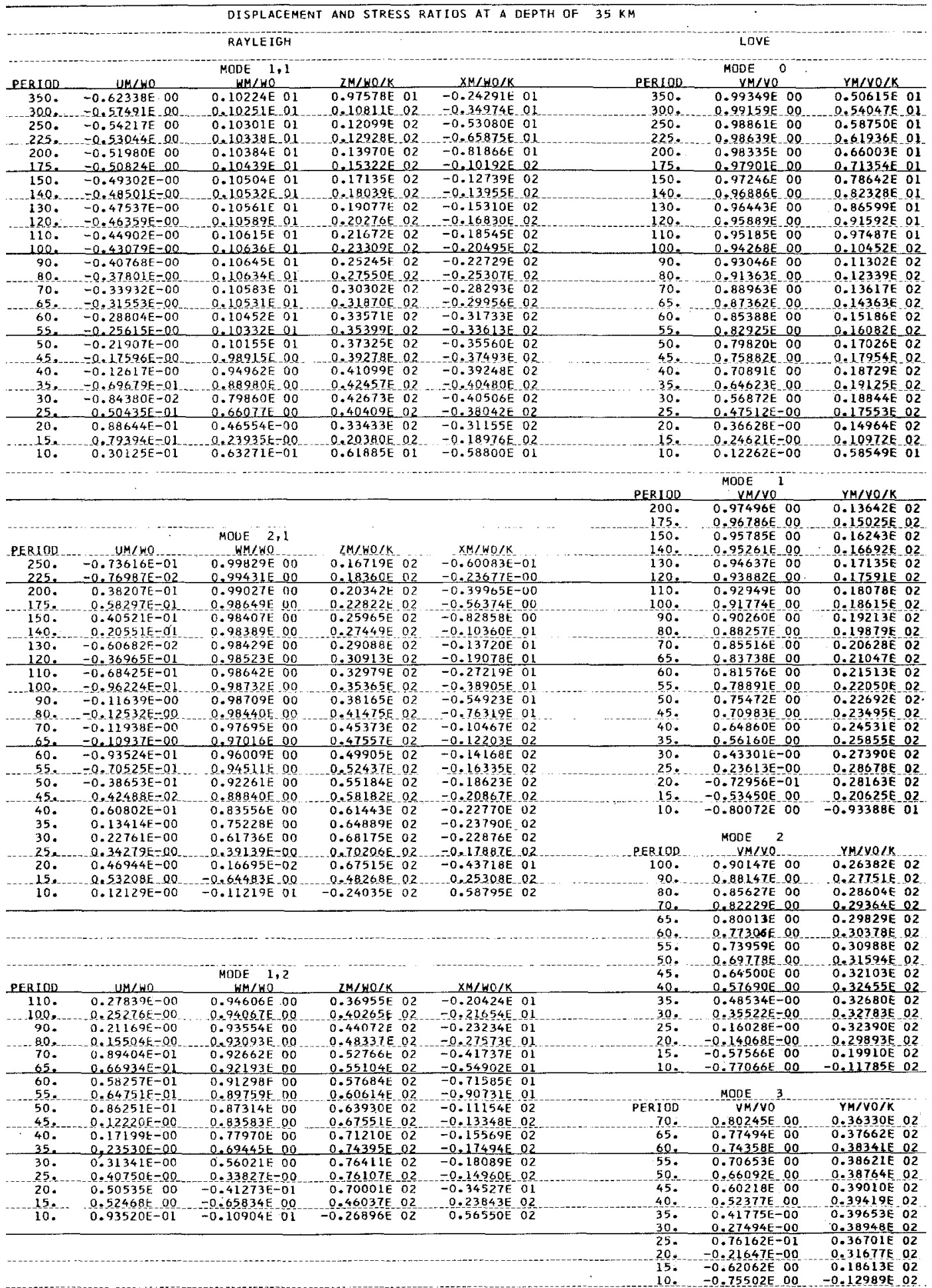


TABLE 12

. OCEAN.

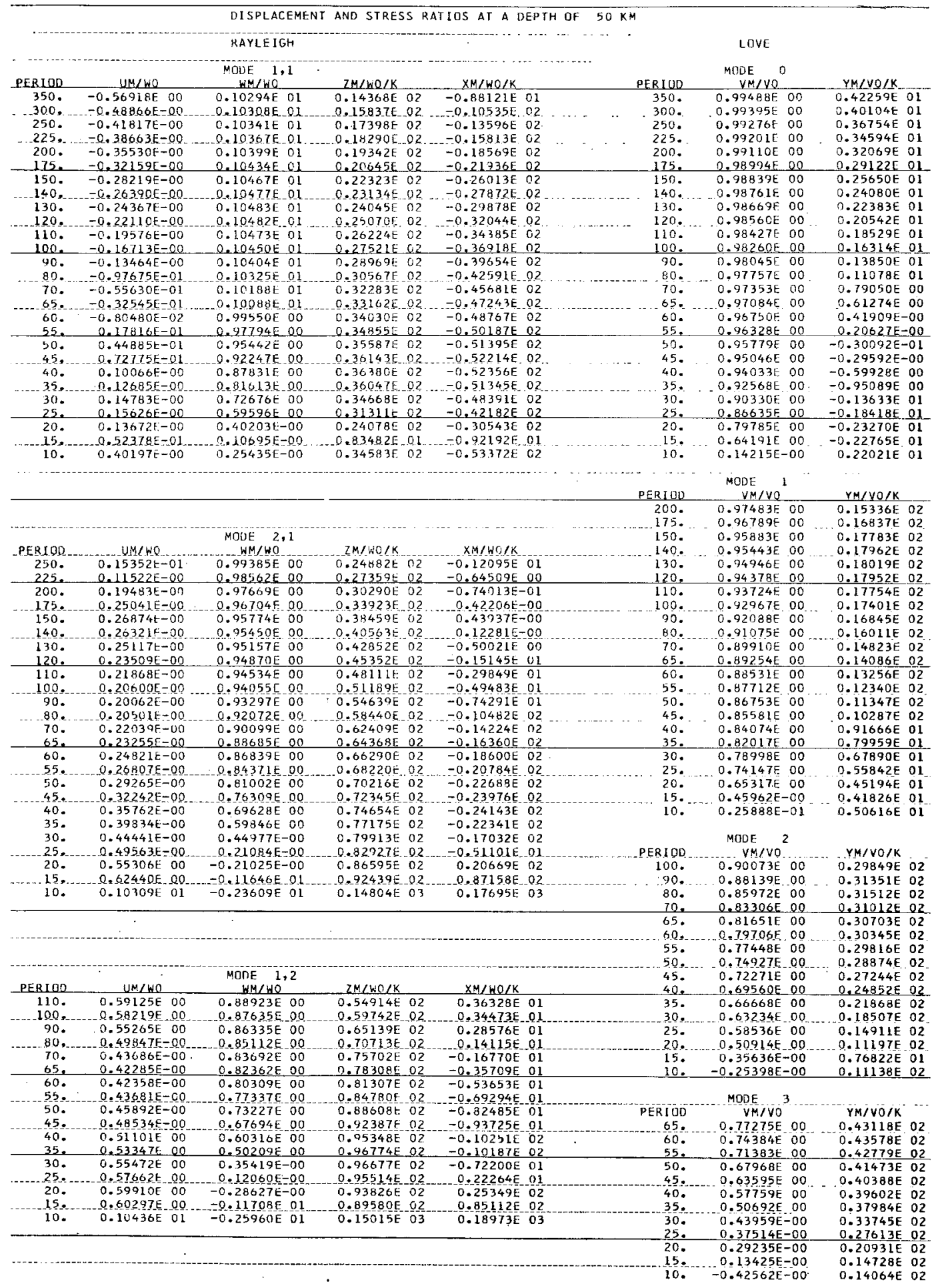


TABLE 13

SHIELD

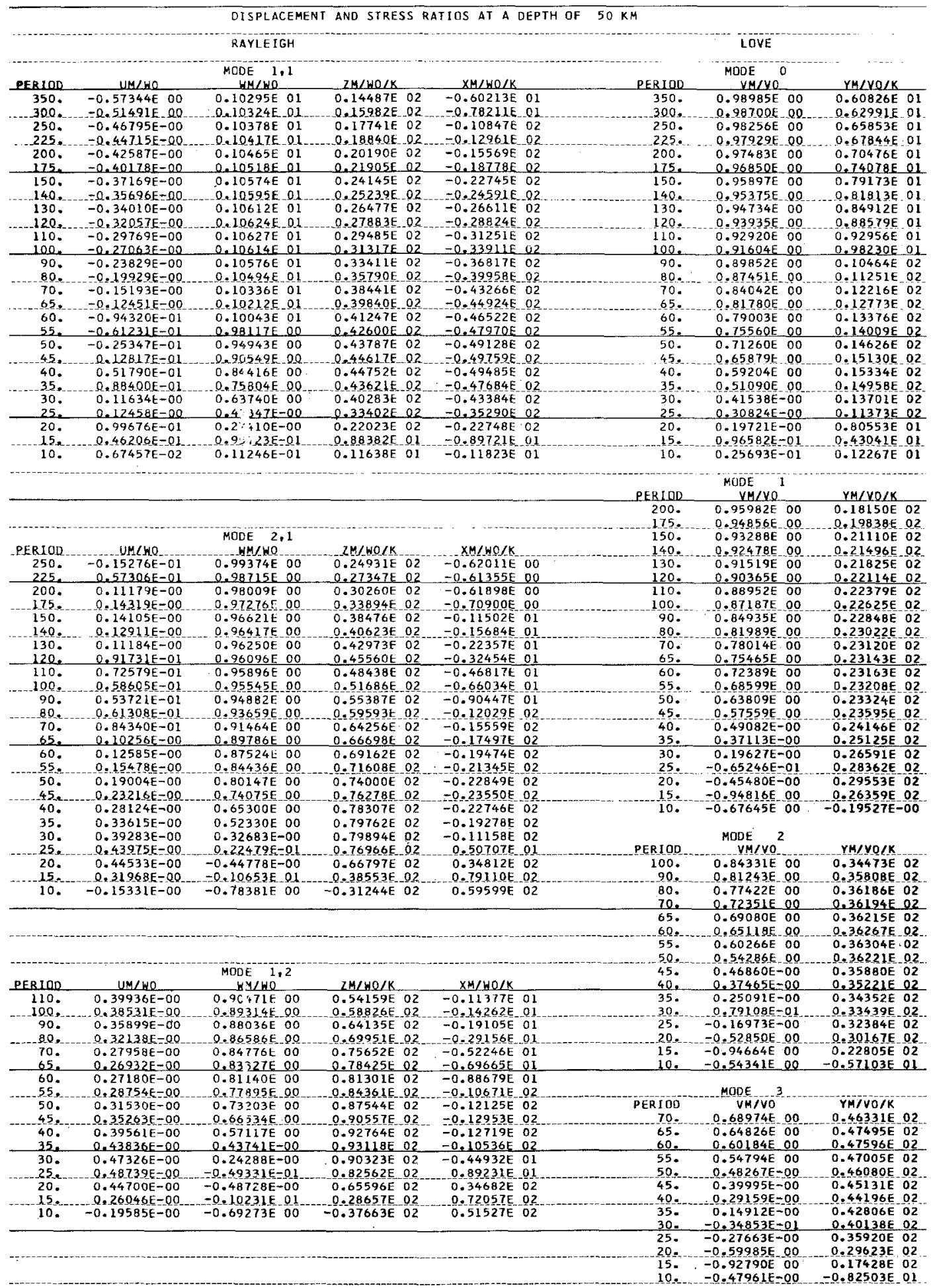


TABLE 14

GCEAIN

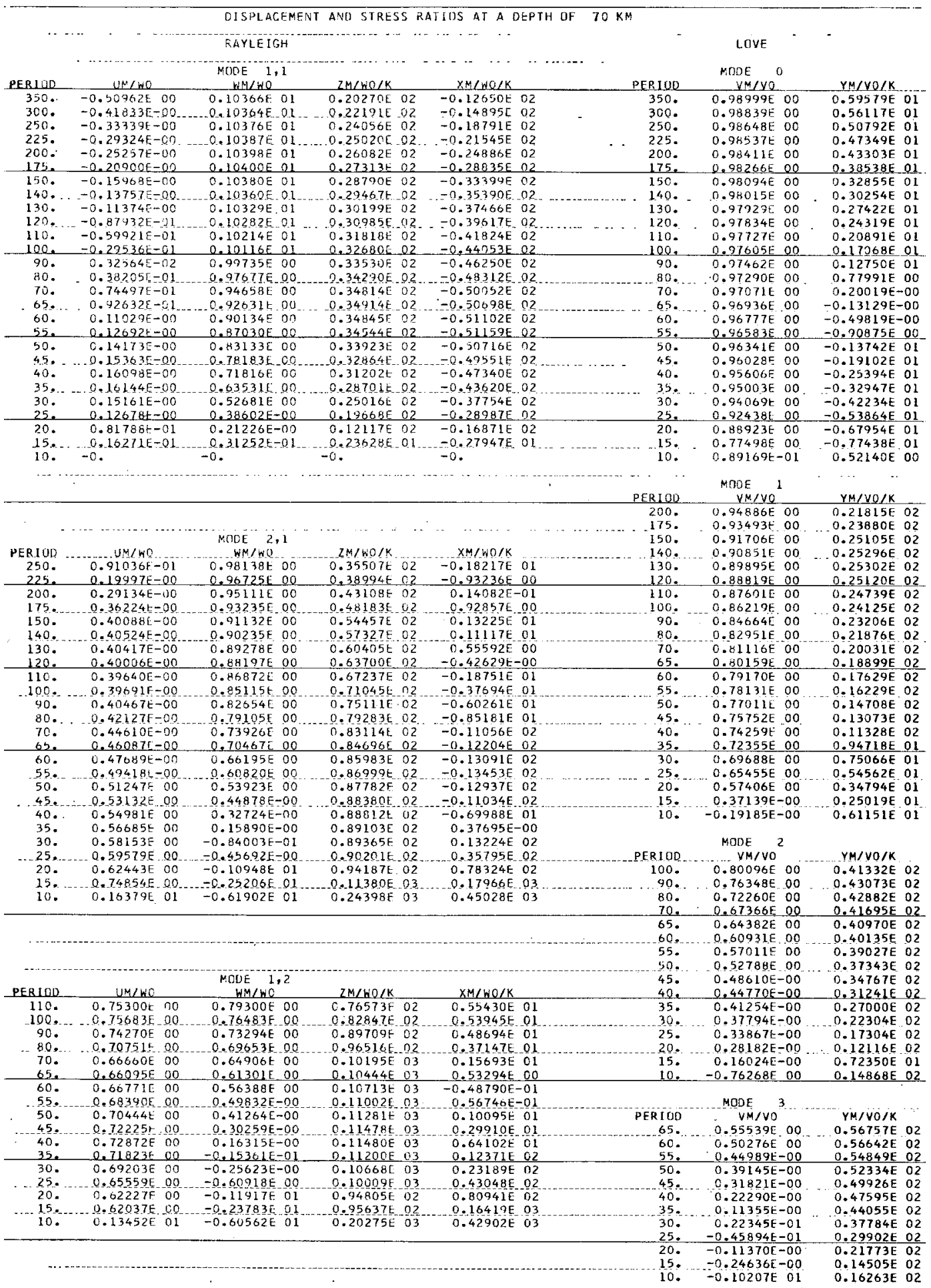


TABLE 15

SHIELD

\begin{abstract}
DISPLACEMENT AND STRESS RATIOS AT A DEPTH OF $70 \mathrm{kM}$
\end{abstract}
RAYLEIGH

LIVE

\begin{tabular}{|c|c|c|c|c|c|}
\hline PER & UM/WO & WM/WO & $2 M / W O / K$ & $x M / w 0 / K$ & \\
\hline 350. & $-0.50996 \mathrm{E}$ OO & $0.10364 \mathrm{E} \mathrm{Ol}$ & $0.20561 \mathrm{E} 02$ & $-0.10533 \mathrm{E} \mathrm{O2}$ & \\
\hline 300. & $=0.43956 \mathrm{E}=00$ & $0.10383 E 01$. & $0.22522 \mathrm{E}-02$ & $=0.13107 E 02$ & \\
\hline 250. & $-0.37666 \mathrm{E}-00$ & $0.10426 E 01$ & $0.24672 \mathrm{E} \quad 02$ & $-0.17359 E \quad 02$ & \\
\hline 225 & $-0.34624 \mathrm{E}-00$ & $0.10455 \mathrm{E} 01$ & $0.25936 E_{-} 02$ & $=0.20271 E 02$ & \\
\hline 200 & $-0.31423 t-00$ & $0.10487 \mathrm{E} 01$ & $0.27428 E \quad 02$ & $-0.23788 \mathrm{E} \quad 02$ & \\
\hline 175. & $-0.27836 E-00$ & $0.10515 \mathrm{E} 01$ & $0.29243 E 02$ & $-0.27996 \mathrm{E} \quad 02$ & \\
\hline 150. & $-0.23566 E-00$ & $0.10527 \mathrm{E} 01$ & $0.31500 E \quad 02$ & $-0.32997 E \quad 02$ & \\
\hline 140. & $=0.21578 \mathrm{E}-00$ & $0.10522 \mathrm{E} \quad 01$ & $0.32559 \mathrm{E} \quad 02$ & $=0.35241 \mathrm{E} 02$ & \\
\hline 130. & $-0.19382 E-00$ & $0.10506 \mathrm{E} \quad 01$ & $0.33723 E \quad 02$ & $-0.37628 \mathrm{E} \quad 02$ & \\
\hline 120. & $-0.16936 \mathrm{E}-00$ & $0.10475 \mathrm{E} \quad 01$ & $0.35000 E 02$ & $=0.40155 \mathrm{E} \quad 02$ & \\
\hline 110. & $-0.14195 \mathrm{t}-00$ & $0.10422 \mathrm{E} \quad 01$ & $0.36397 \mathrm{E} \quad 02$ & $-0.42808 \mathrm{E} \quad 2$ & \\
\hline 100. & $-0.11107 E-00$ & $0.10337 \mathrm{E} Q 1$ & $0.37909 F \quad 02$ & $-0.45552 E 02$ & \\
\hline 90. & $-0.76195 \mathrm{E}-01$ & $0.10201 E 01$ & $0.39514 E \quad 02$ & $-0.48318 \mathrm{E} \quad 02$ & \\
\hline 80. & $-0.36849 \mathrm{E}=0 \mathrm{l}$ & $0.99879 E$ OO & $0.41149 E$ O2 & $-0.50962 E 02$ & \\
\hline 70 . & $0.70843 F-02$ & $0.96509 \mathrm{E} 00$ & $0.42658 \mathrm{E} \quad 02$ & $-0.53204 E 02$ & \\
\hline 65. & $0.30576 \mathrm{E}-01$ & $0.94130 \mathrm{E} 00$ & $0.43269 \mathrm{E} 02$ & $-0.54014 \mathrm{E} \quad 02$ & \\
\hline 60. & $0.54733 \mathrm{E}-0 \mathrm{I}$ & $0.91102 \mathrm{E} 00$ & $0.43692 \mathrm{E} 02$ & $-0.54477 \mathrm{E} \quad 02$ & \\
\hline 55. & $0.79008 E-01$ & $0.87225 E 00$ & $0.43813 E \quad 02$ & $-0.54427 E \quad 02$ & \\
\hline 50. & $0.10241 t:-00$ & $0.82236 E 00$ & $0.43457 \mathrm{E} \quad 02$ & $-0.53635 \mathrm{E} 02$ & \\
\hline 45. & $0.123345-00$ & $0.75779 \mathrm{E} 00$ & $0.42368 \mathrm{E} 02$ & $-0.51765 \mathrm{E} \quad 02$ & \\
\hline 40. & $0.13904 E-00$ & $0.67421 E 00$ & $0.40148 E \quad 02$ & $-0.48370 \mathrm{E} \quad 02$ & \\
\hline 35. & $0.14526 E-00$ & $0.56669 \mathrm{E} 00$ & $0.36245 \mathrm{E} \quad 02$ & $-0.42863 \mathrm{E} \quad 02$ & \\
\hline 30. & $0.13605 t-00$ & $0.43180 E=00$ & $0.29983 E \quad 02$ & $-0.34629 E \quad 02$ & \\
\hline 25. & $0.10556 \mathrm{E}-00$ & $0.27388 \mathrm{E}-00$ & $0.20936 t \quad 02$ & $-0.23495 E \quad 02$ & \\
\hline 20. & $0.56496 \mathrm{~F}-01$ & $0.12001 E-00$ & $0.10267 E \quad 02$ & $-0.11157 \mathrm{E}$ O2 & \\
\hline 15 & $\begin{array}{l}0.13948 \mathrm{E}-01 \\
0.61051 \mathrm{E}-03\end{array}$ & $0.24534 \mathrm{E}-01$ & $0.23653 \mathrm{E} \quad 01$ & $-0.25009 \mathrm{E} 01$ & \\
\hline & IL & ( & $98+10 t-01$ & $10826 \mathrm{E}-00$ & \\
\hline
\end{tabular}

MODE 0

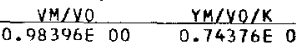
$0.97979 E_{0} 00$. $0.74845 E_{01}$ $0.97344 \mathrm{E}$ OO $\quad 0.75251 \mathrm{E}$ OL $0.96883 \mathrm{E}$ 00 $0.75651 \mathrm{E}$ ol $0.96262 \mathrm{E}$ OO $0.76378 \mathrm{E}$ OL $0.95387 E 00 \quad 0.77662 E$ OL $\begin{array}{ll}0.94084 E \text { OO } & 0.79870 E \text { OI } \\ 0.93374 E .00 & 0.81138 E \text { ol }\end{array}$

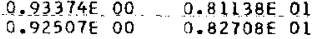
$0.91429 \mathrm{E} 00 \quad 0.84655 \mathrm{E}$ Ol 0.90066 E 00 0.87079E OI

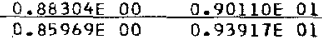
$0.82782 \mathrm{E} \quad 00 \quad 0.98701 \mathrm{E}$ Ol $0.78283 E 00 \quad 0.10465 \mathrm{E} 02$ $0.75316 \mathrm{E}_{0} 00 \quad 0.10806 \mathrm{E} 02$ $0.71692 \mathrm{E} 00 \quad 0.11166 \mathrm{E} \mathrm{O2}$ $0.67236 \mathrm{E}$ 00 $0.11520 \mathrm{E} 02$ $0.61736 \mathrm{E} 00 \quad 0.11813 \mathrm{E}$ O2 $0.54989 \mathrm{E}$.0O $\quad 0.11926 \mathrm{E}$. $0.46887 E-00$ O.11652E O2 $0.27413 \mathrm{E}-00 \mathrm{O}-0.09278 \mathrm{E}$ OL $0.17338 \mathrm{E}-00 \quad 0.63669 \mathrm{E}$ OI $0.17338 \mathrm{E}-00 \quad 0.63669 \mathrm{E}$ OI $0.27737 \mathrm{E}-01 \quad 0.12359 \mathrm{E} 0 \mathrm{I}$ $\begin{array}{ll}0.27737 \mathrm{E}-01 & 0.12359 \mathrm{E} 01 \\ 0.32006 \mathrm{E}-02 & 0.15250 \mathrm{E}-00\end{array}$ MDOE 1

PERLO

200. $0.93301 \mathrm{E} 00 \quad \frac{Y M / Y O / K}{0.24033 \mathrm{E} \mathrm{O2}}$

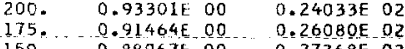
$\begin{array}{lll}175 . \cdots & 0.91464 \mathrm{E} 00 & 0.26080 \mathrm{E} \\ 1502\end{array}$ 140. $0.8770 C E$ OO $\quad 0.27646 E, 02$ 130. $0.86220 \mathrm{E}$ 00 $0.27799 \mathrm{E} 02$

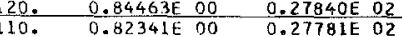

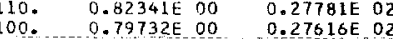
90. $0.76461 \mathrm{E}$ 00 $0.27317 \mathrm{E}$ O2 $80 . \quad 0.72273 \mathrm{E} \quad 00 \quad 0.26822 \mathrm{E} 02$ 70. $0.66761 \mathrm{E} 00$ 0.26064E 02 60. $0.53295 \mathrm{E} \mathrm{OO} \quad 0.25580 \mathrm{E} \mathrm{O2}$ $55 . \quad 0.54130 \mathrm{E} \quad 00 \quad 0.24493 \mathrm{E} .02$ $50 . \quad 0.47812 \mathrm{E}-00 \quad 0.24000 \mathrm{E} 02$ 45. $\quad 0.39583 \mathrm{E}-00 \quad 0.23695 \mathrm{E} 02$ 40. $\quad 0.28366 \mathrm{E}-00 \quad 0.23798 \mathrm{E} 02$ $\begin{array}{rrr}35 . & 0.12357 \mathrm{E}-00 & 0.24608 \mathrm{E} \mathrm{O2} \\ 30 . & -0.11345 \mathrm{E}-00 & 0.26451 \mathrm{E} \mathrm{O2}\end{array}$ $25 .-0.47314 \mathrm{E}-00 \quad 0.29686 \mathrm{E} 02$ $20 .-0.10191 \mathrm{E} 01-0.34707 \mathrm{E}-0.2$ 15. $-0.17203 \mathrm{E} 01 \quad-0.39998 \mathrm{E} 02$ $\begin{array}{ccccc}43 \mathrm{E}-00 & 0.63744 \mathrm{E} 00 & 0.90766 \mathrm{E} 02 & -0.18307 \mathrm{E}-02 \\ 0514 \mathrm{E}-00 & 0.56125 \mathrm{E}-00 & 0.91835 \mathrm{E} 02 & -0.17374 \mathrm{E} & 02\end{array}$

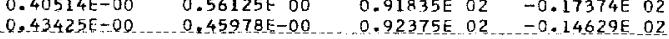
$0.4635 \mathrm{LE}-00 \quad 0.32186 \mathrm{E}-00 \quad 0.92244 \mathrm{E} \mathrm{O2}-0.90300 \mathrm{E} 01$ $0.49045 \mathrm{E}-00 \quad 0.13038 \mathrm{E}-00$ $0.51037 \mathrm{~F}$-0.14093E-0 $\begin{array}{ll}0.51459 \mathrm{E}-00 & -0.52999[-00 \\ 0.48679 \mathrm{E}-00 & -0.10720 \mathrm{E}\end{array}$ $0.91176 \mathrm{E} 02$ $0.88681 \mathrm{E} 02 \quad 0.17320 \mathrm{E} 02$ $0.17320 E 02$
$0.45304 E_{02}$ $0.75126 \mathrm{E} 02$ $0.12420 \mathrm{E} \mathrm{O2}$

20. $0.48679 \mathrm{E}-00 \quad-0.10720 \mathrm{E}$ O1 $0.75126 \mathrm{E}$ O2

$15.38339 \mathrm{E}=00-0.16307 \mathrm{E} 01$
$10 . \quad-0.10280 \mathrm{E}-00 \quad-0.37300 \mathrm{E}-00$ $-0.16618 \mathrm{E} 02$

\title{
$0.86904 E \quad 02$
}

YM/YVO $0.13271 E .03$
$0.30736 \mathrm{E} 02$

YM/YO/K

$0.74282 E 00 \quad 0.44292 \mathrm{E} \mathrm{O2}$

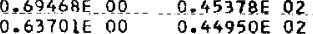
$\frac{0.56256 \mathrm{E} 00}{0.51539 \mathrm{E} 00}-\frac{0.43784 \mathrm{E}}{0.42}$ $0.45898 \mathrm{E}=00 \quad 0.42399 \mathrm{E}-02$ 5. $0.39097 \mathrm{E}-00 \quad 0.41563 \mathrm{E} 02$ 50. $0.30877 \mathrm{~F}-00 \quad 0.40465 \mathrm{E} \quad 02$

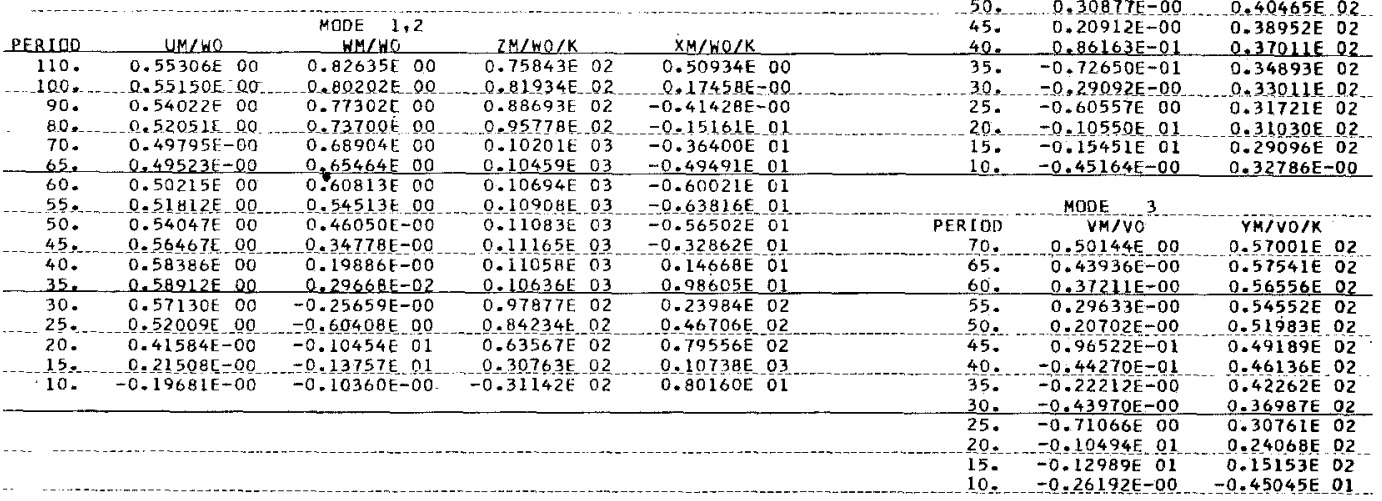


TABLE 16

OCEAN

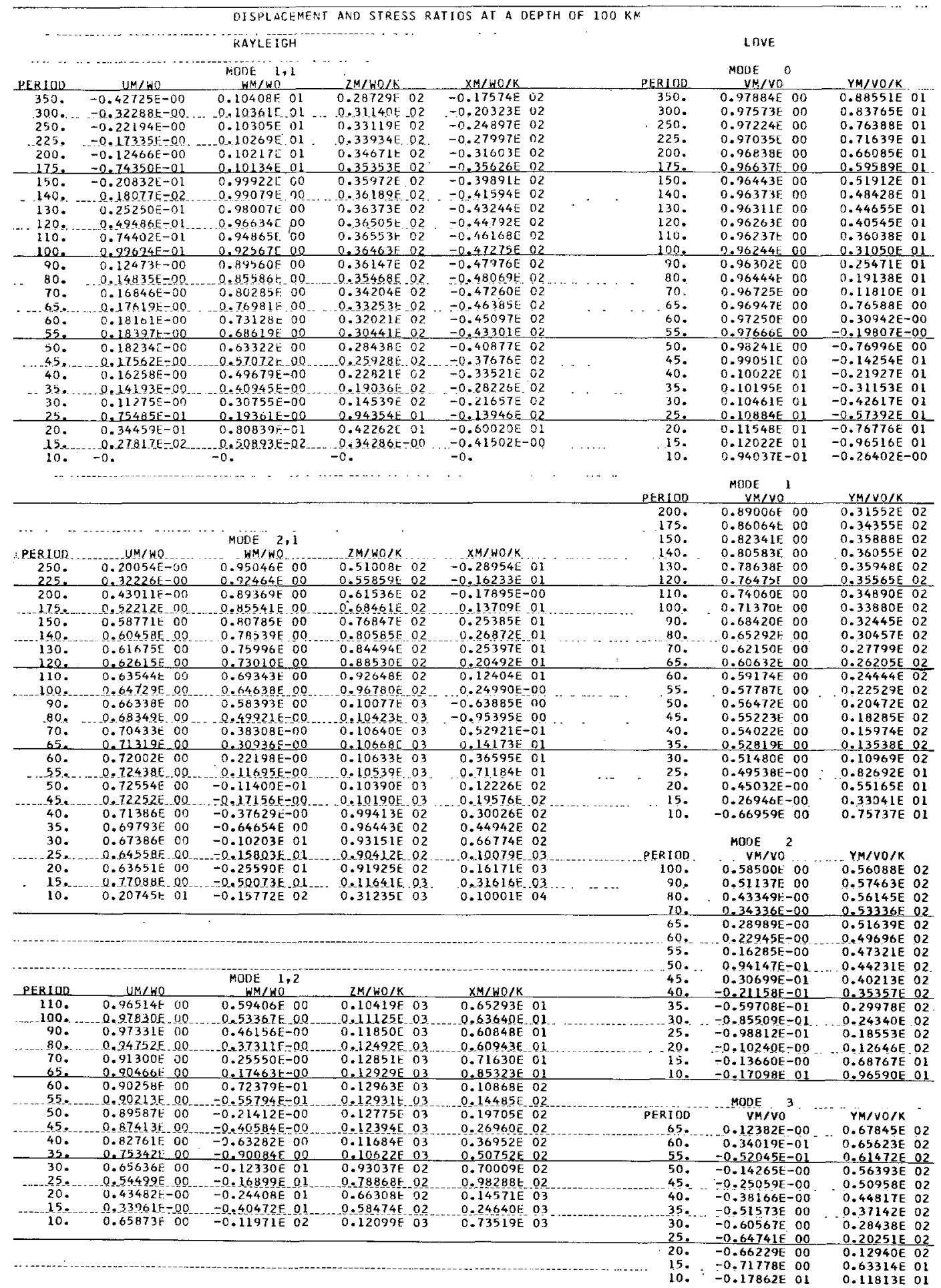


TABLE 17

SHIELD

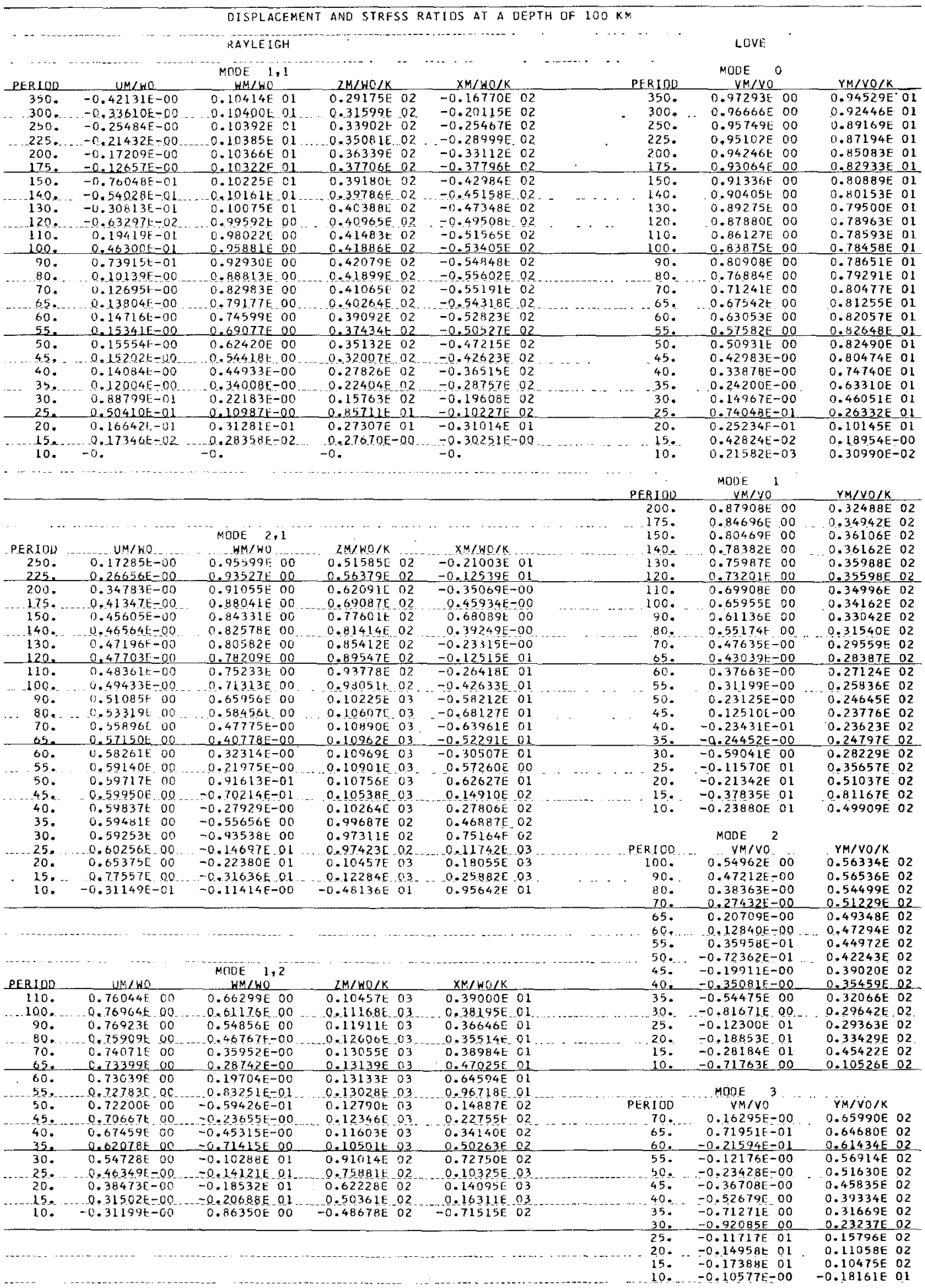


TABLE 18

QCEAN

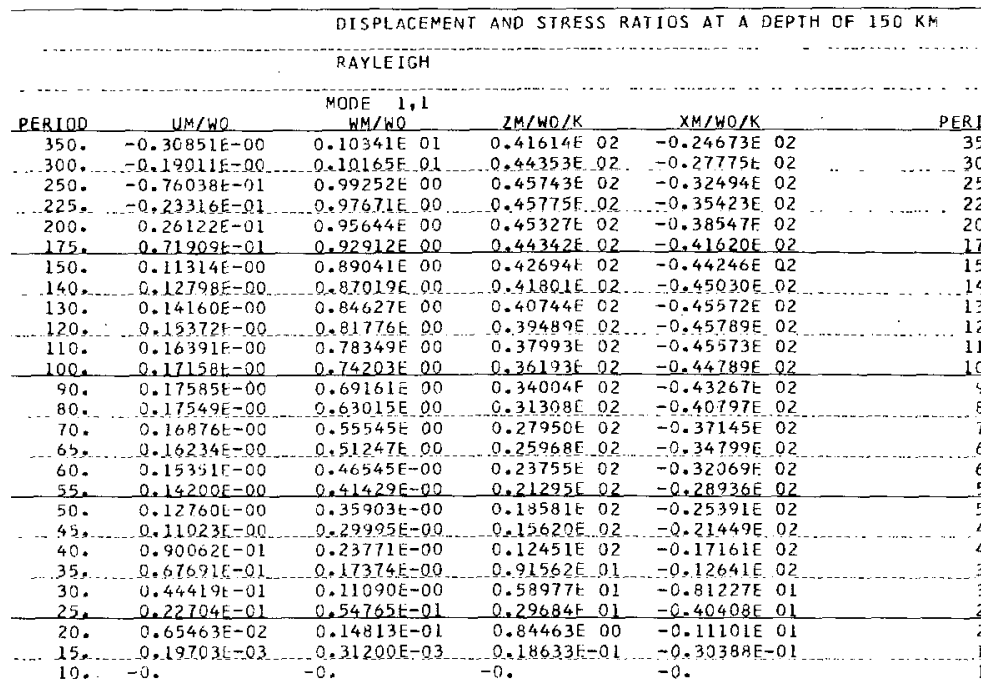

...
100
350.
00.
50.
25.
75.
50.
40.
30.
20.
10.
00.
40.
80.
70.
65.
60.
55.
50.
45.
40.
35.
30.
25.
20.
15.
10.

LOVE $\cdot \cdot \cdot$ VM/YO _ YM/VO/K $0.94807 E 0000.14071 E$ O2 $0.94038 \mathrm{E} 00.0 .13473 \mathrm{E} 02$ $0.12543 E 02$ $0.92698 \mathrm{E} 00-0.11953 \mathrm{E} 02$ $0.92198 \mathrm{EE} 00 \mathrm{0} 0.11275 \mathrm{E} \mathrm{02}$

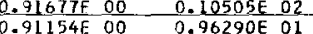
$0.90954 \mathrm{E} 00 \quad 0.92455 \mathrm{E} \mathrm{O1}$ 0.90768 E DO O.88409E OD $0.90605 \mathrm{E} 00 \ldots 0.84137 \mathrm{E} 01$ $0.90480 \mathrm{E}$ OO $0.79623 \mathrm{E}$ OI $0.90414 E 00 \quad 0.74852 E$ OI $0.90444 \mathrm{E} 00.0 .69815 \mathrm{EE}$ OI $0.91074 \mathrm{E} 00 \quad 0.58955 \mathrm{E}$ OI $0.91450 \mathrm{E} 00 \quad 0.56103 \mathrm{E}$ OI $0.91976 E 00^{\circ} 0.53220 \mathrm{E} \mathrm{O1}$ 0.92712E 00 O.50335E 01 $0.93742 \mathrm{E} \mathrm{OO} \quad 0.47493 \mathrm{E} \mathrm{OI}$ $0.95204 E$ EO $0.44774 E$ OI $0.42323 E$ OI 0.105198 O $0.40413 E$ OI 0. $0.11264 \mathrm{E} \quad 01 \quad 0.41104 \mathrm{E} \quad 1$ 0.13460 OI $0.47218 E$ OI $0.75023 \mathrm{E}-01$... $0.56784 \mathrm{E}$ DO

MODE

VM $/$ VO

200. $0.73695 \mathrm{E} 00-\frac{\mathrm{YM} / \mathrm{VO} / \mathrm{K}}{0.46465 \mathrm{E} 02}$

200. $0.73695 \mathrm{E}$ OO $0.46465 \mathrm{E} \mathrm{O2}$

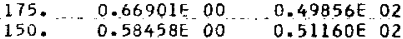

140. $0.54532 \mathrm{E}$ DO $0.50973 \mathrm{E} \mathrm{O2}$ $0.50235 E 00 \quad 0.50359 E$ OZ $0.45515 \mathrm{E}-00 \quad 0.49316 \mathrm{E} .02$ $0.40323 E-00 \quad 0.47829 E 02$ $0.34639 \mathrm{E}-00 \ldots 0.45852 \mathrm{E} .02$ $0.28534 E-00 \quad 0.43299 \mathrm{E} \quad 02$ $0.22225 E-00 \quad 0.40072 E$ O2 $0.16093 \mathrm{E}-00 \quad 0.36122 \mathrm{E} \mathrm{O2}$ $0.13226 E=00 \quad 0.33896 \mathrm{E} \quad 02$ $0.10553 \mathrm{E}-00 \quad 0.31530 \mathrm{E} \mathrm{O2}$ $0.81067 \mathrm{E}-01 \quad 0.29049 \mathrm{E} \quad 02$ $0.59154 \mathrm{E}-01 \quad 0.26480 \mathrm{E}$ O2 $0.40064 \mathrm{E}-01 \quad 0.23843 \mathrm{E}$ O2

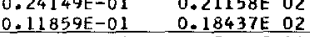
$\frac{0.11859 \mathrm{E}-01}{0.34724 \mathrm{E}-02}-\frac{0.18437 \mathrm{E} \mathrm{O2}}{0.15687 \mathrm{E} \mathrm{O2}}$ $-0.24719 \mathrm{E}-02 \quad 0.12892 \mathrm{E} 02$ $\begin{array}{ll}0.24719 \mathrm{E}-02 & 0.12892 \mathrm{E} \\ -02 & 02\end{array}$ $-0.17142 \mathrm{E}-01 \quad 0.98943 \mathrm{E} 01$ $-0.86546 E$ DO $-0.45879 E$ OI MOOE 2 VMIVO Y YM/YO/K

$0.86459 E-01$ 0.68616E 02 $-0.51723 E-01 \quad 0.66614 E$ O2 $-0.18887 \mathrm{E}-00 \quad 0.61152 \mathrm{E} 02$ $-0.33519 E-00 \quad 0.53506 E \quad 02$ $-0.41538 \mathrm{E}-00 \quad 0.49010 \mathrm{E} 02$ $-0.49974 \mathrm{E}-00 \quad 0.43973 \mathrm{E} \mathrm{O2}$ -0.58430 E OO $0.38313 E$ O2 $-0.66088 \mathrm{E} 00 \quad 0.32105 \mathrm{E} 02$ $-0.71900 \mathrm{E}$ OO $0.25724 \mathrm{E} 02$ $-0.75175 E$ OO $0.19689 E$ O2

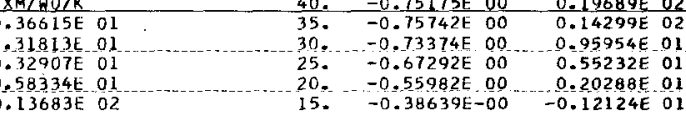

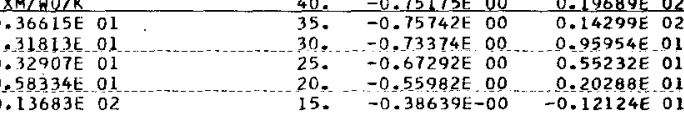

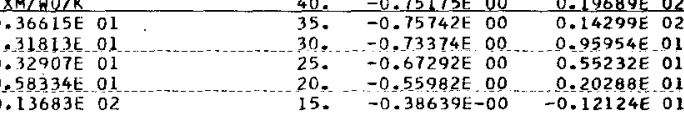

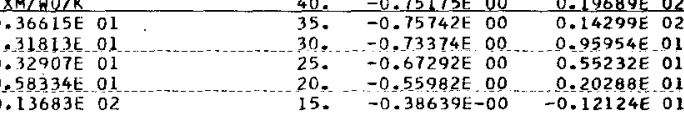

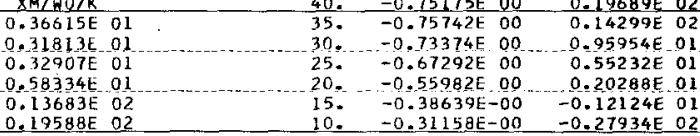
$0.19588 \mathrm{E} \quad 02$ $0.26779 \mathrm{E} 02$ $0.35413 E-02$ PERIDD VM/VO YMONK

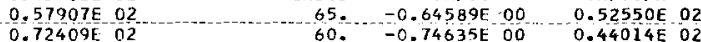
$0.89306 \mathrm{~F} 02$ 55. $-0.82620 \mathrm{E}$ OD $-0.34553 \mathrm{E}, 02$ $45 .-0.94245 \mathrm{E} \quad 00 \quad 0.13599 \mathrm{E} .02$ O.11520E OI $35 .-0.91387 \mathrm{E}$ 00 $-0.0 .0932 \mathrm{E} 02$ 30. $-0.80549 \mathrm{E}$ 00 -0.17976E O2 25. $-\frac{-0.66309 E 00 \quad-0.19607 E 02}{-0.47523 E-00}$ 20. $-0.47523 E-00 \quad-0.18649 E$ O2

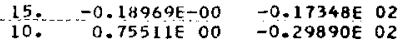


TABLE 19

SHIELD

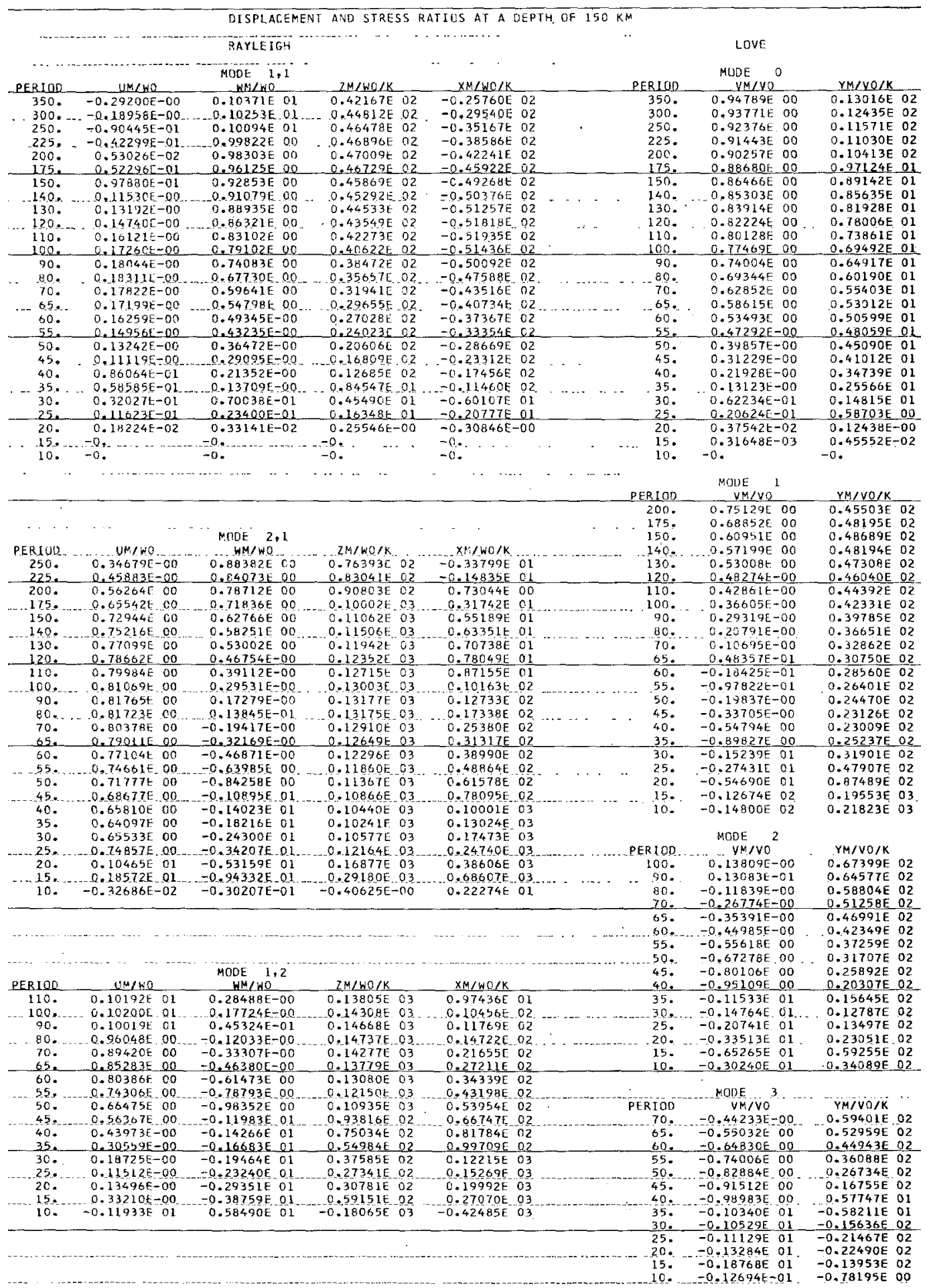


TABLE 20

OCEAN

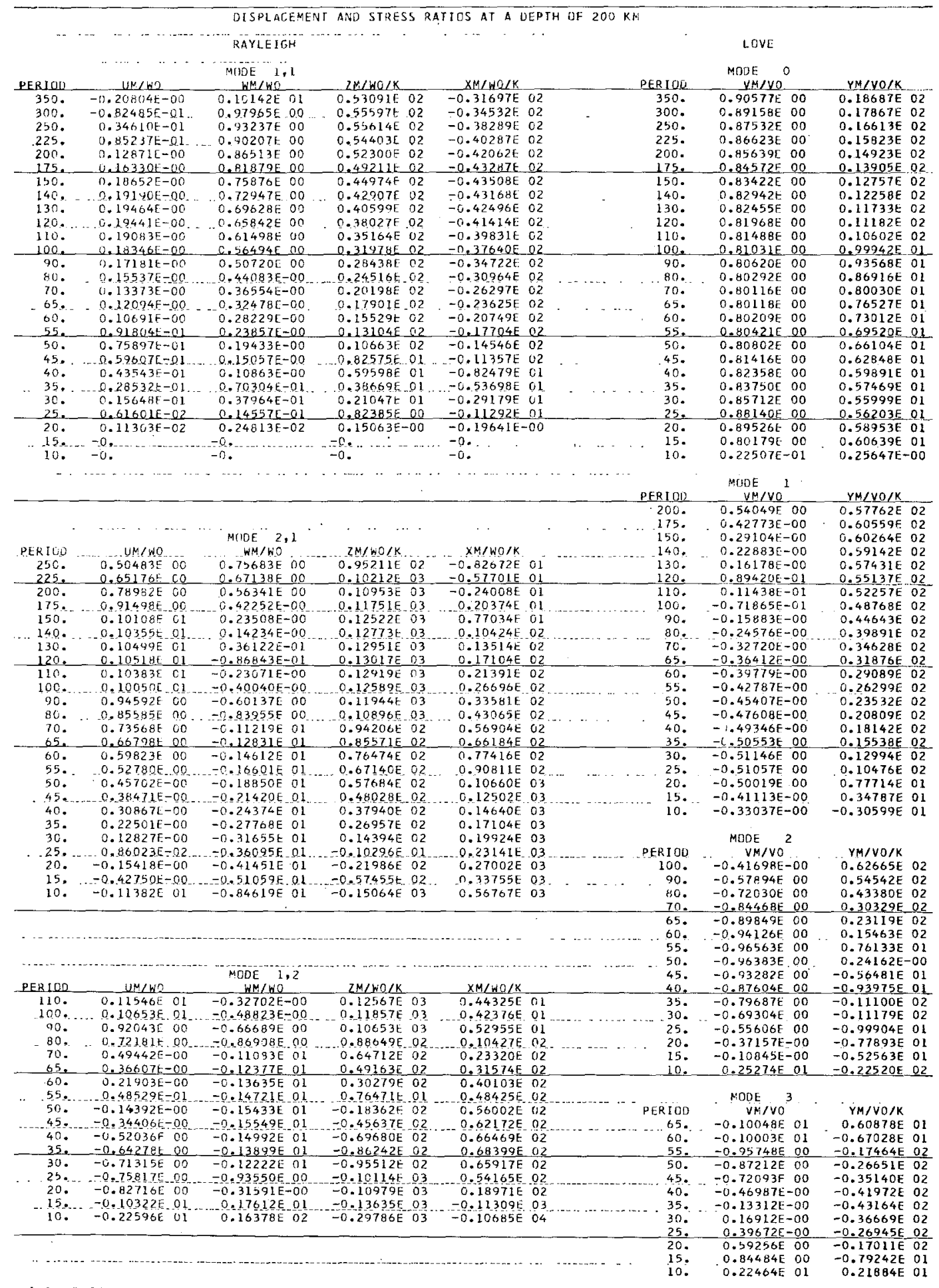


TABLE 21

SHLELD

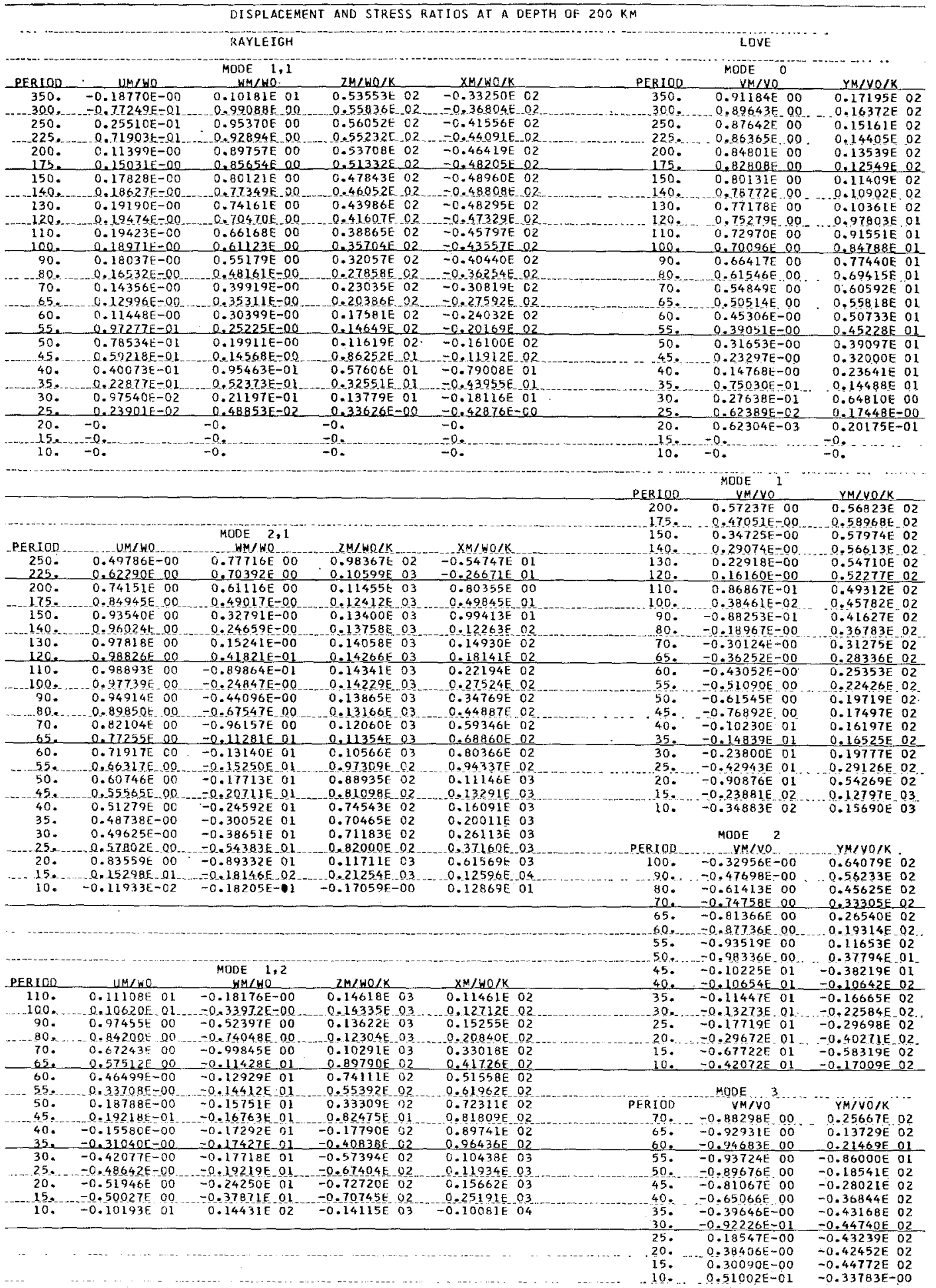


TABLE 22

DCEAN

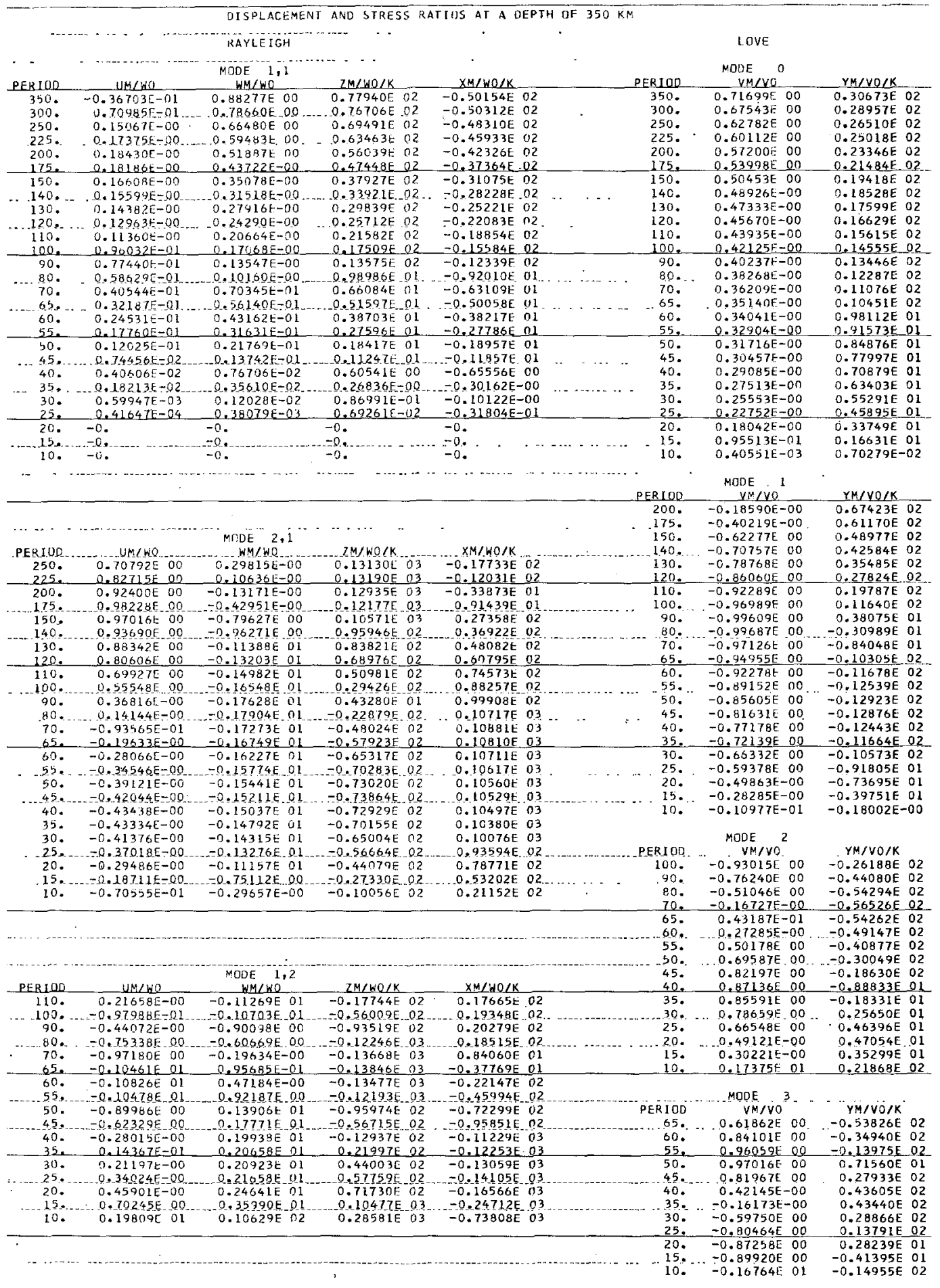


TABLE 23

SHIELD

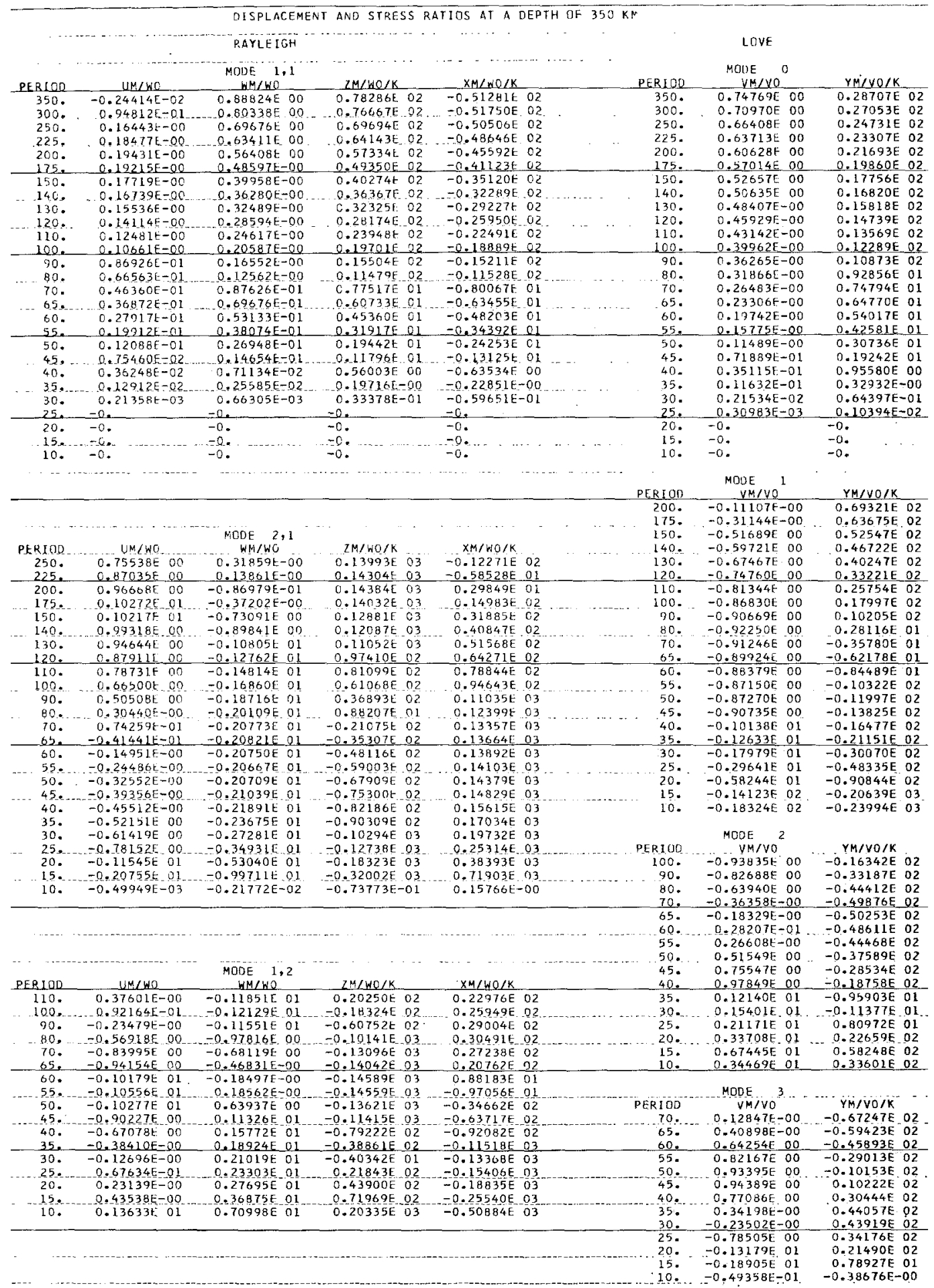


TABLE 24

DCEAN

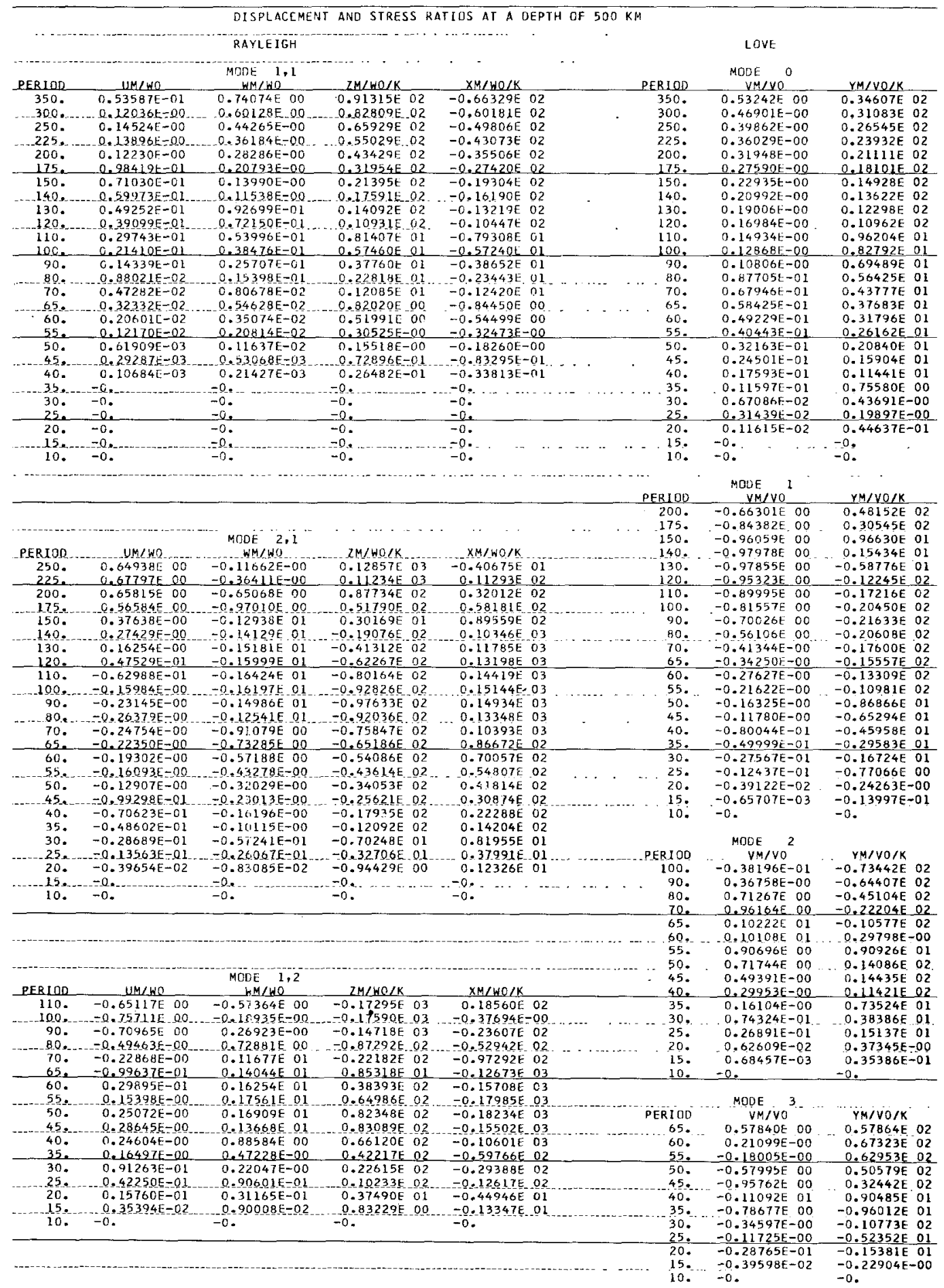




\section{TABLE 25}

SHIELD

OISPLACEMENT AND STRESS RATIDS AT A DFPTH OF $500 \mathrm{KM}$

RAYLEIGH

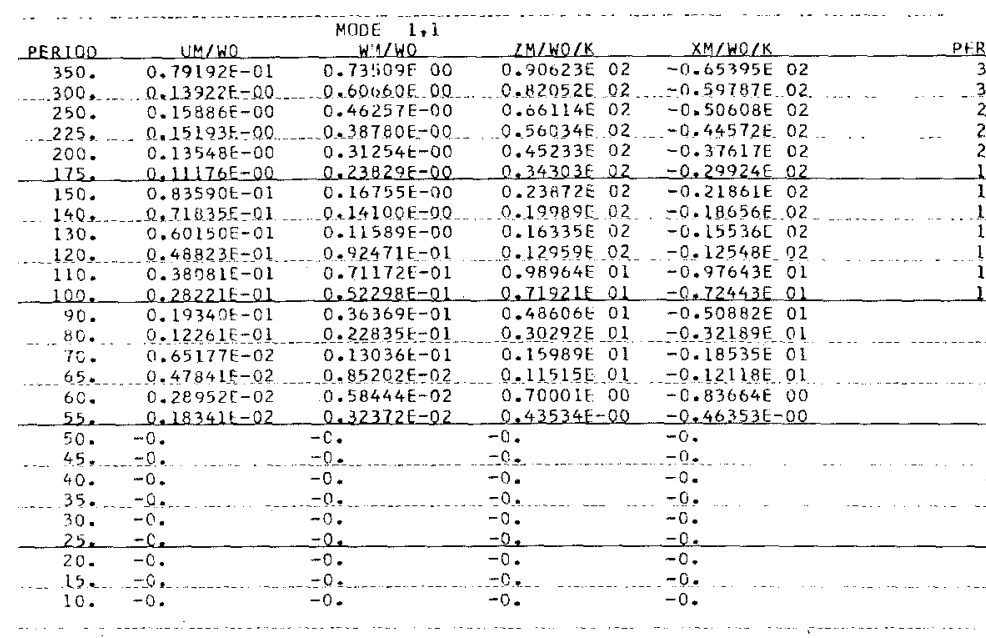

$Y M / V O$ $0.56804 \mathrm{E} 00-0.33915 \mathrm{E} \mathrm{O2}$ $0.50932 E_{0} 00$. $0.30594 E_{02} 02$ $0.44210 E-00 \quad 0.26514 \mathrm{E} 02$ $0.40426 \mathrm{E}-00 \ldots 0.24069 \mathrm{E} \mathrm{O2}$ $0.36278 E-00$ O. $0.21385 E$ O2 $0.31683 \mathrm{E}-0 \mathrm{O} \quad 0.18455 \mathrm{E} 02$ $0.26550 \mathrm{E}-00 \mathrm{0}-15268 \mathrm{E} 02$ $0.24327 E-00$ 0.13919E D2 $\begin{array}{ll}0.24327 E-00 & 0.13919 E .02 \\ 0.22000 E-00 & 0.12528 E\end{array}$ $0.19569 E-00$ 0.12528E 02 $0.19569 E-00 \quad 0.11094 \mathrm{E}$.2 $0.14406 \mathrm{E}-00 \quad 0.81112 \mathrm{E} O 1$ $0.11700 E-00 \quad 0.65744 E$ OL $0.0968-01, \quad 0.50264 E$ OI $0.62170 \mathrm{E}-01$ 0.34985E OL $0.48943 E-01 \quad 0.27504 E$ OL $0.36337 \mathrm{E}-01 \quad 0.20568 \mathrm{E}$ 01 $0.24751 \mathrm{E}-01 \quad 0.14084 \mathrm{E} \quad 0 \mathrm{~L}$ $0.14766 \mathrm{E}-01 \quad 0.84728 \mathrm{E} 00$ $0.71259 E-02 \quad 0.41430 E-00$ $0.24399 E-02 \quad 0.14471 E-00$ $0.52016 \mathrm{E}-03 \quad 0.27576 \mathrm{E}-01$ $-0$.

$-0$.

$\begin{aligned} & -0 . \\ & -0 .\end{aligned}-0$.

MODE

$-0.62447 \mathrm{E} 00$

$\begin{array}{ll}200 . & -0.62447 \mathrm{E} \\ 175 . \quad-0.80335 \mathrm{~F} & 00\end{array}$

150. $-0.92700 E$ OO

140. -... - - $0.95323 \mathrm{E} 00$

120. $-0.95074 \mathrm{E} 00$

$100.0 .91461 \mathrm{E} 00$

$100 . \quad-0.84948 \mathrm{E} \quad 00$

$00.0 .0 .02310 \mathrm{E}$

$80 .-0.62490 E-00$

70. $-0.47826 \mathrm{E}-00$

$65=-0.40401 \mathrm{E}-00$

60. $-0.33331 \mathrm{E}-00$

$-0.26923 \mathrm{E}-0.0$

$-0.21458 \mathrm{E}-00$

$-0.17163 E-00$

$-0.14167 \mathrm{E}-00$

$-0.12389 E-00$

$-0.11455 \mathrm{E}-00$

$-0.10748 \mathrm{E}-00$

$-0.94075 E-01$

$.12723 E \quad 03$

$0.91979 \mathrm{E}$. 2

$0.75006 \mathrm{E} 02$

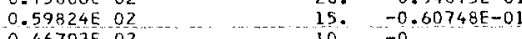

$0.46793 \mathrm{E}$ O2

$0.35918 \mathrm{E}$ O2

MODE 2

$100 .-0.13522 E-00 \quad-0.71181 E 02$

$0.13361 \mathrm{E} 02$

0 .

YMAVO/X

$.51517 \in 02$

$0.34945 \mathrm{E} 02$

$0.15255 \varepsilon$ Q

$0.74757 E$ O

$-0.63086 \mathrm{E} 01$

$0.11764 \mathrm{E} 02$

$0.15864 E_{0} 02$

-

$-0.15417 \mathrm{TE}$ O2

$-0.13616 \mathrm{E} 02$

$-0.11692 \mathrm{E} \quad 02$

$0.98337 \mathrm{E}$ OI

$-0.82340 \mathrm{E}$ OI

$-0.70527 \mathrm{E}$ ol

$-0.63411 \mathrm{E}$ 01

-0.56862 E 01

$-0.50097 \mathrm{E}$ OI

$-0.32874 \mathrm{E} 01$

$\sim 0$

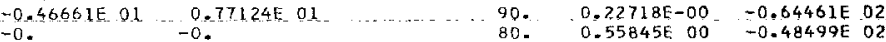

0.55B45E OO

$0.83781 \mathrm{E} 00 \quad-0.28182 \mathrm{E} .02$

$0.94172 E$ OO $-0.17277 E$ D2

$0.10009 \mathrm{E} 01 \quad=0.63944 \mathrm{E}$ OI

$0.99316 \mathrm{E}$ OO $0.36358 \mathrm{E}$ OI

$0.74480 \mathrm{E} 00 \quad 0.15775 \mathrm{E} \mathrm{O2}$

$0.56196 \mathrm{E} 00 \quad 0.16295 \mathrm{E} 02$

$0.40120 \mathrm{E}-00$

$0.27984 E-00 \quad 0.11501 \mathrm{E}-02$

$0.86564 \mathrm{E}$ O1

$0.12306 \mathrm{E}=00 \quad-0.59292 \mathrm{E}-01$

\begin{tabular}{ll}
$0.60884 \mathrm{E}-01$ & $0.30602 \mathrm{E}$ \\
$0.31415 \mathrm{E}-02$ & $0.50178 \mathrm{E}-01$ \\
\hline
\end{tabular}

$\begin{array}{ll}0.18067 \mathrm{E} 03 & 0.14702 \mathrm{E} 02 \\ -0.17423 \mathrm{E} 03 & -0.59255 \mathrm{E} \text { ol }\end{array}$

$-0.13477 \mathrm{E}$ 03 $-0.34591 \mathrm{E} 02$

$\begin{array}{llllll}0.10174 \mathrm{E} & 01 & -0.74355 \mathrm{E} & 02 & -0.75802 \mathrm{E} & 02 \\ 0.12745 \mathrm{E} & 01 & -0.42544 \mathrm{E} & 02 & -0.10286 \mathrm{E} & 03 \\ 0.15335 \mathrm{E} & 01 & -0.10390 \mathrm{E} & 02 & -0.13318 \mathrm{E} & 03\end{array}$

$\begin{array}{llllll}0.10174 \mathrm{E} & 01 & -0.74355 \mathrm{E} & 02 & -0.75802 \mathrm{E} & 02 \\ 0.12745 \mathrm{E} & 01 & -0.42544 \mathrm{E} & 02 & -0.10286 \mathrm{E} & 03 \\ 0.15335 \mathrm{E} & 01 & -0.10390 \mathrm{E} & 02 & -0.13318 \mathrm{E} & 03\end{array}$

$65 .-1.0 .323375-00$

60. $-0.17950 E-00$

5. -0.31

$0.11276 \mathrm{E}-0000.18806 \mathrm{E}$ Ol

$\begin{array}{lll}0.21594 \mathrm{E} 02 & -0.16279 \mathrm{E} 03 & \\ 0.50712 \mathrm{E} 02 & -0.18347 \mathrm{~F} 03 & \text { PERIOO }\end{array}$

$0.71013 \mathrm{~F} \quad 02 \quad-0.18367 \mathrm{E} 03$

MODE 3

VMIVO

$0.85387 E 00$
$0.67543 \mathrm{E} 00$

$0.67543 \mathrm{E} 00$

YMIYOIK

$0.24493 \mathrm{E} \quad 02$ $0.75301 t$ O2 $-0.15596 E$ O

$0.97336 \mathrm{E} 00 \quad 0.62530 \mathrm{E} 02-0.10929 \mathrm{E} \quad 03$

0.55309600

$0.28 .48 \mathrm{E}-00$

$0.42208 \mathrm{E} 02 \quad-0.65062 \mathrm{E} \mathrm{O2}$

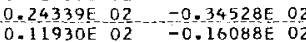

$0.40515 \mathrm{~F}$ ol

-0 .
$0.72132 \mathrm{E}-01$

$0.30494 \mathrm{E}-0 \mathrm{O}$

$-0.71178 \mathrm{E}$ oO

$-0.10513 \mathrm{E}-01$

$-0.10896 \mathrm{E}$ Ol

$-0.77506 \mathrm{E} 00$

$-0.18973 \mathrm{E}-00$

$-0.54904 \mathrm{E}-01$

$-0$.
$0.47701 E$ O2

$0.59306 \mathrm{E} \mathrm{O2}$

$0.53504 E .02$

$0.39878 \mathrm{BE} \mathrm{D2}$

$0.19722 E 02$

$-0.26297 \mathrm{E}$ O1

$-0.14565 \mathrm{E}$ 02

$-0.75303 \mathrm{E}$ ol

$-0.24912 \mathrm{E} 01$ 
TABLE 26

DCEAN.

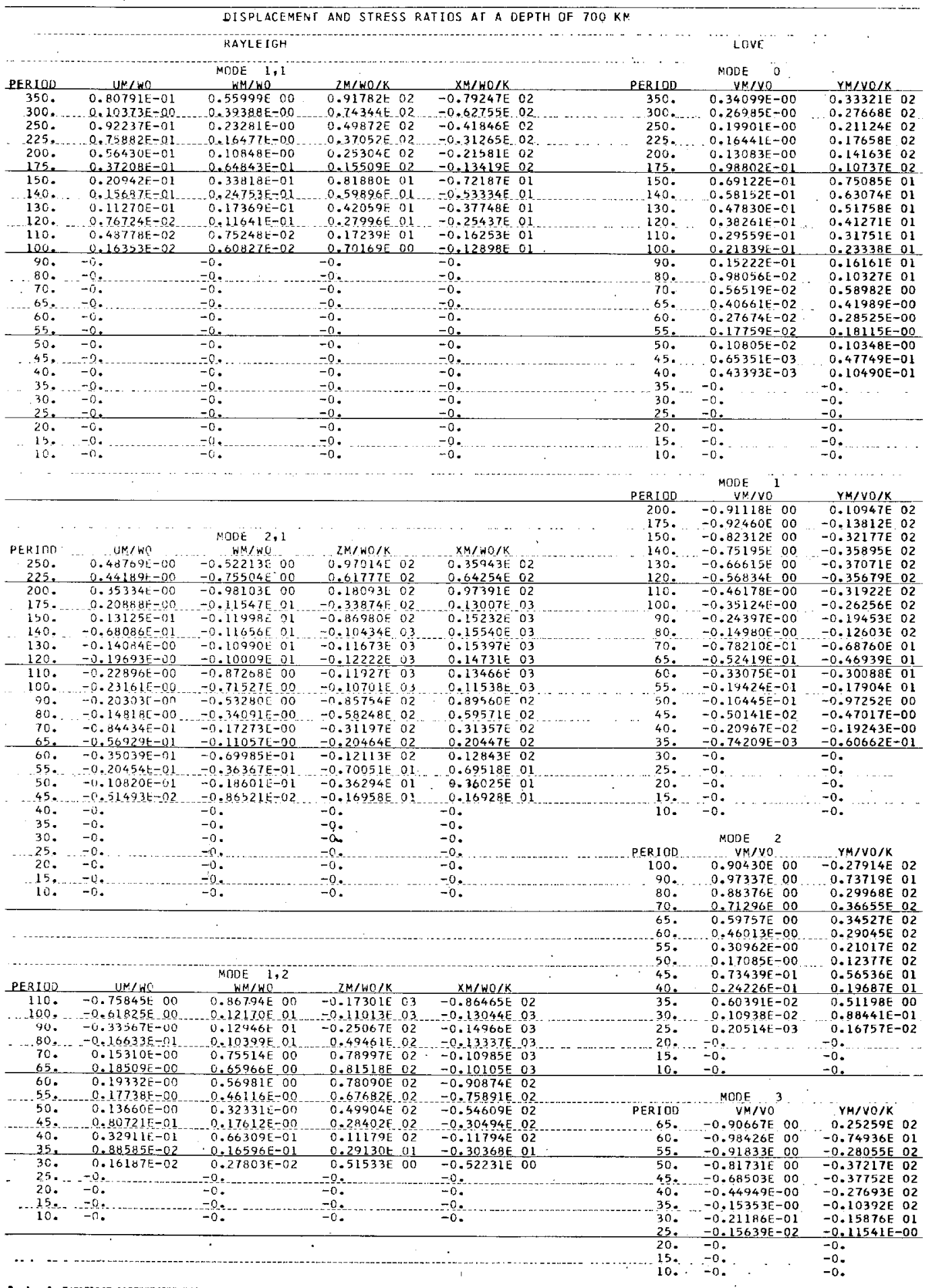


TABLE 27

SHIELD

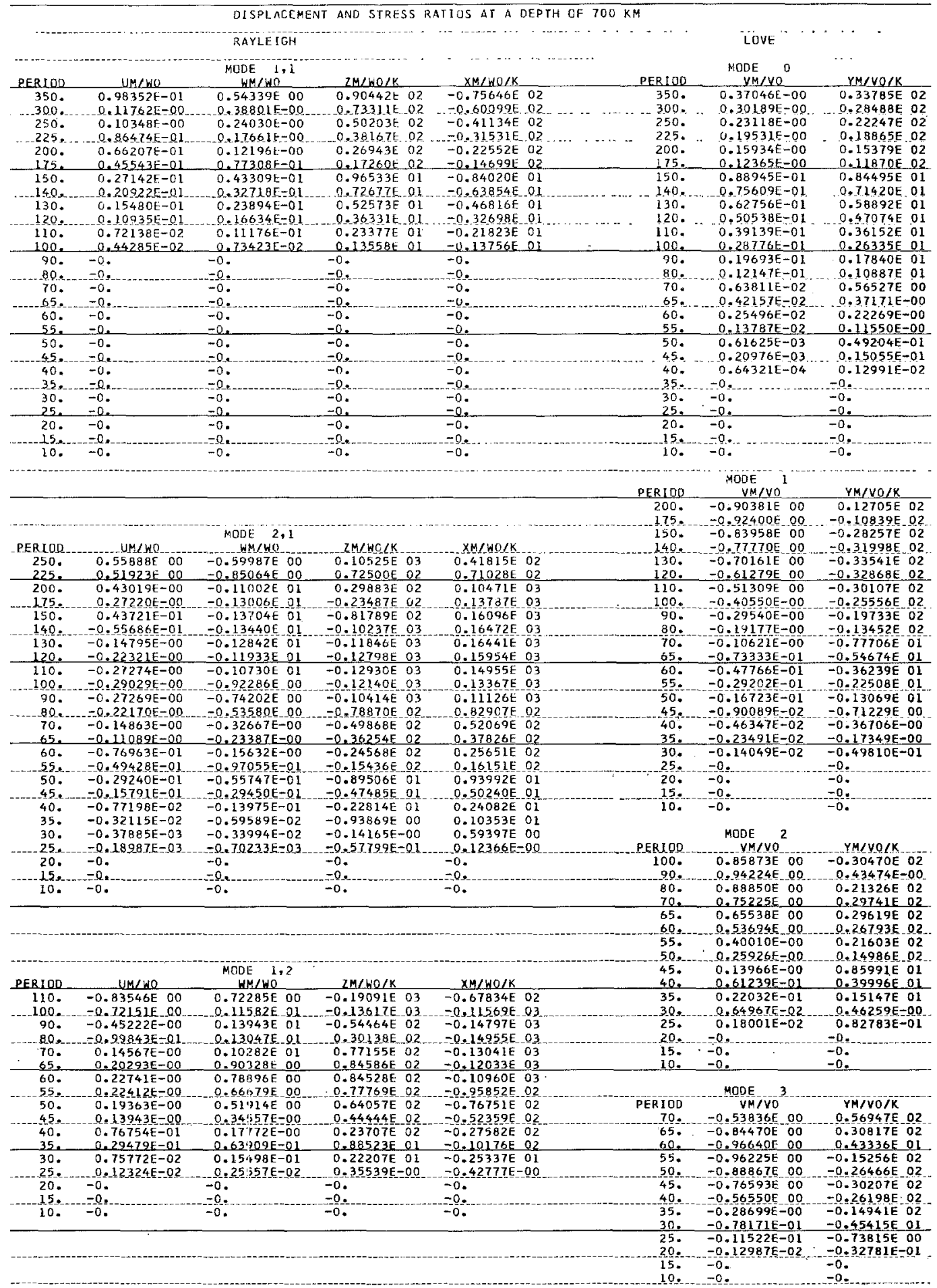


of first higher mode to fundamental mode are displayed in Figures 9 and 10 for Rayleigh and Love waves, respectively. Figure 11 shows the ratio of the second higher mode to the fundamental Rayleigh mode. The ratio zeros and infinities for this doublecouple orientation are determined by the nodal periods in horizontal displacement at the source depth. Thus the spectra of each higher mode has one more zero than the next lower mode. For a given mode the displacement zeros migrate downward in depth with increasing period. Therefore successively deeper sources have spectral zeros at successively longer periods.

Even though all the spectral ratios shown in Figures 8 to 11 have nodes and infinities which are sensitive to the source depth, the most promising is the fundamental Rayleigh to Love ratio. The easily identified large amplitudes of fundamental surface waves in the time domain are important to taking meaningful spectral ratios. The interference of similar signals can cause spectral holes which, along with spectral zeros due to source time history and finiteness, make identification of the source depth mini

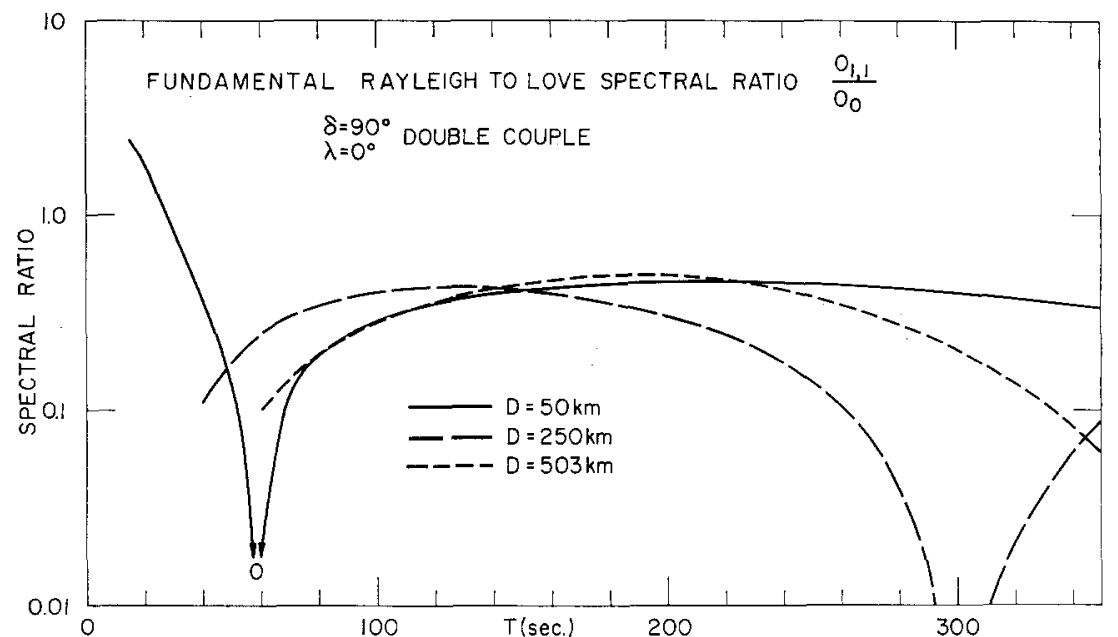

Fig. 8. Spectral ratio (Rayleigh fundamental-mode/Love fundamental-mode) for a double couple at several values of depth in an oceanic model.

mum improbable. If there is enough energy in the observed fundamental Love wave, the minimums in Love spectra will be due to interferences, such as multipath arrivals, and source time and finiteness functions. Thus ratios should not be formed at periods for which there is a lower power level in the Love-wave spectrum.

The ratio minima are dependent on the fault orientation parameters of dip, $\delta$ and slip, $\lambda$, as well as source depth. The ratio of fundamental Rayleigh to Love has a true node at all azimuths only for fault models, $(\delta, \lambda)$ of $\left(90^{\circ}, 0^{\circ}\right)$ and $\left(90^{\circ}, 180^{\circ}\right)$. The $\left(45^{\circ}, 90^{\circ}\right)$ and $\left(45^{\circ}, 270^{\circ}\right)$ faults can have true nodes at azimuthal angles from the strike of $\theta=45^{\circ}, 135^{\circ}, 225^{\circ}$, and $315^{\circ}$.

The nodal period as a function of source depth for the vertical pure strike-slip fault model, $\left(90^{\circ}, 0^{\circ}\right)$, is shown in Figure 12. For the oceanic and shield Earth structures, the relation between period and source depth is almost linear down to depths of $150 \mathrm{~km}$. A rough estimate of the source depth can be obtained by equating the depth in kilometers to the nodal period in seconds. For a homogeneous Poisson solid half-space, the relation between source depth, $h$, and the nodal period is given by

$$
h=(0.19) \times(C T)
$$


(Ben-Menahem and Toksöz, 1963). The ratio of source depth to this critical wavelength, $\lambda_{c}=C T$, versus source depth is shown in Figure 13 for the two models.

Figure 14 illustrates the effects of varying the fault geometry parameters $(\delta, \lambda)$. For changes in dip and slip of less than $10^{\circ}$ from the vertical pure strike-slip fault $\left(90^{\circ}, 0^{\circ}\right)$. the minimum near 60 sec is recognizable and essentially stable. However, at an observer azimuth of $\theta=22.5^{\circ}$ a change of $15^{\circ}$ in $\delta$ or $\lambda$ can virtually eliminate the mini-

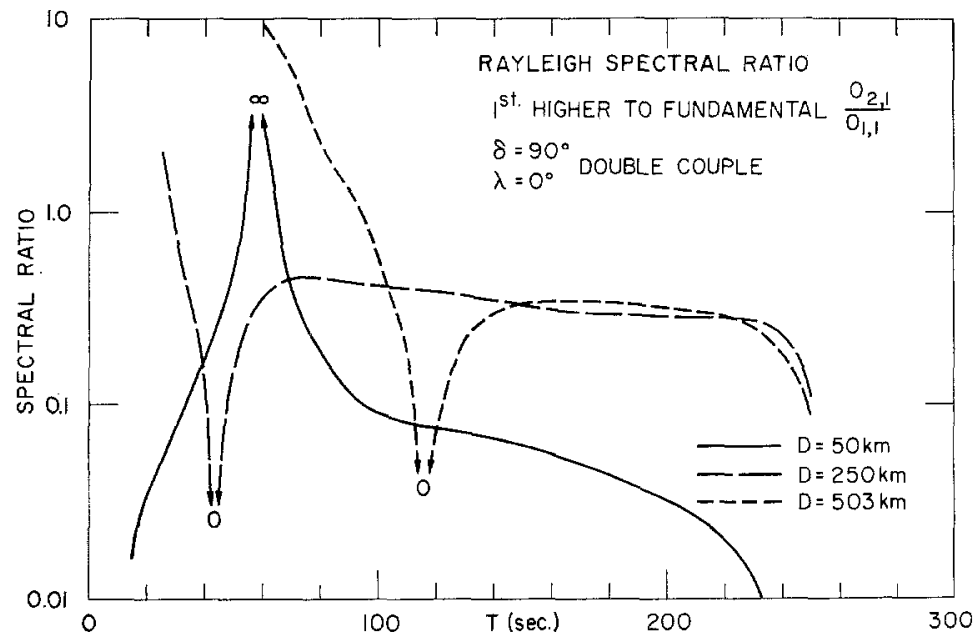

FIG. 9. Spectral ratio (Rayleigh first-mode/Rayleigh fundamental-mode) for a double couple at several values of depth in an oceanic model.

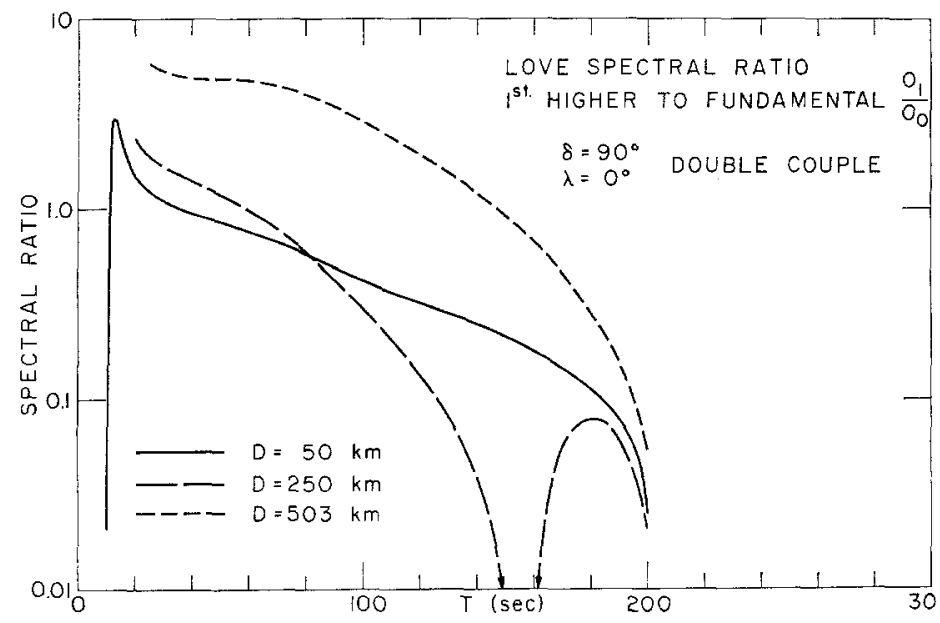

FIg. 10. Spectral ratio (Love first-mode/Love fundamental-mode) for a double couple at several values of depth in an oceanic model.

mum. On the other hand, at an azimuth of $30^{\circ}$, the spectral ratio has an easily identified minimum at $60 \mathrm{sec}$ for the $\left(75^{\circ}, 15^{\circ}\right)$ model. Another complicating factor, then, is that the minimums are sensitive to azimuth. It should also be remembered that these are spectral ratio minimums. Except for geometries where the minima are near zero, their presence may not be evident in either the Rayleigh or the Love spectra until the ratio has been formed.

The complications inherent in these spectral techniques can be demonstrated by 
considering the magnitude 5.2 Fallon earthquake of July 201962 (Toksöz et al., 1965). The fundamental Rayleigh and Love spectra measured at Ruth, Nevada, are shown in Figure 15. Taking their spectral ratio, $R_{z} / L$, we obtain a minimum near 26 sec which corresponds to focal depth of $20 \mathrm{~km}$ for a vertical strike-slip fault $\left(90^{\circ}, 0^{\circ}\right)$

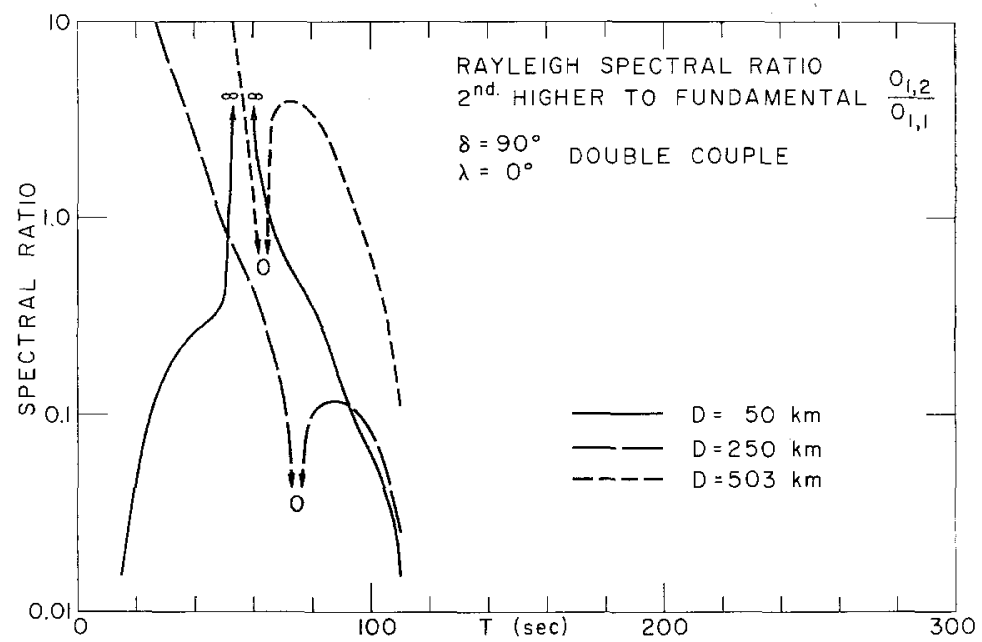

Fra. 11. Spectral ratio (Rayleigh second-mode/Rayleigh fundamental-mode) for a double couple at several values of depth in an oceanic model.

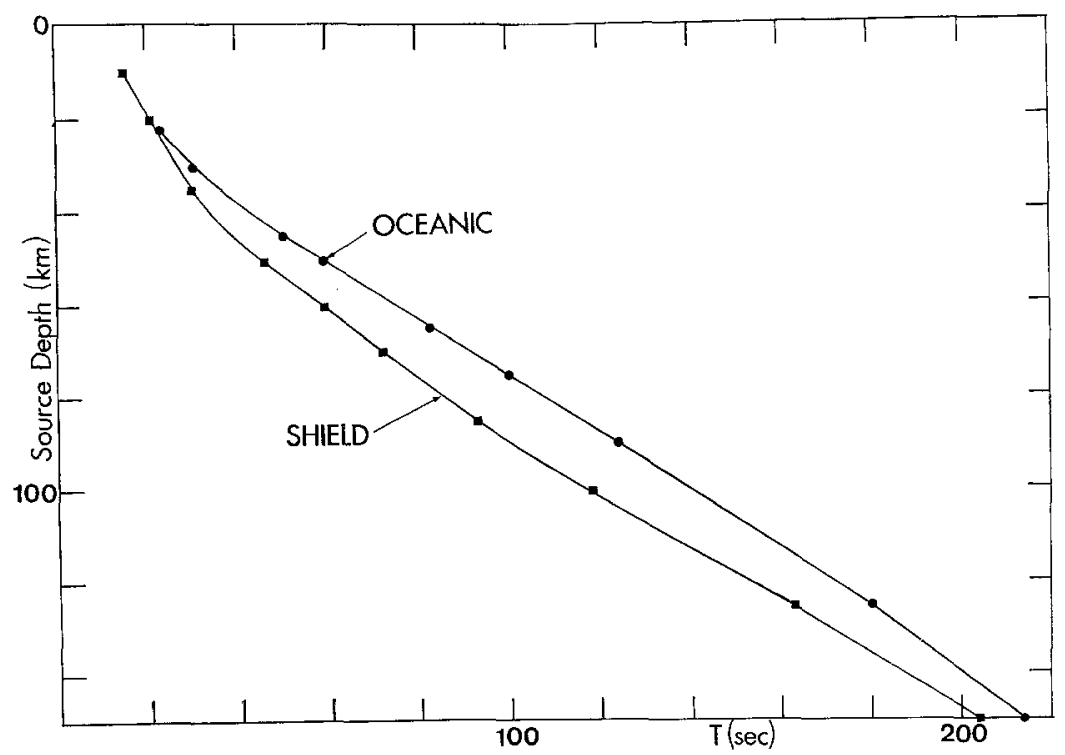

FIG. 12. Periods of spectral ratio minimums (Rayleigh fundamental-mode/Love fundamentalmode) versus source depth of a double couple in an oceanic and shield model.

and a Fallon-to-Ruth propagation path (Figure 16). The minimum is also apparent in the observed $R_{z}$.

The depth is the same as that obtained in Toksöz et al. (1965) by comparing the observed and theoretical spectra for the surface waves at Ruth, Pasadena and Jamestown. As in Tsai (1969), the assumed source-time variation was a step function. In order to fit the observed spectra at these stations, they required a fault orientation of 


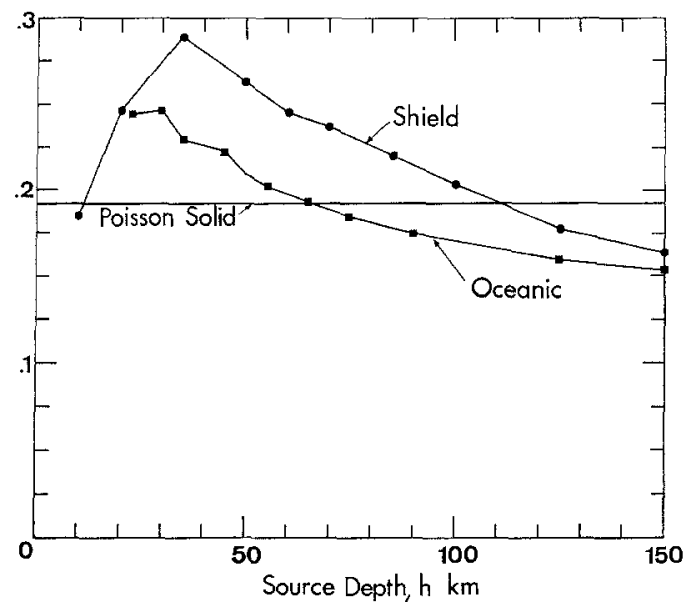

Fig. 13. Ratio of source depth to critical wavelength for the two earth models and a Poisson solid.

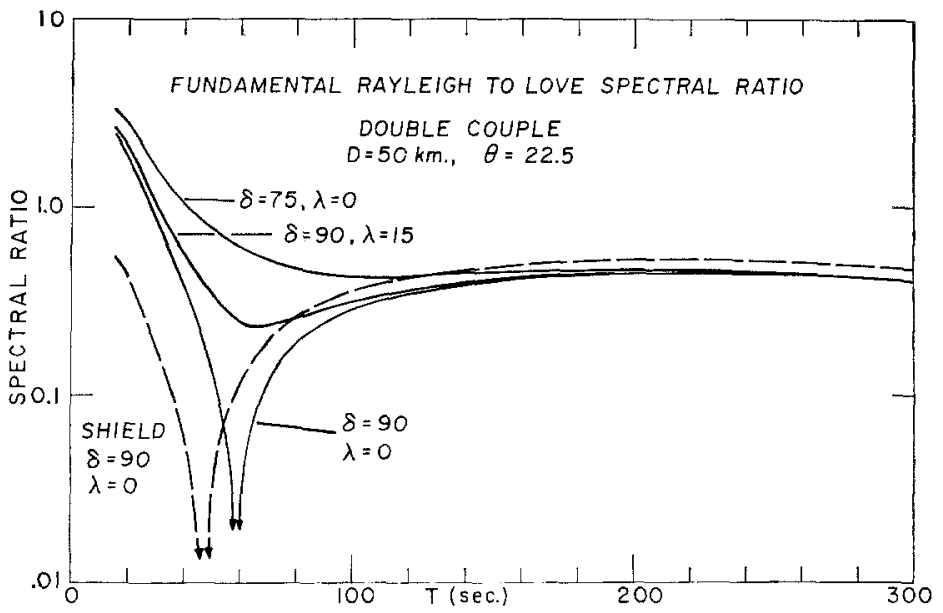

Frg. 14. Spectral ratio (Rayleigh fundamental-mode/Love fundamental-mode) for a double couple at selected fault orientations in an oceanic model.
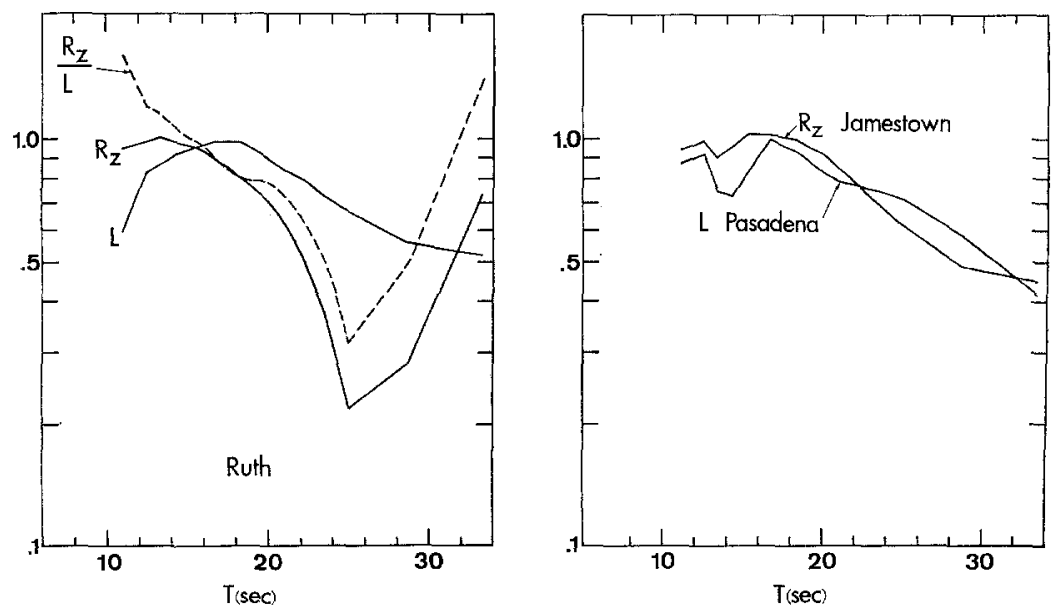

FIG. 15. Observed ground displacement spectra of Rayleigh and Love waves for the July 201962 Fallon earthquake. 
$\left(76^{\circ}, 230^{\circ}\right)$ and a strike azimuth of $355^{\circ}$. This corresponds to an azimuthal angle from: the strike of $\theta=100^{\circ}$ at Ruth. The $\left(90^{\circ}, 0^{\circ}\right)$ faults at a depth of 20 and $26 \mathrm{~km}$ require a $\theta=50^{\circ}-55^{\circ}$ or $\theta=125^{\circ}-130^{\circ}$ at Ruth in order to obtain a reasonable spectral ratio (Figure 16). This results in an unacceptable theoretical spectral ratio at Jamestown which is two orders of magnitude less than observed there. The spectral ratios for their $\left(76^{\circ}, 230^{\circ}\right)$ fault have barely perceptible minima only at $\theta$ near $40^{\circ}$ and $145^{\circ}$. There are no minima in $R_{z}$ at any azimuth for periods less than 40 sec.

The fault orientation was determined from a three-station fit of a radiation pattern of the Love- to Rayleigh-wave peak amplitudes ( $T=16 \mathrm{sec}$ ). Flinn, Lambert and Archambeau (1970), using the Fallon earthquake Rayleigh and Love waves recorded at 17 LRSM stations, found that the radiation pattern for the 16 -sec ratio could be fit. best by a $20-\mathrm{km}$-depth $\left(82^{\circ}, 196^{\circ}\right)$ fault plane with a strike azimuth of $10^{\circ}$. Since the: radiation pattern of the ratio at a given period is relatively insensitive to source depth, the $20-\mathrm{km}$ source depth was determined from the individual Love and Rayleigh radia-
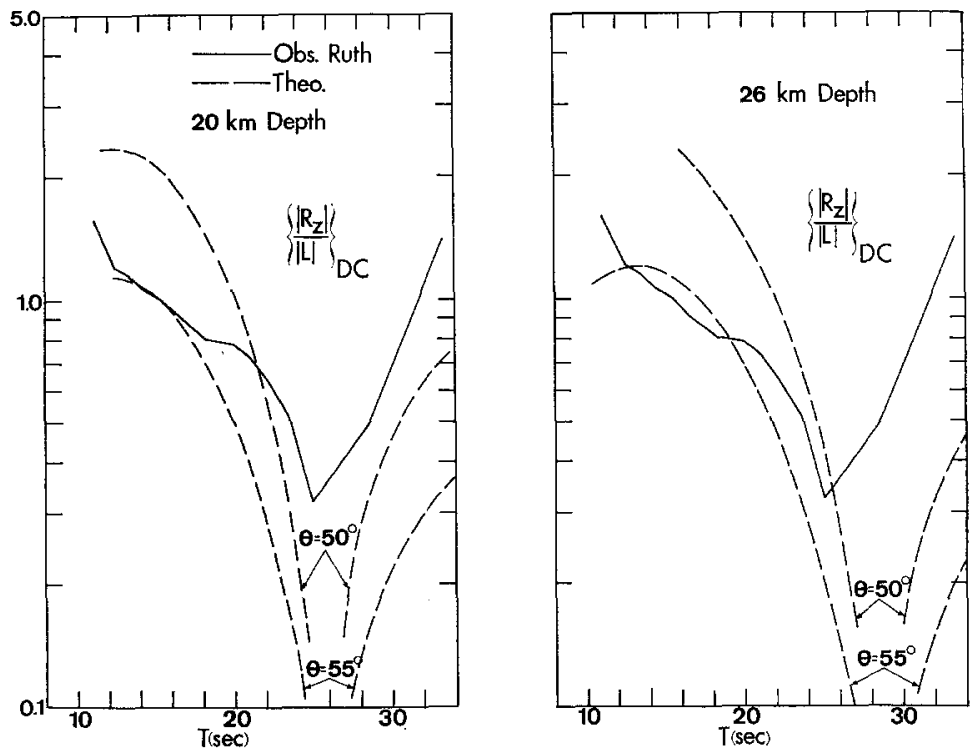

Fig. 16. Theoretical and observed spectral ratios at Ruth for two depths and azimuths.

tion patterns at various frequencies. The shapes of these individual patterns also admit a fault orientation of $\left(82^{\circ}, 174^{\circ}\right)$ (Flinn et al., 1970).

The spectral ratios at selected azimuths for the two LRSM determined fault solutions are shown in Figure 17 with the observed Ruth ratio. Again we cannot fit the Ruth data $\left(\theta=85^{\circ}\right)$ without violating the strike azimuth. The range of azimuth in which there is a detectable minimum in the $R_{z} / L$ ratio and in $R_{z}$ for a step function source are shown in Figure 18 for the LRSM fault solutions. In the same figure, we show the 16 -sec Love to Rayleigh radiation patterns.

Another piece of evidence that the fault strike azimuth is within $15^{\circ}$ of North as in Toksöz et al. (1965) and Flinn et al. (1970) can be found in the small dips in the absolute spectra of the Jamestown and Pasadena stations (Figure 15). Assuming that the Fallon event was due to a rupture moving to the North with a uniform velocity of 2.2 $\mathrm{km} / \mathrm{sec}$ along a fault segment of length $20 \mathrm{~km}$, these dips can be explained as the firsti minimums of the source propagation factor (Ben-Menahem, 1960). This factor would cause a minimum in the Rayleigh- and Love-wave spectra at just below the 10 -sec 
period at Ruth, at 14 sec at Pasadena, and at 12 to 14 sec at Jamestown. The minimum necessary length of $20 \mathrm{~km}$ is considered large for an earthquake of this magnitude (King and Knopoff, 1968). On the other hand, the shallowness of the minima can be explained by the rupture strength being much smaller at the ends of the fault relative to the center (Ben-Menahem and Toksöz, 1962). Thus the effective length contributing

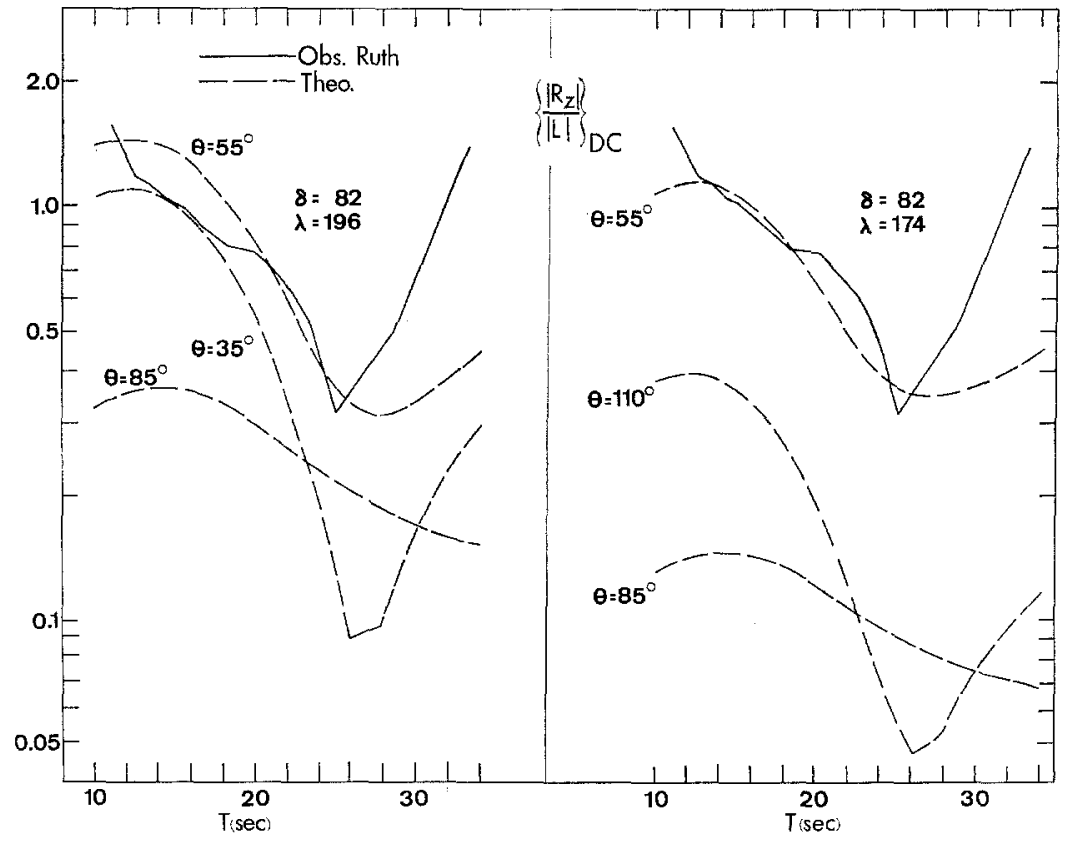

FIG. 17. Theoretical and observed spectral ratios at Ruth for two fault orientations and three azimuths for a $20-\mathrm{km}$ source depth.

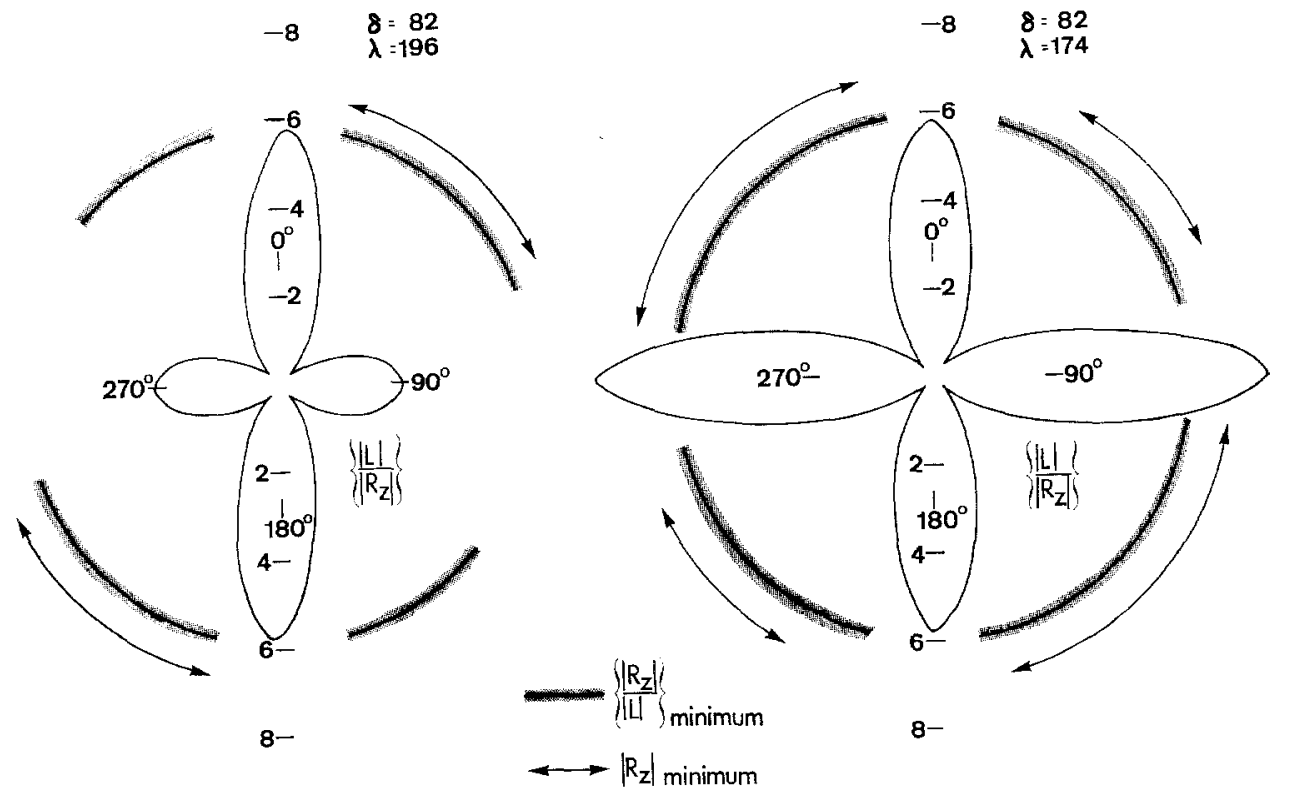

Frg. 18. Radiation patterns and ranges of azimuth with detectable spectral minimums for two LRSM determined fault solutions. 
most of the seismic energy could be much smaller and the spectra would still have the same minimal locations found in Figure 15.

Unless the solutions obtained from the three Caltech and the 17 LRSM stations are incorrect or can be shifted in strike azimuth a minimum of $25^{\circ}$ to the east, the dip in the Ruth, Nevada, Rayleigh spectrum and the Rayleigh to Love spectra ratio at 26 sec must be due to mechanisms other than source depth (Toksöz et al., 1965).

\section{CONCLUSIONS}

The tables of Rayleigh and Love displacement-stress or eigenvectors presented in this paper can be linearly combined for point sources or integrated for volume sources to calculate the spectra of surface waves. As an example of their use, spectral ratios were formed for various fault models in order to determine the feasibility of using spectral minimums or "holes" for source depth determinations.

For the minimum associated with the fundamental Rayleigh wave, the following conclusions can be made.

(1) The period for the spectral minimum is strongly dependent on fault slip, strike and station azimuth as well as source depth.

(2) For the vertical, pure strike-slip fault, a rough estimate of source depth in kilometers is given by the nodal periods in seconds.

(3) The fault orientation must be known from independent determinations in order to obtain the source depth.

The necessity of determining the fault orientation was demonstrated by the Fallon earthquake. If a vertical strike-slip fault had been assumed for this event, the minimum measured at Ruth, Nevada, would have corresponded to a depth equal to the USCGS reported depth. However, using fault solutions obtained from the spectra at other stations, it was found that the minimum was not related to the source depth.

Considering the difficulties and the sources of possible spectral contamination, it is hard to visualize spectral ratios playing an important role in the determination of focal mechanisms except for events where there is consistent and adequate station coverage, high signal-to-noise levels, and some supplementary information on the fault and propagation path.

\section{ACKNOWLEDGMEN'TS}

This research was supported by the Advanced Research Projects Agency and was monitored by the Air Force Office of Scientific Research under contract AF-F44620-68-C-0082.

\section{APPENDIX I}

COMPOUND MATRIX ELEMENTS

Expressions for the compound layer matrix are

$$
\begin{aligned}
a_{11}=a_{66}= & -2 \gamma(\gamma-1)+\left(2 \gamma^{2}-2 \gamma+1\right) C P \cdot C Q-\gamma^{2} S T P \cdot S T Q \\
& \quad-\left(\gamma^{2}-1\right) S D P \cdot S D Q \\
a_{12}=a_{56}= & i\left(\rho c^{2}\right)^{-1}[C P \cdot S D Q+C Q \cdot S T P] \\
a_{13}=a_{14}=a_{36}= & -\left(\rho c^{2}\right)^{-1}[(2 \gamma-1)(1-C P \cdot C Q)+(\gamma-1) S D P \cdot S D Q \\
& \quad+\gamma S T P \cdot S T Q] \\
= & a_{46}
\end{aligned}
$$




$$
\begin{aligned}
a_{15}=a_{26}= & -i\left(\rho c^{2}\right)^{-1}[C Q \cdot S D P+C P \cdot S T Q] \\
a_{16}= & \left(\rho c^{2}\right)^{-2}[2(1-C P \cdot C Q)+S T P \cdot S T Q+S D P \cdot S D Q] \\
a_{21}=a_{65}= & i\left(\rho c^{2}\right)\left[(\gamma-1)^{2} C Q S D P+\gamma^{2} C P S T Q\right] \\
a_{22}=a_{55}= & C P \cdot C Q \\
a_{23}=a_{24}=a_{35}=a_{45}= & i[(\gamma-1) C Q \cdot S D P+\gamma C P \cdot S T Q] \\
a_{25}= & S D P \cdot S T Q \\
a_{31}=a_{41}=a_{63}= & \left(\rho c^{2}\right)\left[\gamma(\gamma-1)(2 \gamma-1)(1-C P \cdot C Q)+(\gamma-1)^{3} S D P \cdot S D Q\right. \\
= & \quad a_{64} \\
a_{32}=a_{42}= & -i[(\gamma-1) C P \cdot S D Q+\gamma C Q \cdot S T P] \\
= & a_{53}=a_{54} \\
a_{33}=a_{44}= & 1+2 \gamma(\gamma-S T Q] \\
& +\gamma^{2} S T P S T Q \\
a_{33}-1= & a_{43} \\
a_{51}=a_{62}= & -i\left(\rho c^{2}\right)\left[(\gamma-1)^{2} C P \cdot S D Q+\gamma^{2} C Q S T P\right] \\
a_{52}= & S T P \cdot S D Q \\
a_{34}= & \left(\rho c^{2}\right)^{2}\left[2 \gamma^{2}(\gamma-1)^{2}(1-C P \cdot C Q)+(\gamma-1)^{4} S D P \cdot S D Q\right. \\
& \left.+\gamma^{4} S T P \cdot S T Q\right]
\end{aligned}
$$

where

$$
\begin{aligned}
C P & =\cos \left(k r_{\alpha} d\right), \quad C Q=\cos \left(k r_{\beta} d\right) \\
S T P & =r_{\alpha} \sin \left(k r_{\alpha} d\right), \quad S T Q=r_{\beta} \sin \left(k r_{\beta} d\right) \\
S D P & =\frac{\sin \left(k r_{\alpha} d\right)}{r_{\alpha}} S D Q=\frac{\sin \left(k r_{\beta} d\right)}{r_{\beta}} \\
\gamma & =2\left(\frac{\beta}{c}\right)^{2}
\end{aligned}
$$

and the layer $m$ subscript has been suppressed in the notation.

Combining the product matrix with its inverse as given in Harkrider (1964), we have the following identities for the compound product matrix, $a$.

$$
\begin{array}{rlrl}
a_{13} & \equiv a_{14} & a_{31} & \equiv a_{41} \\
a_{23} & \equiv a_{24} & a_{32} & \equiv a_{42} \\
a_{33} & \equiv a_{34}+1 & a_{33} & \equiv a_{43}+1 \\
a_{43} & \equiv a_{44}-1 & a_{34} & \equiv a_{44}-1 \\
a_{53} & \equiv a_{54} & a_{35} & \equiv a_{45} \\
a_{63} & \equiv a_{64} & a_{36} & \equiv a_{46} \\
& & a_{33} \equiv a_{44} & \\
a_{34} \equiv a_{43} &
\end{array}
$$


and from the definition.

$$
\begin{gathered}
a_{26} \equiv a_{15}, \quad a_{35} \equiv a_{23}, \quad a_{36} \equiv a_{13}, \quad a_{53} \equiv a_{32}, \quad a_{55} \equiv a_{22}, \quad a_{56} \equiv a_{12}, \\
a_{62} \equiv a_{51}, \quad a_{63} \equiv a_{31}, \quad a_{65} \equiv a_{21}, \quad \text { and } a_{66} \equiv a_{11} \\
\text { APPENDIX II } \\
\text { LONG-PERIOD LIMITS }
\end{gathered}
$$

Using the limits of the Thomson-Haskell matrices at long periods, we obtain the following Love-wave limits for $c>0$ as $\omega$ and $k \rightarrow 0$

$$
\begin{gathered}
F_{L} \equiv-\left(A_{L}\right)_{21}^{*}-\mu_{n} r_{\beta n}^{*}\left(A_{L}\right)_{11}=0 \\
F_{L} \rightarrow-k \sum_{j=1}^{n-1} \mu_{j} r_{\beta j}^{2} d_{j}-\mu_{n} r_{\beta n}^{*} \rightarrow \mu_{n}\left(1-\frac{c^{2}}{\beta_{n}^{2}}\right)^{1 / 2}
\end{gathered}
$$

thus

$$
c \rightarrow \boldsymbol{\beta}_{n}
$$

$$
\begin{aligned}
& \frac{\partial F_{L}}{\partial k} \rightarrow-\sum_{j=1}^{n-1} \mu_{j}\left(1+\frac{c^{2}}{\beta_{j}{ }^{2}}\right) d_{j}-\mu_{n} \frac{c^{2}}{{\beta_{n}}^{2}} \frac{1}{k r_{\beta n}^{*}} \\
& \frac{\partial F_{L}}{\partial \omega} \rightarrow-2 \sum_{j=1}^{n-1} c \rho_{j} d_{j}+\mu_{n} \frac{c}{\beta_{n}{ }^{2}} \frac{1}{k r_{\beta_{n}}^{*}}
\end{aligned}
$$

thus

$$
U=-\left(\frac{\partial F_{L}}{\partial k}\right) /\left(\frac{\partial F_{L}}{\partial \omega}\right) \rightarrow c \rightarrow \beta_{n}
$$

and

$$
\begin{aligned}
\underline{A}_{L} & =\frac{1}{\left(A_{L}\right)_{11} \frac{\partial F_{L}}{\partial k}} \rightarrow-k \frac{r_{\beta n}^{*}}{\mu_{n}} \\
& \rightarrow-\frac{\mu_{p}}{\mu_{n}{ }^{2}} h_{p}\left(1-\frac{\beta_{n}{ }^{2}}{\beta_{p}{ }^{2}}\right) k^{2} \\
& \rightarrow O\left(k^{2}\right)
\end{aligned}
$$

since $F_{L}=0$ implies from above that

$$
\mu_{n} r_{\beta n}^{*} \rightarrow-k \sum_{j=1}^{n-1} \mu_{j} r_{\beta j}^{2} d_{j}=-k h_{p} \mu_{p}\left(\frac{c^{2}}{\beta_{p}^{2}}-1\right)
$$

where

$$
h_{p} \equiv \sum_{j=1}^{n-1} h_{j}, \beta_{p}^{2} \equiv \frac{\mu_{p}}{\rho_{p}}, \mu_{p} \equiv \sum_{j=1}^{n-1} \mu_{j} d_{j} / h_{p}
$$

and

$$
\rho_{p} \equiv \sum_{j=1}^{n-1} \rho_{j} d_{j} / h_{p}
$$


The summation over $j$ is from layer 1 to layer $n-1$, with $n$ denoting the solid halfspace.

The $p$ subscript was used above because for Love waves in a multilayered free plate

$$
F_{L} \equiv\left(A_{L}\right)_{21}^{*}=0
$$

and at long periods

$$
\left(A_{L}\right)_{21}^{*} \rightarrow k \sum_{j=1}^{n-1} \mu_{j} r_{\beta_{j}}^{2} d_{j} \rightarrow-k h_{p} \mu_{p}\left(1-\frac{c^{2}}{\beta_{p}^{2}}\right)
$$

thus $F_{L}=0$ can be satisfied by $c=\beta_{p}$ for small $k$.

For Rayleigh waves for an all solid model as $\omega$ and $k \rightarrow 0$

$$
\begin{gathered}
F_{R}=N K+L^{*} M^{*}=0 \\
F_{R} \rightarrow-\left[\left(\gamma_{n}-1\right)^{2}+\gamma_{n}^{2} r_{\alpha n} r_{\beta n}\right] \\
\frac{\dot{u}_{0}^{*}}{\dot{w}_{0}}=\frac{K}{L^{*}} \rightarrow \frac{\left(\gamma_{n}-1\right)}{\gamma_{n} r_{\alpha n}^{*}} \\
G N^{*}-L^{*} H \rightarrow-\frac{r_{\alpha n}^{*}}{\rho_{n} c^{2}}
\end{gathered}
$$

where

$$
\gamma_{n}=2\left(\frac{\beta_{n}}{c}\right)^{2}
$$

thus $F_{R}=0$ implies $c \rightarrow V_{R n}$ : the root of Rayleigh's equation (4),

$$
\begin{aligned}
& \frac{\partial F_{R}}{\partial k} \rightarrow \frac{4}{k}\left\{\left(\gamma_{n}-1\right)+\frac{\beta_{n}{ }^{2} \gamma_{n}{ }^{2}}{C^{2} \alpha_{n}{ }^{2}} \frac{\left[2 C^{2}-\alpha_{n}{ }^{2}-\beta_{n}{ }^{2}\right]}{\left(\gamma_{n}-1\right)^{2}}\right\} \\
& \frac{\partial F_{R}}{\partial \omega} \rightarrow \frac{1}{C} \frac{\partial F_{R}}{\partial k}
\end{aligned}
$$

thus

$$
U=-\left(\frac{\partial F_{R}}{\partial k}\right) /\left(\frac{\partial F_{R}}{\partial \omega}\right) \rightarrow C \rightarrow V_{R n}
$$

and

$$
\underline{A}_{R}=\frac{\left[G^{*} N-L^{*} H\right]}{\left(\frac{\partial F_{R}}{\partial k}\right)}
$$

thus

$$
\begin{aligned}
\underline{A}_{R} & \rightarrow \frac{r_{\alpha n}^{*}}{4 \rho_{n} V_{R n}^{2}\left\{\left(\gamma_{n}-1\right)+\frac{\beta_{n}^{2}}{V_{R n}^{2}} \frac{\gamma_{n}^{2}}{\alpha_{n}{ }^{2}} \frac{\left[2 V_{R n}^{2}-\alpha_{n}{ }^{2}-\beta_{n}^{2}\right]}{\left(\gamma_{n}-1\right)^{2}}\right\}} \cdot k \\
& \rightarrow O(k) .
\end{aligned}
$$


For Rayleigh waves in a multilayered free plate

$$
\begin{aligned}
& F_{R}=\left[A_{32}^{*} A_{41}^{*}+A_{31} A_{42}\right] \\
& F_{R} \rightarrow k^{2} C^{4} \sum_{j=1}^{n-1} \rho_{j} d_{j} \cdot \sum_{j=1}^{n-1} \rho_{j}\left[1-2 \gamma_{j}\left(1-\frac{\beta_{j}{ }^{2}}{\alpha_{j}{ }^{2}}\right)\right] d_{j}
\end{aligned}
$$

retaining first order terms as $k \rightarrow 0$, thus $F_{R}=0$ can be satisfied by a $c$ such that

$$
\sum_{j=1}^{n-1} \rho_{j}\left[1-2 \gamma_{j}\left(1-\frac{\beta_{j}^{2}}{\alpha_{j}^{2}}\right)\right] d_{j}=0
$$

for small $k$; i.e.

$$
c^{2}=\frac{\sum_{j=1}^{n-1} \rho_{j} V_{p j}^{2} d_{j}}{\sum_{j=1}^{n-1} \rho_{j} d_{j}}
$$

where

$$
V_{p j}^{2}=4 \beta_{j}{ }^{2}\left(1-\frac{\beta_{j}{ }^{2}}{\alpha_{j}^{2}}\right)
$$

APPENDIX III

\section{SHORT-PERIOD LIMITS}

At short periods, the relations for Love waves in a multilayered half-space reduce to those for Love waves in the top layer over a half-space with second layer properties.

Thus as $k \rightarrow \infty$

$$
F_{L} \rightarrow-\mu_{1} r_{\beta 1} \sin Q_{1}-\mu_{2} r_{\beta 2}^{*} \cos Q_{1}=0
$$

and as $c \rightarrow \beta_{1}$

$$
\begin{aligned}
& k d_{1} \rightarrow \frac{(2 m+1)}{2} \pi\left[\left(c / \beta_{1}\right)^{2}-1\right]^{-1 / 2} \rightarrow \infty \\
& \frac{\partial F_{L}}{\partial k} \rightarrow\left[\mu_{1} d_{1}+\mu_{2}\left(1-\frac{\beta_{1}{ }^{2}}{\beta_{2}{ }^{2}}\right)^{1 / 2} k d_{1}^{2}\right] \frac{2}{(2 m+1) \pi} \\
& \frac{\partial F_{L}}{\partial \omega} \rightarrow-\left[\mu_{1} \frac{d_{1}}{\beta_{1}}+\mu_{2}\left(1-\frac{\beta_{1}^{2}}{\beta_{2}{ }^{2}}\right)^{1 / 2} \frac{k d_{1}^{2}}{\beta_{1}}\right] \frac{2}{(2 m+1) \pi}
\end{aligned}
$$

thus

$$
U=-\left(\frac{\partial F_{L}}{\partial \hbar}\right) /\left(\frac{\partial F_{L}}{\partial \omega}\right) \rightarrow \beta_{1}
$$


and

$$
\underline{A}_{L} \rightarrow \frac{1}{\mu_{1} d_{1}}
$$

For Rayleigh waves, the multilayered solid half-space reduces to a half-space of the top-layer properties and the short-period limits are the same as the long-period limits in Appendix II with the subscript 1 replacing the $n$.

\section{References}

Aki, K. (1967). Scaling law of seismic spectrum, J. Geophys. Res. 72, 1217-1231.

Ben-Menahem, A. (1961). Radiation of seismic surface-waves from finite moving sources, Bull. Seism. Soc. Am. 51, 401-435.

Ben-Menahem, A. and D. G. Harkrider (1964). Radiation patterns of seismie surface waves from buried dipolar point sources in a flat stratified earth, J. Geophys. Res. 69, 2605-2620.

Ben-Menahem, A. and M. N. Toksöz (1962). Source mechanism from spectra of long-period seismic waves, 1. The Mongolian earthquake of December 4, 1957, J. Geophys. Res. 67, 1943-1955.

Ben-Menahem, A. and M. N. Toksöz (1963). Source mechanism from spectrums of long-period surface waves, 2. The Kamchatka earthquake of November 4, 1952, J. Geophys. Res. 68, 52075222 .

Dunkin, J. W. (1965). Computation of modal solutions in layered, elastic media at high frequencies, Bull. Seism. Soc. Am. 55, 335-358.

Flinn, E. A., D. G. Lambert, and C. B. Archambeau (1970). Detection, analysis, and interpretation of teleseismic signals, 2. Love and Rayleigh waves from Bilby, Shoal, and Fallon, (unpublished data).

Gilbert, F. and G. E. Backus (1966). Propagator matrices in elastic wave and vibration problems, Geophysics 31, 326-332.

Harkrider, D. G. (1964). Surface waves in multilayered elastic media, 1. Rayleigh and Love waves from buried sources in a multilayered elastic half-space, Bull. Seism. Soc. Am. 54, 627-679.

Harkrider, D. G. and D. L. Anderson (1962). Computation of surface-wave dispersion for multilayered anisotropic media, Bull. Seism. Soc. Am. 52, 321-332.

Harkrider, D. G. and D. L. Anderson (1966). Surface wave energy from point sources in plane layered Earth models, J. Geophys. Res. 71, 2967-2980.

Haskell, N. A. (1953). Dispersion of surface waves on multilayered media, Bull. Seism. Soc. Am.43, $17-34$.

Haskell, N. A. (1963). Radiation pattern of Rayleigh waves from a fault of arbitrary dip and direction of motion in a homogeneous medium, Bull. Seism. Soc. Am. 53, 619-642.

Haskell, N. A. (1964). Total energy and energy spectral density of elastic wave radiation from propagating faults, Bull. Seism. Soc. Am. 54, 1811-1842.

Haskell, N. A. (1966). Total energy and energy spectral density of elastic wave radiation from propagating faults, Part II. A statistical source model, Bull. Seism. Soc. Am. 56, 125-140.

Keilis-Borok, V. I. and T. B. Yanovskaya (1962). Dependence of the spectrum of surface waves on the depth of the focus within the Earth's crust, Bull. Acad. Sci., USSR., Geophys Ser., English Transl. 11, 1532-1539.

King, D. H. and L. Knopoff (1968). Stress drop in earthquakes, Bull. Seism. Soc. Am. 58, 249-257.

Knopoff, L. and A. F. Gangi (1959). Seismic reciprocity, Geophysics 24, 681-691.

Press, F., D. G. Harkrider, and C. A. Seafeldt (1961). A fast, convenient program for computation of surface-wave dispersion curves in multilayered media, Bull. Seism. Soc. Am. 5l, 495-502.

Rosenbaum, J. H. (1964). A note on the computation of Rayleigh-wave dispersion curves for layered media, Bull. Seism. Soc. Am. 54, 1013-1019.

Saito, M. (1967). Excitation of free oscillations and surface waves by a point source in a vertically heterogeneous Earth, J. Geophys. Res. 72, 3689-3700.

Thrower, E. N. (1965). The computation of dispersion curves on layered media, J. Sound Vib. 2, $3,14-30$.

Toksöz, M. N., D. G. Harkrider, and A. Ben-Menahem (1965). Determination of source parameters by amplitude equilization, 2 . Release of tectonic strain by underground nuclear explosions and mechanisms of earthquakes, $J$. Geophys. Res. 70, 907-922. 
Tsai, Yi-Ben (1969). Determination of focal depths of earthquakes in the mid-ocean ridges from amplitude spectra of surface waves, Thesis for Ph.D., Massachusetts Institute of Technology.

Vilkovitch, E. V., A. L. Levshin, and M. G. Neigaus (1966). Love waves in a vertically inhomogeneous medium, machine interpretation of seismic waves, Electronic Computer Seismology, Part 2, 130-149.

Vlaar, N. J. (1966). The field from an $S H$ point source in a continuously layered homogeneous half-space. Part II. The field in a half-space, Bull. Seism. Soc. Am. 56, 1305-1316.

Department of Geological Sciences

BROWN UNIVERSITY

Providence, Rhode Island 02912

Contribution No.

Manuscript received April 141970 